SLAC-PUB-6497

RU-94-67

hep-ph/9408359

August 1994

$\mathrm{T} / \mathrm{E}$

\title{
Determination of Fundamental Supersymmetry Parameters from Chargino Production at LEP II
}

\author{
Jonathan L. Feng 因团 \\ Stanford Linear Accelerator Center \\ Stanford University, Stanford, California 94309 \\ Matthew J. Strassler \\ Department of Physics and Astronomy \\ Rutgers University, Piscataway, New Jersey 08855
}

\begin{abstract}
If accessible at LEP II, chargino production is likely to be one of the few available supersymmetric signals for many years. We consider the prospects for the determination of fundamental supersymmetry parameters in such a scenario. The study is complicated by the dependence of observables on a large number of these parameters. We propose a straightforward procedure for disentangling these dependences and demonstrate its effectiveness by presenting a number of case studies at representative points in parameter space. Working in the context of the minimal supersymmetric standard model, we find that chargino production by itself is a fairly sensitive probe of the supersymmetry-breaking sector. For significant regions of parameter space, it is possible to test the gaugino mass unification hypothesis and to measure the gaugino contents of the charginos and neutralinos, thereby testing the predictions of grand unification and the viability of the lightest supersymmetric particle as a dark matter candidate. For much of the parameter space, it is also possible to set limits on the mass of the electron sneutrino, which provide a valuable guide for future particle searches.
\end{abstract}

(Submitted to Physical Review D)

*Work supported by the Department of Energy, contract DE-AC03-76SF00515.

${ }^{\dagger}$ Work supported in part by an NSF Graduate Research Fellowship.

${ }^{\ddagger}$ Work supported in part by the Department of Energy, contract DE-FG05-90ER40559. 


\section{INTRODUCTION}

The gauge hierarchy problem has motivated many approaches to extending the standard model, and among them, supersymmetry (SUSY) is one of the most promising [1]. If SUSY is to provide a solution to this problem, it must be broken at energies of order $1 \mathrm{TeV}$, and so the masses of supersymmetric particles must lie at or below this energy. Because such energies are within reach of existing accelerators or those that are scheduled to operate in the near future, SUSY phenomenology has attracted much attention in recent years.

In many of the supersymmetric models that have been explored, charginos are the lightest observable supersymmetric particles, and for this reason, chargino searches have been particularly well-studied [2, 3, $4,5,6,6,7,8,9,9,10$. The current lower bound on the mass of the lighter chargino is $45 \mathrm{GeV}$ [11,12]. Chargino discovery studies have shown that, with the characteristics currently expected to be reached at LEP II, $\sqrt{s}=175-190 \mathrm{GeV}$ and a luminosity of 200-500 $\mathrm{pb}^{-1} /$ year/experiment [13], the discovery reach will extend nearly to the kinematic limit, significantly extending the accessible region of parameter space. It is also worth noting that there are at present tentative but tantalizing hints of the possible existence of light charginos from the measurement of $A_{L R}$ at SLC [14,15] and measurements of $\Gamma(Z \rightarrow b \bar{b})$ at LEP and SLC [16, 17, 18, 19].

If charginos are discovered at LEP II, they will provide one of the first direct signals of supersymmetry and may well prove to be the most promising candidates for precision supersymmetry studies for many years. Although neutralino production is likely to accompany chargino production, it often suffers from a significantly smaller cross section [20 and may be more difficult to separate from backgrounds [6.7]. It is therefore natural to ask what information about the parameters of supersymmetry can be obtained from the chargino signal alone.

In the case of linear $e^{+} e^{-}$colliders with $\sqrt{s}=500 \mathrm{GeV}$, the question of precision measurements of sparticle masses and underlying SUSY parameters has been addressed in a number of studies [21,22, 23, 24, 25, 26, 27,28]. These studies have shown that if a number of sparticles are light enough to be produced, their masses can be determined to high accuracy, and the sparticle spectrum can provide stringent tests of standard theoretical assumptions. If light charginos exist, thousands of them will be produced at LEP II, and precision measurements might also be possible there. However, in addition to the difficulties present in all studies of SUSY signals, such as unobservable particles in the final state and a wealth of unknown parameters, chargino production at LEP II suffers from other difficulties not present in the $500 \mathrm{GeV}$ collider studies. In particular, the background from $W$ pair production will have a stronger overlap with the chargino signal, and beam polarization, an important diagnostic in linear collider experiments, will not be available. The aim of this paper is to determine to what extent these difficulties can be overcome, and to explore the prospects for the determination of fundamental SUSY parameters in different regions of parameter space.

A previous study [29] addressed this question with the assumptions that charginos are lighter than $W$ bosons, that the vacuum expectations values of the two Higgs fields are roughly equal, and, for most of the analysis, that sneutrinos are either very heavy or very light. We will relax these assumptions so that we may determine to what extent they may be tested. Our study will be conducted in the context of the minimal supersymmetric standard model (MSSM) without gaugino mass unification. We will find that for some regions of 
parameter space, it is possible to test gaugino mass unification, a general prediction of supersymmetric grand unified theories, and also to place strong bounds on the mass of the electron sneutrino, which would provide a valuable guide for designing future sparticle searches. In addition, we will see that it is often possible to measure the gaugino contents of the charginos and neutralinos, which have implications for the viability of the lightest neutralino as a dark matter candidate [30].

Formally, every observable defines a hypersurface in the space of SUSY parameters, and determining the parameters simply consists of finding the intersections of these hyperplanes. Practically, there are many possible observables with varying degrees of dependence on the fundamental parameters and different experimental uncertainties, and reducing the allowed volume to a small region is at first sight far from straightforward. The most effective way to extract the underlying parameters from the data is to perform a binned maximum likelihood fit, and ultimately, this is what should be done. However, such a procedure does not provide much physical understanding of the results, nor does it provide a useful way to visualize how the underlying parameters are constrained by specific measurements. In this study, we will discuss observables one by one in a way that gives a straightforward strategy for disentangling their complicated dependences. In the process we will show which parameters can be tightly constrained by chargino production and which cannot. We hope that this study will provide some general understanding of the results one may hope to achieve. Of course, if light charginos are found, this picture will be considerably sharpened by strategies tailored to the particular point in parameter space that is realized in nature.

In Sec. II we briefly review the MSSM. We state our assumptions about the MSSM and discuss the theoretical prejudices that we hope to test through our approach. We then describe the region of parameter space in which charginos can be produced at LEP II and present the SUSY parameter space that we hope to constrain. In Sec. III] we describe the salient aspects of chargino events and discuss the observables that will be most central to our analysis. Sec. $\mathbb{\nabla}$ contains a description of the event simulation and the cuts used in the case studies. The case studies themselves are presented in Sec. $\mathrm{V}$, where we describe the strategy that we will follow in systematically constraining the SUSY parameters and then apply it to a number of representative points in parameter space. We conclude with some final comments and a summary of our results in Sec. VI.

\section{SUSY PARAMETER SPACE AND CHARGINO PRODUCTION}

\section{A. The Minimal Supersymmetric Standard Model}

Our analysis will be performed in the context of the MSSM [1, 31], the simplest extension of the standard model that includes supersymmetry. In this subsection, we explain which assumptions about the MSSM we will make, and we introduce the SUSY parameters that we hope to constrain.

The MSSM includes the usual matter superfields and two Higgs doublet superfields

$$
\hat{H}_{1}=\left(\begin{array}{c}
\hat{H}_{1}^{0} \\
\hat{H}_{1}^{-}
\end{array}\right) \quad \text { and } \quad \hat{H}_{2}=\left(\begin{array}{c}
\hat{H}_{2}^{+} \\
\hat{H}_{2}^{0}
\end{array}\right) \text {, }
$$


where $\hat{H}_{1}$ and $\hat{H}_{2}$ give masses to the isospin $-\frac{1}{2}$ and $+\frac{1}{2}$ fields, respectively. These two superfields are coupled in the superpotential through the term $-\mu \epsilon_{i j} \hat{H}_{1}^{i} \hat{H}_{2}^{j}$, where $\mu$ is the supersymmetric Higgs mass parameter. The ratio of the two Higgs scalar vacuum expectation values is defined to be $\tan \beta \equiv\left\langle H_{2}^{0}\right\rangle /\left\langle H_{1}^{0}\right\rangle$. Soft supersymmetry-breaking terms [32] for scalars and gauginos are included in the MSSM with

$$
V_{\text {soft }}=\sum_{i} m_{i}^{2}\left|\phi_{i}\right|^{2}+\frac{1}{2}\left\{\left[M_{1} \tilde{B} \tilde{B}+\sum_{j=1}^{3} M_{2} \tilde{W}^{j} \tilde{W}^{j}+\sum_{k=1}^{8} M_{3} \tilde{g}^{k} \tilde{g}^{k}\right]+\text { h.c. }\right\}+[A \text { terms }],
$$

where $i$ runs over all scalar multiplets. The " $A$ terms" are cubic scalar terms that couple the Higgs scalars to squarks and to sleptons with coefficients $A_{u_{i}} A_{d_{i}}$, and $A_{e_{i}}$, where $i$ is the generation number. Studies of the MSSM often assume a number of relations among the mass parameters $m_{i}$ and $M_{i}$; the relatively weak assumptions made in this study will be detailed shortly.

The charginos and neutralinos of the MSSM are the mass eigenstates that result from the mixing of the electroweak gauginos $\tilde{B}$ and $\tilde{W}^{j}$ with the Higgsinos. The charged mass terms that appear are

$$
\left(\psi^{-}\right)^{T} \mathbf{M}_{\tilde{\chi}^{ \pm}} \psi^{+}+\text {h.c. }
$$

where $\left(\psi^{ \pm}\right)^{T}=\left(-i \tilde{W}^{ \pm}, \tilde{H}^{ \pm}\right)$and

$$
\mathbf{M}_{\tilde{\chi}^{ \pm}}=\left(\begin{array}{cc}
M_{2} & \sqrt{2} M_{W} \sin \beta \\
\sqrt{2} M_{W} \cos \beta & \mu
\end{array}\right) .
$$

The chargino mass eigenstates are $\tilde{\chi}_{i}^{+}=\mathbf{V}_{i j} \psi_{j}^{+}$and $\tilde{\chi}_{i}^{-}=\mathbf{U}_{i j} \psi_{j}^{-}$, where the unitary matrices $\mathbf{U}$ and $\mathbf{V}$ are chosen to diagonalize $\mathbf{M}_{\tilde{\chi}^{ \pm}}$. Neutral mass terms may be written as

$$
\frac{1}{2}\left(\psi^{0}\right)^{T} \mathbf{M}_{\tilde{\chi}^{0}} \psi^{0}+\text { h.c. },
$$

where $\left(\psi^{0}\right)^{T}=\left(-i \tilde{B},-i \tilde{W}^{3}, \tilde{H}_{1}^{0}, \tilde{H}_{2}^{0}\right)$ and

$$
\mathbf{M}_{\tilde{\chi}^{0}}=\left(\begin{array}{cccc}
M_{1} & 0 & -M_{Z} \cos \beta \sin \theta_{W} & M_{Z} \sin \beta \sin \theta_{W} \\
0 & M_{2} & M_{Z} \cos \beta \cos \theta_{W} & -M_{Z} \sin \beta \cos \theta_{W} \\
-M_{Z} \cos \beta \sin \theta_{W} & M_{Z} \cos \beta \cos \theta_{W} & 0 & -\mu \\
M_{Z} \sin \beta \sin \theta_{W} & -M_{Z} \sin \beta \cos \theta_{W} & -\mu & 0
\end{array}\right) .
$$

The neutralino mass eigenstates are $\tilde{\chi}_{i}^{0}=\mathbf{N}_{i j} \psi_{j}^{0}$, where $\mathbf{N}$ diagonalizes $\mathbf{M}_{\tilde{\chi}^{0}}$. In order of increasing mass the four neutralinos are labeled $\tilde{\chi}_{1}^{0}, \tilde{\chi}_{2}^{0}, \tilde{\chi}_{3}^{0}$, and $\tilde{\chi}_{4}^{0}$, and the two charginos, similarly ordered, are $\tilde{\chi}_{1}^{ \pm}$and $\tilde{\chi}_{2}^{ \pm}$. From the mass matrices in Eqs. (幽) and (6), it can be seen that in the limits $\tan \beta \rightarrow 0$ and $\tan \beta \rightarrow \infty$ there is an exact symmetry $\mu \leftrightarrow-\mu$.

In the form outlined above, the MSSM contains many unknown parameters, and typically a number of simplifying assumptions are made. These assumptions are usually based on grand unified theories or minimal supergravity. As we hope to test such theories, we choose less restrictive and more phenomenological assumptions, which we list below. Having stated our assumptions, we will then explain our reasons for choosing them and explore their implications. 


\section{B. Our Basic Assumptions}

In this study, we will make the following assumptions:

(a) R-parity is conserved.

(b) The lightest supersymmetric particle (LSP) is the lightest neutralino, $\tilde{\chi}_{1}^{0}$.

(c) Sleptons and squarks have masses beyond the kinematic limit of LEP II.

(d) The gluino is heavier than the lighter chargino.

(e) The intergenerational mixing in the squark, slepton, and quark sectors is small and may be neglected in our analysis.

(f) The four left-handed squarks of the first two generations are nearly degenerate at low energy with mass $m_{\tilde{q}}$, as are all six left-handed sleptons with mass $m_{\tilde{l}}$ :

$$
\begin{aligned}
& m_{\tilde{u}_{L}} \approx m_{\tilde{d}_{L}} \approx m_{\tilde{c}_{L}} \approx m_{\tilde{s}_{L}} \approx m_{\tilde{q}} \\
& m_{\tilde{\nu}_{e L}} \approx m_{\tilde{e}_{L}} \approx m_{\tilde{\nu}_{\mu L}} \approx m_{\tilde{\mu}_{L}} \approx m_{\tilde{\nu}_{\tau L}} \approx m_{\tilde{\tau}_{L}} \approx m_{\tilde{l}} .
\end{aligned}
$$

As will be discussed at length below, chargino production and decay are highly insensitive to the masses of all other scalars, and we may therefore set all squark masses to $m_{\tilde{q}}$ and all slepton masses to $m_{\tilde{l}}$ without loss of generality.

(g) The gaugino masses $M_{i}$ and the parameters $\mu$ and $\tan \beta$ may be taken to be real, so that $\mathrm{CP}$ violation plays no role in chargino events.

(h) The one-loop corrections to particle masses, chargino production, and chargino decay do not introduce any large new dependences on fundamental SUSY parameters.

(i) The parameters $M_{1}$ and $M_{2}$ are independent, i.e., we do not assume gaugino mass unification, as we are hoping to test this prediction of grand unified theories.

With the assumptions listed, our analysis is applicable to the bulk of parameter space available for study at LEP II. However, there are a number of small regions in the allowed parameter space where the physics is qualitatively different from the norm, and these will require special treatment. Since our principal aim is to explore the most general properties of chargino production, we will not study these exceptional regions, though their existence will be noted in the remarks below. In Sec. IIIG we will briefly discuss ways in which the unusual physics present in these cases might be detected.

Assumption (a) is commonly made in supersymmetry studies, as it prevents protons from decaying too quickly. Given R-parity conservation, the LSP is stable and must be among the decay products of any sparticle. The LSP must be uncolored and uncharged, and in many models it is the lightest neutralino $\tilde{\chi}_{1}^{0}$, as we have assumed in (b).

Because the LSP is very weakly interacting and unobservable in detectors, the first potentially observable SUSY signal must include the production of other light sparticles. As we would like to study the question of how much information can be obtained from the chargino signal alone, we will limit ourselves to models in which the sleptons and squarks have masses beyond the kinematic limit of LEP II, as given in (c). This assumption, along with the small cross sections for gluino production at $e^{+} e^{-}$colliders, implies that any reasonably large SUSY signal at LEP II must involve either the lighter chargino $\tilde{\chi}_{1}^{ \pm}$or the second lightest neutralino $\tilde{\chi}_{2}^{0}$. Although it would simplify our analysis, we cannot assume that the second lightest neutralino is heavier than the lighter chargino, since, as can be seen from Eqs. (4) and (ब), $m_{\tilde{\chi}_{2}^{0}}$ is not independent of $m_{\tilde{\chi}_{1}^{ \pm}}$and $m_{\tilde{\chi}_{1}^{0}}$. In fact, in the region of parameter space in which chargino production is accessible to LEP II, $\tilde{\chi}_{1}^{ \pm}$and $\tilde{\chi}_{2}^{0}$ are very roughly degenerate, 
with the mass difference typically in the range $-10 \mathrm{GeV} \lesssim m_{\tilde{\chi}_{2}^{0}}-m_{\tilde{\chi}_{1}^{ \pm}} \lesssim 20 \mathrm{GeV}$. When $m_{\tilde{\chi}_{2}^{0}}<m_{\tilde{\chi}_{1}^{ \pm}}$, it is possible for the lighter chargino to decay through a cascade decay, in which it decays to a $\tilde{\chi}_{2}^{0}$, which in turn decays to an LSP. If the mass splitting $m_{\tilde{\chi}_{1}^{ \pm}}-m_{\tilde{\chi}_{1}^{0}}$ is small, or direct decays to the LSP are suppressed by small couplings, the branching fraction for chargino cascade decays may be non-negligible. However, as the coincidence of these conditions occurs only in a small fraction of parameter space, we will not consider these decays further.

Under our assumptions, charginos decay to three-body final states consisting of an LSP and either two quarks or two leptons. The current lower bound on $m_{\tilde{\chi}_{1}^{0}}$ is roughly $20 \mathrm{GeV}$ [11.12], and so for charginos produced at LEP II with $\sqrt{s}=190 \mathrm{GeV}, m_{\tilde{\chi}_{1}^{ \pm}}-m_{\tilde{\chi}_{1}^{0}}<75 \mathrm{GeV}<$ $M_{W}$. Along with assumptions (a) - (d), this implies that the $W$ bosons, squarks and sleptons in chargino decays must be virtual particles. The tree-level relation $m_{H^{ \pm}}>M_{W}$ implies the same for charged Higgs bosons. If the tree-level $H^{ \pm}$mass were very low and its one-loop corrections were large and negative, the two-body decay $\tilde{\chi}_{1}^{ \pm} \rightarrow H^{ \pm} \tilde{\chi}_{1}^{0}$ could occur, but we will assume that this is not the case.

We may now display the production and decay diagrams of charginos. Chargino production from $e^{+} e^{-}$collisions is given by the three processes in Fig. 11 and includes $s$-channel $\gamma$ and $Z$ diagrams and $t$-channel $\tilde{\nu}_{e}$ exchange. Charginos decay to the LSP either hadronically through virtual $W$, squark, or $H^{ \pm}$diagrams,

$$
\tilde{\chi}^{+} \rightarrow\left(\tilde{\chi}_{1}^{0} W^{+*}, \tilde{d}^{*} u, \bar{d} \tilde{u}^{*}, \tilde{\chi}_{1}^{0} H^{+^{*}}\right) \rightarrow \tilde{\chi}_{1}^{0} \bar{d} u
$$

where $u(d)$ represents an up- (down-) type quark, or leptonically through virtual $W$, slepton, or $H^{ \pm}$diagrams,

$$
\tilde{\chi}^{+} \rightarrow\left(\tilde{\chi}_{1}^{0} W^{+^{*}}, \tilde{l}^{*} \nu, \bar{l} \tilde{\nu}^{*}, \tilde{\chi}_{1}^{0} H^{+*}\right) \rightarrow \tilde{\chi}_{1}^{0} \bar{l} \nu
$$

These decays are shown in Fig. 2. As each chiral fermion has its own complex scalar partner, charginos decay hadronically through six channels and leptonically through five (since there is no right-handed sneutrino).

Charginos are too short-lived to be directly observed, so we must infer everything from their decay products. It will be convenient to refer to the different types of chargino events by their decay modes. However, since a $\tau$ lepton produced in the leptonic decay of a chargino may itself decay either hadronically or leptonically, we must distinguish between the particles that are directly produced at the chargino decay vertices and those that are actually observed in the detector. To be precise, we establish the following terminology. If both charginos decay through the hadronic diagrams of Fig. 2, we will call the event a "hadronic mode" event. Events where both charginos decay through the leptonic diagrams of Fig. 2 will be called "leptonic mode" events, and those where one chargino decays through a leptonic diagram and one through a hadronic diagram will be called "mixed mode" events. On the other hand, if we wish to group chargino events by their observed final state, we will explicitly refer to the final state partons, using the notation $2 l, 2 j+l$, and $4 j$ for two lepton, dijet plus lepton, and four jet final states, respectively. In our notation, we will denote the final state of a hadronically decaying $\tau$ lepton as $j j$. For example, $2 j+l$ events will include leptonic mode events in which one chargino decays to a $\tau$ that decays hadronically and the other chargino decays to a $\bar{\tau}$ that decays leptonically. 
We will also need to identify the subset of $2 j+l$ events that are mixed mode events, that is, the $2 j+l$ events in which the hadrons do not come from a $\tau$. These events will be called "Y mode" events, the " $Y$ " representing the topology of the lepton track and the two jets. Hadronically decaying $\tau$ leptons produce a collimated hadronic system of low invariant mass and, often, just a single charged prong. In contrast, we will see that few dijet systems from chargino decays have a low invariant mass, and so it is usually possible to separate $Y$ events from the $2 j+l$ events resulting from hadronic $\tau$ decays. As will be discussed below, lepton universality (which follows from assumptions (e) and (f)) implies that a measurement of the number of $Y$ mode events can be directly converted to a measurement of the number of mixed mode events.

Before evaluating the importance of the various chargino decay diagrams, we digress slightly to consider the possibility of studying other SUSY signals. We have assumed that sleptons and squarks are beyond the kinematic limit of LEP II. If sleptons and squarks are within reach, they clearly will also give valuable information and will improve the results we obtain here. (Of course, if sleptons or squarks are not only within reach of LEP II, but are also less massive than the lighter chargino, the chargino will decay to two-body states containing these particles, and a modified analysis will be necessary.) It is more important to consider neutralino production. As noted above, $\tilde{\chi}_{1}^{ \pm}$and $\tilde{\chi}_{2}^{0}$ are very roughly degenerate, and therefore, if it is possible to produce $\tilde{\chi}_{1}^{+} \tilde{\chi}_{1}^{-}$chargino pairs, it is likely that $\tilde{\chi}_{2}^{0}$ pairs can be produced, and, in almost all cases, production of $\tilde{\chi}_{1}^{0} \tilde{\chi}_{2}^{0}$ is kinematically allowed. If $M_{2} \sim|\mu| \sim M_{W}, \tilde{\chi}_{3}^{0}$ or even $\tilde{\chi}_{4}^{0}$ production may be possible at LEP II. In principle neutralino pair production should also provide valuable information. However, neutralino production cross sections are typically significantly smaller than those for charginos and may differ by as much as an order of magnitude or more in some regions of parameter space [6, 70,20]. Furthermore, studies of $\tilde{\chi}_{1}^{0} \tilde{\chi}_{2}^{0}$ production at $\sqrt{s}=190 \mathrm{GeV}$ have concluded that the signal suffers from a large background from $W W$ production in both hadronic and leptonic modes [6.77. For these reasons we will not consider neutralino events further, other than to discuss their impact on our ability to isolate the chargino signal. Insofar as additional information about SUSY parameters can be obtained from slepton, squark, and neutralino signals at LEP II, the results of our study may be considered conservative.

From Figs. 1 and 2, it is clear that the chargino production and decay processes have a complex dependence on the various scalar masses, with the sneutrino mass $m_{\tilde{\nu}}$ entering the production process, and all slepton and squark masses entering the decay. In many versions of the MSSM, the slepton and squark masses are assumed to be unified at a high energy scale. When they are run down to low energies, typically the squarks acquire a greater mass than the sleptons through their QCD interactions. In principle, one would like to test this assumption. Unfortunately, without some simplifications, the large number of independent squark and slepton masses quickly complicates the analysis. We will make some simple assumptions to bring this dependence under control.

It is first important to note that with the assumption of negligible intergenerational mixings (assumption (e)), chargino events at LEP II are highly insensitive to certain scalar masses, namely, those of the third generation squarks, the right-handed squarks and sleptons, and the charged Higgs boson. To see this, we must discuss the scalar mass spectrum in greater detail. Sfermion masses are given by the following matrices: 


$$
\begin{gathered}
\mathbf{M}_{\tilde{u}}^{2}=\left(\begin{array}{cc}
m_{\tilde{Q}}^{2}+m_{u}^{2}+M_{Z}^{2}\left(\frac{1}{2}-\frac{2}{3} \sin ^{2} \theta_{W}\right) \cos 2 \beta & m_{u}\left(A_{u}-\mu \cot \beta\right) \\
m_{u}\left(A_{u}-\mu \cot \beta\right) & m_{\tilde{U}}^{2}+m_{u}^{2}+M_{Z}^{2}\left(\frac{2}{3} \sin ^{2} \theta_{W}\right) \cos 2 \beta
\end{array}\right), \\
\mathbf{M}_{\tilde{d}}^{2}=\left(\begin{array}{cc}
m_{\tilde{Q}}^{2}+m_{d}^{2}-M_{Z}^{2}\left(\frac{1}{2}-\frac{1}{3} \sin ^{2} \theta_{W}\right) \cos 2 \beta & m_{d}\left(A_{d}-\mu \tan \beta\right) \\
m_{d}\left(A_{d}-\mu \tan \beta\right) & m_{\tilde{D}}^{2}+m_{d}^{2}-M_{Z}^{2}\left(\frac{1}{3} \sin ^{2} \theta_{W}\right) \cos 2 \beta
\end{array}\right), \\
\mathbf{M}_{\tilde{e}}^{2}=\left(\begin{array}{cc}
m_{\tilde{L}}^{2}+m_{e}^{2}-M_{Z}^{2}\left(\frac{1}{2}-\sin ^{2} \theta_{W}\right) \cos 2 \beta & m_{e}\left(A_{e}-\mu \tan \beta\right) \\
m_{e}\left(A_{e}-\mu \tan \beta\right) & m_{\tilde{E}}^{2}+m_{e}^{2}-M_{Z}^{2}\left(\sin ^{2} \theta_{W}\right) \cos 2 \beta
\end{array}\right), \\
\mathbf{M}_{\tilde{\nu}}^{2}=m_{\tilde{L}}^{2}+\frac{1}{2} M_{Z}^{2} \cos 2 \beta,
\end{gathered}
$$

where the two-by-two matrices are in the basis $\left(\tilde{f}_{L}, \tilde{f}_{R}\right)$. The masses $m_{\tilde{Q}}, m_{\tilde{U}}, m_{\tilde{D}}, m_{\tilde{L}}$, and $m_{\tilde{E}}$ are the soft SUSY-breaking scalar masses of Eq. (2), and the $A_{i}$ are the coefficients of the SUSY-breaking cubic scalar terms. The mass matrices of the other two generations are identical in form. In the top and bottom squark sectors, the off-diagonal terms of the mass matrices can be large, leading to large left-right mass splittings and light top and bottom squarks. However, because the charginos we are studying are lighter than the top quark, and because we have assumed that intergenerational mixings are negligible, decays of charginos through third generation squarks are heavily suppressed. Thus, peculiarities of the third generation are irrelevant for our analysis. For all other squarks and sleptons, left-right mixings are usually negligible and the masses of the sparticles are given by the diagonal elements of the matrices. An exception occurs when $m_{\tau}\left(A_{\tau}-\mu \tan \beta\right) \sim m_{\tilde{L}}^{2}$, and similarly for muons and strange quarks, which is possible in certain corners of SUSY parameter space (see Sec. IID). However, we will ignore left-right mixing in this study and merely note in Sec. IIIG that it would have observable consequences.

Let us now consider the right-handed scalars of the three slepton and first two squark generations. Right-handed scalars couple only to the Higgsino component of the chargino. As these couplings are the supersymmetric analogues of Higgs couplings, they are suppressed by either $m_{u} /\left(M_{W} \sin \beta\right)$ or $m_{d} /\left(M_{W} \cos \beta\right)$, where $m_{u}$ and $m_{d}$ are the masses of the corresponding standard model fermion. Such couplings are important only for extreme values of $\tan \beta$, and so for almost all of parameter space, chargino events are insensitive to the masses of right-handed scalars. The $H^{ \pm}$amplitude is suppressed by similar couplings, and may also be ignored.

Thus, only the scalar masses listed in Eq. (7) are relevant. Left-handed scalars couple to charginos through their gaugino components, and these couplings are not suppressed. However, in any given generation, the left-handed squarks (sleptons) belong to the same SU(2) doublet, and so have the same soft SUSY-breaking mass term $m_{\tilde{Q}}\left(m_{\tilde{L}}\right)$. Their masses are therefore split only by the last terms of the diagonal entries of the scalar mass matrices, the $D$ terms, which induce a mass splitting that is typically of order $20 \mathrm{GeV}$ or less for the masses we will consider. Such splittings are not important for this study, and so, within each generation, the left-handed squarks and sleptons may be taken to be roughly degenerate. Furthermore, there are bounds on slepton and squark non-degeneracy between different generations from $\mu \rightarrow e \gamma$ and flavor changing neutral current constraints 
[33]. Motivated by these considerations, we adopt assumption (f). Because the scalar masses that are not constrained in assumption (f) are irrelevant for chargino events at LEP II, we may set them to any reasonable values. For convenience only, we will assume throughout our study that all sleptons have the same mass $m_{\tilde{l}}=m_{\tilde{\nu}}$, and similarly that all squarks have mass $m_{\tilde{q}}$; it should be remembered, however, that our results depend only on the weaker assumption (f).

Throughout this study, we will assume that one-loop corrections do not greatly affect our analysis (assumption (h)). Studies have shown that one-loop effects on chargino and neutralino masses are generically only a few percent [34, 35], and so we do not expect this assumption to be very restrictive. Even if one-loop corrections are substantial, as long as they do not introduce qualitatively new dependences on SUSY parameters into the observables we use, the analysis presented in this study will still be applicable without large modifications.

Often gauge coupling constants and gaugino masses are assumed to unify at some high scale. This assumption implies that even at lower energy scales we have (to the accuracy of one-loop renormalization group equations) [36]

$$
\frac{M_{2}}{g_{2}^{2}}=\frac{3}{5} \frac{M_{1}}{g_{1}^{2}}=\frac{M_{3}}{g_{3}^{2}}
$$

or approximately $M_{1} \approx \frac{1}{2} M_{2}$ and $M_{3} \approx \frac{10}{3} M_{2}$ at $M_{Z}$. We may ignore the gluino mass $M_{3}$, since, given assumption (d), gluinos enter chargino production and decay only through loop diagrams, which are likely to be small. As noted previously, since one of our main goals is to test the unification of $M_{1}$ and $M_{2}$, we will avoid assuming a universal gaugino mass and will take these parameters to be independent (assumption (i)). It is possible without loss of generality to set $M_{2} \geq 0$, and we will follow this convention. Without the gaugino mass unification assumption, however, $M_{1}$ may be either positive or negative.

\section{Regions of Parameter Space}

Given the discussion above, the SUSY parameter space of the MSSM that is relevant to our study of chargino production is given by the six parameters $\left(\mu, M_{2}, \tan \beta, M_{1}, m_{\tilde{l}}, m_{\tilde{q}}\right)$. We can now examine the regions of parameter space for which chargino production is kinematically allowed at LEP II with $\sqrt{s}=190 \mathrm{GeV}$. The chargino mass $m_{\tilde{\chi}_{1}^{ \pm}}$is completely determined by the three parameters $\mu, M_{2}$, and $\tan \beta$. In Fig. 3, contours of constant $m_{\tilde{\chi}_{1}^{ \pm}}$ in the $\left(\mu, M_{2}\right)$ plane are plotted for fixed $\tan \beta=4$; the contours are similar for other values of $\tan \beta$. The cross-hatched regions along the $M_{2}=0$ and $\mu=0$ axes are experimentally excluded by lower limits on sparticle masses [11,12], and the hatched region is the inaccessible region where $m_{\tilde{\chi}_{1}^{ \pm}}>95 \mathrm{GeV}$. In Fig. 国 we plot constant $m_{\tilde{\chi}_{1}^{0}}$ contours for $\tan \beta=4$ and, since $m_{\tilde{\chi}_{1}^{0}}$ depends on $M_{1}$, three different values of $M_{1} / M_{2}$. (For these plots, only the experimental limits $m_{\tilde{\chi}_{1}^{0}}>20 \mathrm{GeV}$ and $m_{\tilde{\chi}_{1}^{ \pm}}>45 \mathrm{GeV}$ have been included, as the other chargino and neutralino mass limits assume gaugino mass unification [11, 12]. Inclusion of the mass bounds for the other neutralinos and chargino in the case $M_{1}=\frac{1}{2} M_{2}$ extends the excluded region only slightly. The exact shape of the experimentally excluded region will be unimportant for this study.) 
It is convenient to further divide the $\left(\mu, M_{2}\right)$ plane into regions based on the gaugino contents of the light gauginos. To quantify this, we define the gaugino contents of the lighter chargino and LSP to be 30.

$$
\begin{aligned}
\rho_{\tilde{\chi}_{1}^{ \pm}} & \equiv\left|\mathbf{V}_{11}\right|^{2} \\
\rho_{\tilde{\chi}_{1}^{0}} & \equiv\left|\mathbf{N}_{11}\right|^{2}+\left|\mathbf{N}_{12}\right|^{2} .
\end{aligned}
$$

(We have arbitrarily chosen to define $\rho_{\tilde{\chi}_{1}^{ \pm}}$in terms of $\mathbf{V}_{11}$ instead of $\mathbf{U}_{11}$. These differ little throughout parameter space, and for the purposes of defining $\rho_{\tilde{\chi}_{1}^{ \pm}}$, the discrepancy is not important.) The variables $\rho_{\tilde{\chi}_{1}^{ \pm}}$and $\rho_{\tilde{\chi}_{1}^{0}}$ lie in the range $0 \leq \rho_{\tilde{\chi}_{1}^{ \pm}}, \rho_{\tilde{\chi}_{1}^{0}} \leq 1 ; \rho_{\tilde{\chi}_{1}^{ \pm}}\left(\rho_{\tilde{\chi}_{1}^{0}}\right)$ is zero when $\tilde{\chi}_{1}^{+}\left(\tilde{\chi}_{1}^{0}\right)$ is pure Higgsino and is one when $\tilde{\chi}_{1}^{+}\left(\tilde{\chi}_{1}^{0}\right)$ is pure gaugino. Although they may differ substantially in certain regions of parameter space, $\rho_{\tilde{\chi}_{1}^{ \pm}}$and $\rho_{\tilde{\chi}_{1}^{0}}$ are correlated: when $M_{2},\left|M_{1}\right| \ll|\mu|$, both $\tilde{\chi}_{1}^{ \pm}$and $\tilde{\chi}_{1}^{0}$ are essentially gaugino states, while in the opposite limit, $M_{2},\left|M_{1}\right| \gg|\mu|$, they are both dominated by their Higgsino components. We will present results for both quantities, and will find that the bounds we obtain for them are roughly the same. The quantity $\rho_{\tilde{\chi}_{1}^{0}}$ has implications for the viability of the LSP as a dark matter candidate. As shown in a number of studies [30], $\tilde{\chi}_{1}^{0}$ is a good dark matter candidate when it is gaugino-like with $\rho_{\tilde{\chi}_{1}^{0}} \gtrsim 0.9$. For $\rho_{\tilde{\chi}_{1}^{0}} \lesssim 0.9$, the LSPs annihilate so quickly in the early universe that they provide insufficient mass today to be interesting dark matter candidates. The gaugino content $\rho_{\tilde{\chi}_{1}^{0}}$ is therefore an important parameter for us to determine. However, because $\rho_{\tilde{\chi}_{1}^{ \pm}}$depends only on the three parameters $\mu, M_{2}$, and $\tan \beta$, it is the more convenient of the two quantities to use to divide the parameter space. Contours of equal $\rho_{\tilde{\chi}_{1}^{ \pm}}$are plotted in Fig. 5. Although the specific boundaries are not particularly important, for definiteness we will refer to the region with $\rho_{\tilde{\chi}_{1}^{ \pm}} \geq 0.9$ as the gaugino region, the region with $\rho_{\tilde{\chi}_{1}^{ \pm}} \leq 0.2$ as the Higgsino region, and the region with $0.2<\rho_{\tilde{\chi}_{1}^{ \pm}}<0.9$ as the mixed region. With these definitions, roughly speaking, $\tilde{\chi}_{1}^{0}$ is a good dark matter candidate in the gaugino region, but is not a viable candidate in the mixed and Higgsino regions.

It is evident from Figs. 3, 4, and 5 that accurate determinations of $m_{\tilde{\chi}_{1}^{ \pm}}$and $m_{\tilde{\chi}_{1}^{0}}$ are not enough to determine the gaugino content $\rho_{\tilde{\chi}_{1}^{ \pm}}$. While a measurement of $m_{\tilde{\chi}_{1}^{0}} \approx \frac{1}{2} m_{\tilde{\chi}_{1}^{ \pm}}$ might be taken as evidence that the SUSY parameters lie in the gaugino region and that gaugino masses unify, this is not the only possibility. For example, the masses $m_{\tilde{\chi}_{1}^{ \pm}} \approx$ $80 \mathrm{GeV}$ and $m_{\tilde{\chi}_{1}^{0}} \approx 40 \mathrm{GeV}$ can be obtained with the parameters $\left(\mu, M_{2}, \tan \beta, M_{1} / M_{2}\right)=$ $(-400,75,4,0.5)$ in the gaugino region with $\rho_{\tilde{\chi}_{1}^{ \pm}}=1.00$, and also with the parameters $(-78,170,4,0.25)$ in the mixed region with $\rho_{\tilde{\chi}_{1}^{ \pm}}=0.34$. As will be seen below, more careful analysis can differentiate between such possibilities.

We have now found the regions in which chargino production is allowed. However, if the splitting between the masses of the chargino and the LSP is very small, and the charginos are produced with low velocity, the chargino decay products will have very low energy in the laboratory frame and may be too soft to be experimentally useful. The approximate relations 37

$$
m_{\tilde{\chi}_{1}^{0}} \approx \min \left\{|\mu|, M_{2},\left|M_{1}\right|\right\} \quad \text { and } \quad m_{\tilde{\chi}_{1}^{ \pm}} \approx \min \left\{|\mu|, M_{2}\right\}
$$

are valid in the far Higgsino and far gaugino regions. With these in mind, it is easy to see that for increasing $M_{2}$ and fixed $M_{1} / M_{2}$ in the Higgsino region, $\tilde{\chi}_{1}^{ \pm}$and $\tilde{\chi}_{1}^{0}$ become more 
and more degenerate. The maximum and minimum energies for dijet or $l \nu$ systems from chargino events are

$$
E^{\max , \min }=\frac{E_{b}}{2}\left[1 \pm\left(1-\frac{m_{\tilde{\chi}_{1}^{ \pm}}^{2}}{E_{b}^{2}}\right)^{\frac{1}{2}}\right]\left[1-\frac{m_{\tilde{\chi}_{1}^{0}}^{2}}{m_{\tilde{\chi}_{1}^{ \pm}}^{2}}\right]
$$

where $E_{b}$ is the beam energy. The maximum energy for single jets and leptons is also given by $E^{\max }$, which is plotted in Fig. 6 for the case $M_{1} / M_{2}=0.5, \tan \beta=4$. We see that, in this case, the far Higgsino region with $M_{2} \gtrsim 500 \mathrm{GeV}$, contains areas in which all decay products have energies $\lesssim 10 \mathrm{GeV}$. Thus, in these regions chargino production may be visible but difficult to use in precision studies. As $M_{2}$ increases further, it becomes difficult even to detect the chargino signal above background. The problem of soft decay products is generic only in the far Higgsino region. In all other regions within the bands shown in Fig. 3, as long as $m_{\tilde{\chi}_{1}^{ \pm}}$lies somewhat below the beam energy, and the splitting between $m_{\tilde{\chi}_{1}^{ \pm}}$and $m_{\tilde{\chi}_{1}^{0}}$ is not anomalously small, chargino production can be observed and studied at LEP II.

For extremely large values of $M_{2}$, the lifetime of the chargino becomes long, and one might hope to tag charginos by looking for tracks which do not intersect the interaction point. From the formula for the chargino decay width presented later in Eq. (49), one may estimate the chargino decay length, which is of order the impact parameter for these tracks. Roughly, for charginos to travel 10 (100) $\mu \mathrm{m}$ before decaying requires $M_{2} \gtrsim 2(3) \mathrm{TeV}$. Such large values of $M_{2}$ are disfavored by fine-tuning considerations, as discussed below.

\section{Boundaries of Parameter Space}

In this subsection, we specify the boundary of the region of parameter space that we will investigate. The six SUSY parameters may be restricted on the basis of fine-tuning prejudices and other considerations. We discuss each parameter in turn.

As noted in Sec. I, if SUSY is to naturally explain the electroweak scale, the SUSYbreaking masses $M_{1}$ and $M_{2}$ must be less than or of order $1 \mathrm{TeV}$. In fact, if charginos are discovered, the parameter $M_{1}$ may be further bounded. Eq. (16) implies that in the far gaugino and far Higgsino regions, if $M_{1} \gtrsim M_{2}, \tilde{\chi}_{1}^{ \pm}$and $\tilde{\chi}_{1}^{0}$ are virtually degenerate. Thus, the condition that $m_{\tilde{\chi}_{1}^{ \pm}}-m_{\tilde{\chi}_{1}^{0}}$ be large enough that the decay products are detectable implies to a good approximation that $\left|M_{1}\right|<M_{2}$, and so we will also impose this constraint. Although $\mu$ is not a SUSY-breaking parameter, fine-tuning considerations constrain it to lie at or below the $\mathrm{TeV}$ scale.

The parameters $m_{\tilde{l}}$ and $m_{\tilde{q}}$ are also SUSY-breaking masses, and therefore must also be less than or of order $1 \mathrm{TeV}$. Sleptons and squarks with masses of $1 \mathrm{TeV}$ are effectively decoupled and are indistinguishable from those with infinite mass. We therefore limit the analysis to $m_{\tilde{l}}, m_{\tilde{q}} \leq 1 \mathrm{TeV}$. As we are considering the scenario in which only charginos are produced at LEP II, we take $m_{\tilde{l}} \geq 100 \mathrm{GeV}$. The squark mass lower bound from hadron colliders is likely to be approximately $150 \mathrm{GeV}$ when LEP II begins operation, and we therefore take this as the lower bound on $m_{\tilde{q}}$.

The quantity $\tan \beta$ is more difficult to delimit. If one assumes the desert hypothesis, applies the MSSM renormalization group equations to the Higgs-fermion Yukawa couplings, 
and demands that the couplings remain finite up to a scale $\Lambda=10^{16} \mathrm{GeV}$, one finds that for the present top quark mass measurement of $m_{t}=174 \pm 10_{-12}^{+13} \mathrm{GeV}$ [38], the bounds on $\tan \beta$ are $1 \lesssim \tan \beta \lesssim 50$. We will adopt these limits.

In summary, given the assumptions above, our task is to explore and restrict the sixdimensional SUSY parameter space given by

$$
\begin{aligned}
-1 \mathrm{TeV} & \lesssim \mu \\
0 & \leq M_{2} \lesssim 1 \mathrm{TeV} \\
1 & \leq \tan \beta \leq 50 \\
-M_{2} & \leq M_{1} \leq M_{2} \\
100 \mathrm{GeV} & \leq m_{\tilde{l}} \leq 1 \mathrm{TeV} \\
150 \mathrm{GeV} & \leq m_{\tilde{q}} \leq 1 \mathrm{TeV} .
\end{aligned}
$$

\section{OBSERVABLES OF CHARGINO PRODUCTION}

In this section, we will discuss the observables that we will use to restrict SUSY parameter space. As stated earlier, our goal is to gain an understanding of chargino pair production by using as much analytic information as possible. For this reason we will not study observables for which no analytic formulae can easily be found, such as the distribution of lepton energies in $2 j+l$ events, even though these quantities contain information which is not accessible through the observables we consider. It is therefore probable that a global likelihood fit to the data will be able to put tighter bounds on supersymmetry parameter space than we will claim below. In this sense, our results are conservative.

The four quantities that will be central to our analysis are the chargino and neutralino masses, the total cross section for chargino production, and the leptonic branching fraction:

$$
\begin{aligned}
& m_{\tilde{\chi}_{1}^{ \pm}}\left(\mu, M_{2}, \tan \beta\right), \\
& m_{\tilde{\chi}_{1}^{0}}\left(\mu, M_{2}, \tan \beta, M_{1}\right), \\
& \sigma_{\text {total }}\left(\mu, M_{2}, \tan \beta, m_{\tilde{\nu}}=m_{\tilde{l}}\right), \\
& B_{l}\left(\mu, M_{2}, \tan \beta, M_{1}, m_{\tilde{l}}, m_{\tilde{q}}\right) \equiv \frac{\Gamma\left(\tilde{\chi}^{+} \rightarrow \tilde{\chi}^{0} \bar{l} \nu\right)}{\Gamma\left(\tilde{\chi}^{+} \rightarrow \text { anything }\right)} .
\end{aligned}
$$

Of course, four observables will not allow us to determine six parameters, but, as will be seen in Sec. $\mathrm{\nabla}$, these four observables can often restrict the parameter space to a region in which the quantities of greatest interest are already somewhat constrained. The forwardbackward asymmetry of chargino production $A_{F B}^{\tilde{\chi}^{ \pm}}$will also be discussed, but for a number of reasons to be mentioned below, we will not use this quantity directly. No other variables were found that could be studied without performing Monte Carlo simulations at a large number of points in parameter space. The left-right asymmetry in the production cross section requires polarized electron beams and is inaccessible at LEP II, but has implications for chargino production at threshold and will also be discussed below.

In the following subsections, we will consider each observable, first analyzing its dependence on the underlying SUSY parameters, and then discussing the method by which it may be extracted from chargino event samples. This section will be confined to theoretical considerations; experimental issues will be discussed in Sec. IV. In particular, discussion of 
issues involving experimental efficiencies and minor subtleties involving the hadronic decays of the $\tau$ lepton will be deferred to the following sections. In Sec. $\mathrm{V}$, the measurements suggested in this section will be applied to Monte Carlo simulation case studies at specific points in parameter space, and results will be obtained with cuts, finite detector resolution, and finite statistics included.

\section{A. Chargino and Neutralino Masses}

The chargino mass $m_{\tilde{\chi}_{1}^{ \pm}}\left(\mu, M_{2}, \tan \beta\right)$ and the LSP mass $m_{\tilde{\chi}_{1}^{0}}\left(\mu, M_{2}, \tan \beta, M_{1}\right)$ are sensitive to fundamental parameters of supersymmetry and are relatively easy to determine. Their dependences on the underlying SUSY parameters were discussed in Sec. II. Here we note only that in the gaugino region $m_{\tilde{\chi}_{1}^{ \pm}} \approx M_{2}$ and $m_{\tilde{\chi}_{1}^{0}} \approx\left|M_{1}\right|$, and the masses are therefore directly sensitive to two fundamental parameters. In contrast, both masses are close to $|\mu|$ in the Higgsino region (unless $\left|M_{1}\right|<|\mu|$, in which case the LSP can have a mass near $\left.\left|M_{1}\right|\right)$ and the mass splitting $m_{\tilde{\chi}_{1}^{ \pm}}-m_{\tilde{\chi}_{1}^{0}}$ is a complicated function of several parameters.

The masses $m_{\tilde{\chi}_{1}^{ \pm}}$and $m_{\tilde{\chi}_{1}^{0}}$ can be measured in chargino events in at least two ways. It is impossible to kinematically reconstruct chargino pair production events, since the charginos' decay products include two unobservable LSPs. However, because the unobserved LSPs are typically quite massive and carry off large energies and momenta, chargino events with two jets and an isolated lepton are easily separated from standard model backgrounds by a series of cuts, as we will see in Sec. IV. After imposing such cuts, one can find the dijet energy $E_{j j}$ and dijet mass $m_{j j}$ for each of the remaining events. The endpoints of the dijet energy and mass spectra are completely determined by $m_{\tilde{\chi}_{1}^{ \pm}}$and $m_{\tilde{\chi}_{1}^{0}}$, with the endpoints of the $E_{j j}$ spectrum given by Eq. (17), and the $m_{j j}$ distribution lying between zero and $m_{\tilde{\chi}_{1}^{ \pm}}-m_{\tilde{\chi}_{1}^{0}}$. If at least two of the three endpoints are sufficiently sharp to be well-measured, they can be used to precisely determine $m_{\tilde{\chi}_{1}^{ \pm}}$and $m_{\tilde{\chi}_{1}^{0}}$. Of course, detector and beam effects will smear the endpoints, but in Sec. $\mathrm{V}$ we will see that the masses may still be measured to a few GeV with this method.

An energy scan at the chargino production threshold provides an alternate determination of $m_{\tilde{\chi}_{1}^{ \pm}}$[29]. In Fig. 7, the total cross section as a function of $\sqrt{s}$ (solid curve) is plotted for the particular point in parameter space $\left(\mu, M_{2}, \tan \beta, m_{\tilde{\nu}}\right)=(-400,75,4,200)$. For comparison, a unit of $\mathrm{R}$ is also given (dashed curve). The sudden rise in cross section, characteristic of fermion production, makes possible a highly accurate determination of $m_{\tilde{\chi}_{1}^{ \pm}}$. Such behavior is common for all points in parameter space. Near threshold the charginos are nearly at rest, so the only unknown momentum in the decay $\tilde{\chi}_{1}^{ \pm} \rightarrow q \bar{q} \tilde{\chi}_{1}^{0}$ is that of the LSP. By reconstructing the hadronically decaying chargino in a mixed mode event, one obtains the mass of the LSP. Although this method is likely to provide a significantly more accurate determination of the chargino mass [29], we will not assume in our case studies that an energy scan will be performed, instead relying solely on the endpoint determinations.

\section{B. Differential Cross Section and Related Observables}

Next we study the differential cross section $\frac{d \sigma}{d \cos \theta}\left(\mu, M_{2}, \tan \beta, m_{\tilde{\nu}}\right)$ of chargino production and consider associated observables. As we will emphasize below, only two combinations of 
the parameters $\left(\mu, M_{2}, \tan \beta, m_{\tilde{\nu}}\right)$ can be easily extracted even theoretically. One of these is proportional to the total cross section $\sigma_{\text {total }}$, while the other is proportional to the production forward-backward asymmetry $A_{F B}^{\tilde{\chi}^{ \pm}}$. To a first approximation, which we will show to be sufficiently accurate, every other quantity that depends only on $\frac{d \sigma}{d \cos \theta}$ gives information which is equivalent to that contained in $\sigma_{\text {total }}$ and $A_{F B}^{\tilde{\chi}^{ \pm}}$. Unfortunately, with the exception of $\sigma_{\text {total }}$, none of these quantities is observable, since the direction of the charginos cannot be fully reconstructed. The angular distributions of the visible particles depend on the chargino decay vertices as well as on $\frac{d \sigma}{d \cos \theta}$, and although they are of great interest, they are not amenable to analytic study. They can be investigated by Monte Carlo simulations, but this is beyond the scope of the present work. Our approach therefore only allows us to extract a single combination of the four underlying parameters that determine the differential cross section.

We first present the differential cross section for chargino production and analyze its dependence on the various SUSY parameters in the region of parameter space given by Eq. (18). Formulae for the differential cross section and total cross section have been given in many previous studies [2, 3, 4, 5, 9, 28]. Here we present the differential cross section for completeness and in a form that allows us to highlight certain properties that have particular relevance to our analysis.

The cross section is built from the three ingredients in Fig. 1. The couplings in the virtual photon diagram are, of course, independent of SUSY parameters. To compute the virtual $Z$ diagram, we need the couplings of chargino currents to the $Z$. The virtual $Z$ amplitude for producing chargino states $\tilde{\chi}_{i}^{+}$and $\tilde{\chi}_{j}^{-}$is given by

$$
\begin{gathered}
\bar{v}\left(e^{+}\right) i g \gamma^{\mu} \frac{1}{\cos \theta_{W}}\left[\left(\frac{1}{2}-\sin ^{2} \theta_{W}\right) P_{L}+\left(-\sin ^{2} \theta_{W}\right) P_{R}\right] u\left(e^{-}\right) \times \\
\bar{u}\left(\tilde{\chi}_{i}^{+}\right) i g \gamma_{\mu} \frac{1}{\cos \theta_{W}}\left[\mathbf{O}_{\mathbf{L} i j}^{\prime} P_{L}+\mathbf{O}_{\mathbf{R} i j}^{\prime} P_{R}\right] u\left(\tilde{\chi}_{j}^{-}\right) \times \frac{-i}{s-M_{Z}^{2}},
\end{gathered}
$$

where the dependence on the SUSY parameters is through the quantities that are conventionally labeled $\mathbf{O}_{\mathbf{L} i j}^{\prime}$ and $\mathbf{O}_{\mathbf{R} i j}^{\prime}$ (see first reference in [1]). These are the combinations of $\mathbf{U}$ and $\mathbf{V}$ matrices given by

$$
\begin{aligned}
\mathbf{O}_{\mathbf{L} i j}^{\prime} & \equiv-\mathbf{V}_{i 1} \mathbf{V}_{j 1}^{*}-\frac{1}{2} \mathbf{V}_{i 2} \mathbf{V}_{j 2}^{*}+\delta_{i j} \sin ^{2} \theta_{W} \\
\mathbf{O}_{\mathbf{R} i j}^{\prime} & \equiv-\mathbf{U}_{i 1}^{*} \mathbf{U}_{j 1}-\frac{1}{2} \mathbf{U}_{i 2}^{*} \mathbf{U}_{j 2}+\delta_{i j} \sin ^{2} \theta_{W},
\end{aligned}
$$

where the indices $i, j$ are 1 for the lighter chargino and 2 for the heavier. As we will only be concerned with the lighter chargino, we define $O_{L}^{\prime} \equiv \mathbf{O}_{\mathbf{L} 11}^{\prime}$ and $O_{R}^{\prime} \equiv \mathbf{O}_{\mathbf{R} 11}^{\prime}$. After a Fierz transformation, the $\tilde{\nu}$ exchange diagram is also of a similar form, and its amplitude is given by

$$
-\frac{1}{2}\left|\mathbf{V}_{11}\right|^{2} \bar{v}\left(e^{+}\right) i g \gamma^{\mu} P_{L} u\left(e^{-}\right) \times \bar{u}\left(\tilde{\chi}_{i}^{+}\right) i g \gamma_{\mu} P_{L} u\left(\tilde{\chi}_{j}^{-}\right) \times \frac{-i}{t-m_{\tilde{\nu}}^{2}} .
$$

Combining these three contributions, one finds that the differential cross section for chargino production from unpolarized $e^{+} e^{-}$beams in units of $\mathrm{R}$ is given by

$$
\frac{d \sigma}{d x}=\sum_{i=L}^{R} \frac{3 v}{32}\left[R_{i}^{2}(1-v x)^{2}+S_{i}^{2}(1+v x)^{2}+2 R_{i} S_{i}\left(1-v^{2}\right)\right],
$$


where the sum is over the two $e^{-}$helicities, $v$ is the chargino velocity, and $x=\cos \theta$, the cosine of the angle between the positive chargino $\tilde{\chi}_{1}^{+}$and the positron beam. The variables $R_{L, R}$ and $S_{L, R}$ are

$$
\begin{aligned}
R_{L} & \equiv 1-K_{L} O_{L}^{\prime}-c_{\tilde{\nu}}, \\
S_{L} & \equiv 1-K_{L} O_{R}^{\prime}, \\
R_{R} & \equiv 1-K_{R} O_{R}^{\prime}, \\
S_{R} & \equiv 1-K_{R} O_{L}^{\prime},
\end{aligned}
$$

where $K_{L, R}$ are constants associated with $Z$ production from $e_{L, R}^{-}$,

$$
\begin{aligned}
& K_{L} \equiv \frac{s}{s-M_{Z}^{2}} \frac{1}{\sin ^{2} \theta_{W} \cos ^{2} \theta_{W}}\left(\frac{1}{2}-\sin ^{2} \theta_{W}\right), \\
& K_{R} \equiv \frac{s}{s-M_{Z}^{2}} \frac{1}{\sin ^{2} \theta_{W} \cos ^{2} \theta_{W}}\left(-\sin ^{2} \theta_{W}\right),
\end{aligned}
$$

and the $\tilde{\nu}$ diagram contribution is given by

$$
c_{\tilde{\nu}} \equiv \frac{2\left|\mathbf{V}_{11}\right|^{2}}{\sin ^{2} \theta_{W}\left[1-2 v x+v^{2}+4 m_{\tilde{\nu}}^{2} / s\right]} .
$$

We now analyze these formulae in some detail to determine what can be learned experimentally. We will show that (a) in most of the allowed parameter space only two combinations of $O_{L}^{\prime}, O_{R}^{\prime},\left|\mathbf{V}_{11}\right|^{2}$ and $m_{\tilde{\nu}}$ contribute significantly to the unpolarized cross section; (b) the quantities most sensitive to these two combinations are the total cross section and the forward-backward production asymmetry; and (c) no other quantities involving the unpolarized cross section can be found that add significantly to our knowledge. We will illustrate these points using a perturbative expansion of the differential cross section in variables which we will define below. While the expansion is not always valid, we have found numerically that the conclusions that we draw in the perturbative regime hold throughout the allowed parameter space.

We now identify the small quantities in which to do perturbation theory. We define

$$
\begin{gathered}
\bar{K} \equiv \frac{1}{2}\left(K_{L}+\left|K_{R}\right|\right) ; \kappa \equiv \frac{1}{2}\left(K_{L}-\left|K_{R}\right|\right) ; \\
\bar{O} \equiv \frac{1}{2}\left(O_{L}^{\prime}+O_{R}^{\prime}\right) ; \omega \equiv \frac{1}{2}\left(O_{L}^{\prime}-O_{R}^{\prime}\right) ; \\
\xi_{0} \equiv \frac{s}{m_{\tilde{\nu}}^{2}}
\end{gathered}
$$

From Eq. (25) we see that $K_{L} \approx-K_{R}$, so $\bar{K} \gg \kappa$. Specifically, for $\sqrt{s}=190 \mathrm{GeV}$, $\bar{K} \approx 1.8 \gg \kappa \approx 0.13$, independently of all SUSY parameters. From Sec. II and Eq. (21) it follows that $O_{L}^{\prime} \approx O_{R}^{\prime}$ in both the gaugino and Higgsino regions, so that in most cases $\bar{O}$, which runs between $-1+\sin ^{2} \theta_{W}$ in the gaugino region and $-1 / 2+\sin ^{2} \theta_{W}$ in the Higgsino region, has absolute value much larger than $\omega$, which is zero in the gaugino and Higgsino regions and whose absolute value never exceeds 0.2 . The last small quantity, $\xi_{0} \equiv s / m_{\tilde{\nu}}^{2}$, is small for sneutrino masses much larger than $200 \mathrm{GeV}$. Because of the form of Eq. (26), the expansion can be slightly improved by using not $\xi_{0}$ but 


$$
\xi \equiv \frac{\xi_{0}}{1+\left(1+v^{2}\right) \xi_{0} / 4} .
$$

In the perturbative regime, the unpolarized differential cross section $d \sigma / d x$ is conveniently written

$$
\frac{d \sigma}{d x}=\frac{3 v}{32} \sum_{k=0}^{\infty} x^{k} A_{k},
$$

where, to first order in $\xi, \kappa$ and $\omega$,

$$
\begin{aligned}
& A_{0} \approx 4\left(2-v^{2}\right)\left[1+\bar{K}^{2} \bar{O}^{2}-2 \bar{O} \kappa-\frac{\left|\mathbf{V}_{11}\right|^{2}}{4 \sin ^{2} \theta_{W}}(1-\bar{K} \bar{O}) \xi\right], \\
& A_{1} \approx 16 v\left[\bar{K} \omega+\frac{\left|\mathbf{V}_{11}\right|^{2}}{8 \sin ^{2} \theta_{W}}(1-\bar{K} \bar{O}) \xi\right], \\
& A_{2} \approx \frac{v^{2}}{2-v^{2}} A_{0} \\
& A_{k} \sim \mathcal{O}\left(\frac{\left|\mathbf{V}_{11}\right|^{2}}{4 \sin ^{2} \theta_{W}} v^{k} \xi^{k-1}\right), k>2 .
\end{aligned}
$$

(Recall that $\left|\mathbf{V}_{11}\right|^{2} \leq 1$.) Thus, to a first approximation it is impossible to measure separately the four quantities of interest $-\bar{O}, \omega,\left|\mathbf{V}_{11}\right|^{2}$, and $\xi$ - since the cross section effectively depends only on $A_{0} \propto A_{2}$ and $A_{1}$. As can easily be seen from the angular dependence of Eq. (29), these coefficients are most sensitively probed by the total cross section

$$
\sigma_{\text {total }} \approx \frac{v}{8}\left(\frac{3-v^{2}}{2-v^{2}}\right) A_{0}
$$

and the forward-backward asymmetry

$$
A_{F B}^{\tilde{\chi}^{ \pm}} \approx \frac{3 A_{1}}{4 A_{0}}\left(\frac{2-v^{2}}{3-v^{2}}\right) .
$$

The quantities $A_{k}$ for $k>2$ are greatly suppressed in the perturbative regime and cannot give additional useful information.

Perhaps surprisingly, the basic conclusions of this perturbative analysis are correct for the entire accessible region of parameter space. The coefficients $A_{k}, k>2$, become substantial and the relation $A_{0} \propto A_{2}$ breaks down only for $\left|\mathbf{V}_{11}\right|$ near one and for large $\xi$, that is, in the gaugino region with a sneutrino mass near $100 \mathrm{GeV}$. However, even in this case we find numerically that it is extremely difficult to extract additional information from the differential cross section. For example, consider a simple variable which is orthogonal to $A_{F B}^{\tilde{\chi}^{ \pm}}$and linearly independent of $\sigma_{\text {total }}$ :

$$
A_{\text {central }}^{\tilde{\chi}^{ \pm}}=\frac{\int_{-1 / 2}^{1 / 2} d x \frac{d \sigma}{d x}}{\sigma_{\text {total }}} .
$$


In the perturbative regime this variable is simply

$$
A_{\text {central }}^{\tilde{\chi}^{ \pm}} \approx A_{\text {central }}^{(0) \tilde{\chi}^{ \pm}} \equiv \frac{1}{16} \frac{24-11 v^{2}}{3-v^{2}}
$$

which follows from Eqs. (29) and (30). Deviations from this prediction would provide new information beyond $\sigma_{\text {total }}$ and $A_{F B}^{\tilde{\chi}^{ \pm}}$. In Fig. 8 the ratio $A_{\text {central }}^{\tilde{\chi}^{ \pm}} / A_{\text {central }}^{(0) \tilde{\chi}^{ \pm}}$is shown for two light sneutrino masses $m_{\tilde{\nu}}=200 \mathrm{GeV}$ and $100 \mathrm{GeV}$. The figure indicates that $A_{\text {central }}^{\tilde{\chi}^{ \pm}}$is within a few percent of the perturbative prediction everywhere except in the gaugino region for extremely small sneutrino mass and large chargino mass, where a ten percent deviation is seen. In principle this small effect would be useful, but $A_{\text {central }}^{\tilde{\chi}^{ \pm}}$, like $A_{F B}^{\tilde{\chi}^{ \pm}}$, is not directly observable and must be estimated through its correlation with some observable quantity. Even were this somehow to be overcome through Monte Carlo simulations, the chargino cross section at LEP II provides us with at most a few thousand events; statistical errors

on the measured $\sigma_{\text {total }}$ and $A_{F B}^{\tilde{\chi}^{ \pm}}$are already several percent. We therefore cannot expect to gain much from this variable.

We have searched for other possible observables, but have found none with both large variation and small experimental uncertainties; the behavior of $A_{\text {central }}^{\tilde{\chi}^{ \pm}}$is typical. Statistical errors alone make any of these variables difficult to use; but the impossibility of directly measuring the chargino momentum axis greatly complicates the determination of any variable based on distributions in chargino production angle. For our purposes, then, the total cross section and the forward-backward chargino production asymmetry are the only potentially useful quantities stemming from the unpolarized differential cross section. In the next subsections we discuss these two quantities in detail.

\section{Total Cross Section}

In this subsection we analyze the total cross section in detail. We will find that, as has been noted previously in the literature [2, 3, 4, 5, 6, 6, 8, 8, 9, 10, 21, 22, 29], charginos are produced in the thousands in most areas of the accessible parameter space, and the production cross section is highly sensitive to sneutrino mass.

From Eqs. (30) and (31) it can be seen that in the gaugino region, for small $\xi$, the cross section is approximately

$$
\begin{aligned}
\sigma_{\text {total }} & \approx f(v)\left\{1+\left[\frac{s}{s-M_{Z}^{2}}\right]^{2}\left(\frac{1}{4 \sin ^{2} \theta_{W}}\right)^{2}-\frac{\left|V_{11}\right|^{2}}{4 \sin ^{2} \theta_{W}}\left[1+\frac{s}{s-M_{Z}^{2}} \frac{1}{4 \sin ^{2} \theta_{W}}\right] \xi\right\} \\
& \approx(3.2-2.8 \xi) f(v) \mathrm{R} \approx(8.8 \mathrm{pb})(1-0.9 \xi) f(v)
\end{aligned}
$$

while in the Higgsino region it is

$$
\begin{aligned}
\sigma_{\text {total }} & \approx f(v)\left\{1+\left[\frac{s}{s-M_{Z}^{2}}\right]^{2}\left(\frac{\frac{1}{2}-\sin ^{2} \theta_{W}}{1-\sin ^{2} \theta_{W}}\right)^{2}\right\} \\
& \approx 1.3 f(v) \mathrm{R} \approx(3.6 \mathrm{pb}) f(v),
\end{aligned}
$$


where $f(v)=\frac{1}{2} v\left(3-v^{2}\right)$ rises from zero at threshold to one at high energy, $\xi$ is defined in Eq. (28), and where we have taken $\sqrt{s}=190 \mathrm{GeV}$, for which one unit of $\mathrm{R}$ is approximately $2.75 \mathrm{pb}$. Strong sensitivity to $m_{\tilde{\nu}}$ is found in the gaugino region [4,5,9], but disappears altogether in the Higgsino region. In the large $m_{\tilde{\nu}}$ limit both expressions are entirely determined by gauge invariance, but the event rate is two and a half times higher in the gaugino region. Notice that while a large cross section is a signal of the gaugino region, a small one can occur both in the Higgsino region and, if the sneutrino is light, in the gaugino region.

These features can all be seen in Fig. 9, where chargino production cross sections for $m_{\tilde{\nu}}=1 \mathrm{TeV}$ and $150 \mathrm{GeV}$ are plotted in picobarns. Because the cross section plots do not change substantially for different $\tan \beta$, the plots are presented for $\tan \beta=4$ only. We see that, in a sample of $1 \mathrm{fb}^{-1}$, LEP II will produce thousands of chargino events in most of the accessible regions of parameter space. Contrasting Figs. 9a and b, one can also see the strong dependence on $m_{\tilde{\nu}}$ noted above. This is shown more explicitly in Fig. 10, where we plot the total cross section at four representative $\left(\mu, M_{2}\right)$ points as a function of $m_{\tilde{\nu}}$. Clearly the $\tilde{\nu}$ diagram can give a large and destructive contribution to the cross section. For this reason, the infinite sneutrino mass limit is neither representative nor conservative and can lead to a substantial overestimate of the event rate in the gaugino region. This in turn could result in overly optimistic claims concerning the statistical accuracy with which SUSY parameters can be determined.

Having analyzed the dependence of $\sigma_{\text {total }}$ on the fundamental SUSY parameters, we now turn to the issue of how $\sigma_{\text {total }}$ may be extracted from experiment. To measure $\sigma_{\text {total }}$, it will be necessary to measure the partial cross sections of chargino production in at least two of the hadronic, mixed, and leptonic decay modes. The partial cross sections are given by

$$
\begin{aligned}
\sigma_{\text {leptonic }} & =B_{l}^{2} \sigma_{\text {total }} \\
\sigma_{\text {mixed }} & =2 B_{l}\left(1-B_{l}\right) \sigma_{\text {total }} \\
\sigma_{\text {hadronic }} & =\left(1-B_{l}\right)^{2} \sigma_{\text {total }}
\end{aligned}
$$

where $B_{l}$ is the leptonic branching fraction defined in Eq. (19). As these three partial cross sections depend on only two variables, they must satisfy the constraint

$$
\sigma_{\text {leptonic }} \sigma_{\text {hadronic }}=\frac{1}{4} \sigma_{\text {mixed }}^{2} \text {. }
$$

This relation is not dependent on the details of the chargino decay process; if it is not satisfied, it indicates a problem with the estimated detection efficiencies in one or more of the modes. (Such a discrepancy could stem either from an experimental problem or from physics beyond our minimal assumptions that is not included in the Monte Carlo simulation - for example, signal from an additional and unexpected supersymmetric particle.) To determine $\sigma_{\text {total }}$ it is best to use all three partial cross sections, subject to the constraint in Eq. 38, but we will only use the two with the smallest errors ( $\sigma_{\text {mixed }}$ and one of the other two). We note that in measuring $\sigma_{\text {total }}$, we obtain also $B_{l}$, and it is therefore natural to examine this observable, as we will do in Sec. IIIF.

\section{Chargino Forward-Backward Asymmetry}

The forward-backward asymmetry $A_{F B}^{\tilde{\chi}^{ \pm}}$of chargino production is theoretically attractive, since it can be computed analytically and depends on the four parameters $\left(\mu, M_{2}, \tan \beta, m_{\tilde{\nu}}\right)$ 
in a way which is quite different from the total cross section. In the large $m_{\tilde{\nu}}$ limit, $A_{F B}^{\tilde{\chi}^{ \pm}}$is negative in the mixed region and negligible elsewhere, as can be seen from Eqs. (30) and (32); however, a light sneutrino, which appears in a $t$-channel diagram, can give a large positive contribution to $A_{F B}^{\tilde{\chi}^{ \pm}}$in the gaugino and mixed regions. This effect is seen in Fig. 11.

Unfortunately, $A_{F B}^{\tilde{\chi}^{ \pm}}$cannot be directly measured; the velocities of the two charginos cannot be reconstructed because the two LSPs are invisible. Let us consider what might be possible to observe in these events. Since the forward-backward asymmetry is odd under charge conjugation, we must discover which chargino is positively charged. The sign of the charge of a single chargino can be determined in a leptonic decay from the charge of the lepton, but is more difficult to measure in a hadronic decay. We must also determine the momentum axis of the chargino. The visible particles in the decay of a chargino can in certain cases indicate the chargino momentum. For example, if the chargino is moving at relativistic velocities, the decay products are highly boosted along the chargino momentum axis. Alternatively, if in the chargino rest frame the total visible momentum, averaged over many events, is distributed isotropically, then the average visible momentum in any frame will give the chargino momentum direction. Unfortunately, neither of these cases applies here; the velocity of the chargino is generally semi- or non-relativistic, and the decays are often far from isotropic.

Still, we might hope that the distribution of the dijet momenta in the hadronic decays of charginos would give a reasonable estimate of the chargino momentum distribution and could be used to measure $A_{F B}^{\tilde{\chi}^{ \pm}}$. Specifically, in mixed mode chargino decays, we may use the dijet momentum to estimate the chargino momentum axis, and the charge of the lepton to determine the direction of the positively charged chargino. The dijet forward-backward asymmetry $A_{F B}^{j j}$ found in this way might well be correlated with $A_{F B}^{\tilde{\chi}^{ \pm}}$. Unfortunately, the correlation is often very weak. As described in Sec. \, for each case study, we have explored via Monte Carlo simulation the region of SUSY parameter space which gives the observed $m_{\tilde{\chi}_{1}^{ \pm}}, m_{\tilde{\chi}_{1}^{0}}, \sigma_{\text {total }}$ and $B_{l}$. We have found that for these points in parameter space it is impossible to estimate $A_{F B}^{\tilde{\chi}^{ \pm}}$using $A_{F B}^{j j}$ without additional information about the decay process. In fact, the variation of $A_{F B}^{j j}$ around $A_{F B}^{\tilde{\chi}^{ \pm}}$is so large that it indicates that $A_{F B}^{j j}$ has strong dependence on the parameters in the decay vertices, and possibly can be used to determine them. However, as analytic formulae for this variable are unavailable, and since experimental cuts must be included, this will require a detailed Monte Carlo simulation covering all of the allowed parameter space, which we do not attempt here. (We note that analytic formulae may be found near the threshold of chargino production, which may permit the separation of $A_{F B}^{\tilde{\chi}^{ \pm}}$from the decay vertices [39].)

Additionally, we have considered a range of cuts on the data to try to improve the correlation between $A_{F B}^{j j}$ and $A_{F B}^{\tilde{\chi}^{ \pm}}$. In particular, the angle between the dijet and chargino momenta may be determined simply from momentum conservation. By cutting away events where this angle is large, we obtain a sample of events where the dijet and chargino momenta point in roughly the same direction. In some cases the correlation is slightly improved for this sample, but this is counterbalanced by the loss of statistics. We note that this sample of events might be useful for other purposes, such as determining the parameters in the decay vertices.

We find, then, that it is impossible to measure $A_{F B}^{\tilde{\chi}^{ \pm}}$directly. However, the observable 
$A_{F B}^{j j}$, although too complicated to explore without a Monte Carlo simulation, contains a

mixture of information about $A_{F B}^{\tilde{\chi}^{ \pm}}$and the decay vertices that will certainly be of interest. We will discuss this further in Sec. $\nabla$.

\section{E. Polarization Asymmetry}

While the unpolarized cross section essentially contains only two separable parameters, a third independent observable,

$$
\frac{\sigma_{R}}{\sigma_{L}} \equiv \frac{\sigma\left(e_{R}^{-} e_{L}^{+} \rightarrow \tilde{\chi}^{+} \tilde{\chi}^{-}\right)}{\sigma\left(e_{L}^{-} e_{R}^{+} \rightarrow \tilde{\chi}^{+} \tilde{\chi}^{-}\right)},
$$

can be studied when polarized electron beams are available. Unfortunately, this is not expected to be the case at LEP II. Still, this observable has important implications for chargino threshold studies.

The ratio $\sigma_{R} / \sigma_{L}$ is less than $15 \%$ throughout the allowed region of parameter space, and so, even with unpolarized beams, charginos are always produced largely by left-handed electrons [3,28,29]. In the large $m_{\tilde{\nu}}$ limit, $\sigma_{R} / \sigma_{L} \sim 1 \%$ in the gaugino and $\sim 15 \%$ in the Higgsino region; it varies widely in the mixed region, but is generally small. A light sneutrino can increase the ratio in the gaugino and mixed region. In Fig. 12 we show $\sigma_{R} / \sigma_{L}$ for $m_{\tilde{\nu}}=150 \mathrm{GeV}$, for which the ratio takes values near its maximum. Nevertheless, we see that $\sigma_{R} / \sigma_{L}$ is still small, and is approximately $2 \%$ in much of the gaugino region. While this ratio is independent of $\sigma_{\text {total }}$ and $A_{F B}^{\tilde{\chi}^{ \pm}}$, it is not uncorrelated with the total cross section $\sigma_{\text {total }}=\sigma_{R}+\sigma_{L}$, since $m_{\tilde{\nu}}$ enters only in $\sigma_{L}$ and $\sigma_{L} \gg \sigma_{R}$. Still, the correlation is imperfect and it is possible to gain some amount of new information.

It is possible to exploit experimentally the theoretical prediction that $\sigma_{R} / \sigma_{L}$ is small. Near threshold, this implies that charginos are produced dominantly polarized along the beam axis, and the study of their decays is therefore greatly simplified. As the combinations of the SUSY parameters that enter the decay are different from those that enter the production process, measurements at threshold can give valuable information to supplement the analysis presented in this paper [39]. In this study we assume a fixed center-of-mass energy, which means that in much of the parameter region the charginos have substantial velocities, and near-threshold analysis is not applicable.

\section{F. Leptonic Branching Fraction}

To measure the total cross section, we must determine at least two of the partial cross sections for the hadronic, mixed, and leptonic modes. These also provide us with a measurement of the leptonic branching fraction $B_{l}\left(\mu, M_{2}, \tan \beta, M_{1}, m_{\tilde{l}}, m_{\tilde{q}}\right)$, which can vary greatly in the SUSY parameter space [40]. We now analyze the dependence of $B_{l}$ on the underlying SUSY parameters.

As discussed earlier, charginos decay to a neutralino and either two hadrons or two leptons. The hadronic decays occur via intermediate $W$ bosons and squarks (Fig. 2). The decay $\tilde{\chi}_{1}^{+} \rightarrow \tilde{\chi}_{1}^{0} u \bar{d}$ through a virtual $W$ has amplitude 


$$
\bar{u}\left(\tilde{\chi}_{1}^{0}\right) i g \gamma^{\mu}\left(O_{L} P_{L}+O_{R} P_{R}\right) u\left(\tilde{\chi}_{1}^{+}\right) \times \bar{u}(u) \frac{-i g}{\sqrt{2}} \gamma_{\mu} P_{L} v(\bar{d}) \times \frac{-i}{\left[\left(p_{u}+p_{\bar{d}}\right)^{2}-M_{W}^{2}\right]},
$$

where

$$
\begin{aligned}
& O_{L} \equiv \mathbf{N}_{12} \mathbf{V}_{11}^{*}-\frac{1}{\sqrt{2}} \mathbf{N}_{14} \mathbf{V}_{12}^{*} \\
& O_{R} \equiv \mathbf{N}_{12}^{*} \mathbf{U}_{11}+\frac{1}{\sqrt{2}} \mathbf{N}_{13}^{*} \mathbf{U}_{12} .
\end{aligned}
$$

The decay mediated by a left-handed up squark has amplitude

$$
\begin{aligned}
\bar{v}\left(\tilde{\chi}_{1}^{+}\right)(-i g) & \mathbf{V}_{11}^{*} P_{L} v(\bar{d}) \\
& \times \bar{u}(u)(-i g \sqrt{2})\left(Y_{Q} \tan \theta_{W} \mathbf{N}_{11}+I_{u} \mathbf{N}_{12}\right) P_{R} v\left(\tilde{\chi}_{1}^{0}\right) \frac{i}{\left[\left(p_{\tilde{\chi}^{0}}+p_{u}\right)^{2}-m_{\tilde{u}_{L}}^{2}\right]},
\end{aligned}
$$

while that mediated by a left-handed down squark takes the form

$$
\begin{aligned}
-\bar{u}(u)(-i g) & \mathbf{U}_{11} P_{R} u\left(\tilde{\chi}_{1}^{+}\right) \\
& \times \bar{u}\left(\tilde{\chi}_{1}^{0}\right)(-i g \sqrt{2})\left(Y_{Q} \tan \theta_{W} \mathbf{N}_{11}^{*}+I_{d} \mathbf{N}_{12}^{*}\right) P_{L} v(\bar{d}) \times \frac{i}{\left[\left(p_{\tilde{\chi}^{0}}+p_{\bar{d}}\right)^{2}-m_{\tilde{d}_{L}}^{2}\right]} .
\end{aligned}
$$

The overall minus sign of the down squark amplitude results from the odd permutation of the spinors of the down squark amplitude relative to the spinors of the other two diagrams. In these formulae $Y_{Q}=\frac{1}{6}$ is the hypercharge of left-handed quarks, while $I_{u}\left(I_{d}\right)=\frac{1}{2}\left(-\frac{1}{2}\right)$ is the weak isospin of up (down) quarks. We remind the reader that only the first two generations of squarks participate in chargino decays, since we have assumed that squark mixing angles are small and that the chargino is lighter than the top quark.

In Eqs. (42) and (43) we have omitted the couplings of squarks to the Higgsino component of the gauginos, because they are suppressed by $m_{d} /\left(M_{W} \cos \beta\right)$ or $m_{u} /\left(M_{W} \sin \beta\right)$, and are therefore negligible. As discussed in Sec. [1], the right-handed squark diagrams are similarly suppressed and may also be ignored. (We note, however, that the very small effects of the Higgsino couplings and the right-handed sfermion diagrams are included in our Monte Carlo simulation.)

The squark diagram contributions may be Fierz transformed into the same form as the $W$ diagram amplitude; the full hadronic decay amplitude may then be written as

$$
\frac{i^{3} g^{2}}{\sqrt{2}} \bar{u}\left(\tilde{\chi}_{1}^{0}\right) \gamma^{\mu}\left(D_{L} P_{L}+D_{R} P_{R}\right) u\left(\tilde{\chi}_{1}^{+}\right) \times \bar{u}(u) \gamma_{\mu} P_{L} v(\bar{d})
$$

where

$$
D_{L}(q)=\frac{O_{L}}{\left(p_{u}+p_{\bar{d}}\right)^{2}-M_{W}^{2}}+\frac{\mathbf{V}_{11}^{*}\left(\frac{1}{6} \tan \theta_{W} \mathbf{N}_{11}+\frac{1}{2} \mathbf{N}_{12}\right)}{\left(p_{\tilde{\chi}_{1}^{0}}+p_{u}\right)^{2}-m_{\tilde{u}_{L}}^{2}}
$$

and

$$
D_{R}(q)=\frac{O_{R}}{\left(p_{u}+p_{\bar{d}}\right)^{2}-M_{W}^{2}}-\frac{\mathbf{U}_{11}\left(\frac{1}{6} \tan \theta_{W} \mathbf{N}_{11}^{*}-\frac{1}{2} \mathbf{N}_{12}^{*}\right)}{\left(p_{\tilde{\chi}_{1}^{0}}+p_{\bar{d}}\right)^{2}-m_{\tilde{d}_{L}}^{2}}
$$

Notice that up(down)-type squarks contribute only to $D_{L}\left(D_{R}\right)$. 
For leptonic decays, in which all three generations of sleptons participate, the full amplitude has the same form as Eq. (44) but with hypercharge equal to $-\frac{1}{2}$ :

$$
D_{L}(l)=\frac{O_{L}}{\left(p_{\nu}+p_{\bar{e}}\right)^{2}-M_{W}^{2}}+\frac{\mathbf{V}_{11}^{*}\left(-\frac{1}{2} \tan \theta_{W} \mathbf{N}_{11}+\frac{1}{2} \mathbf{N}_{12}\right)}{\left(p_{\tilde{\chi}_{1}^{0}}+p_{\nu}\right)^{2}-m_{\tilde{\nu}_{L}}^{2}},
$$

and

$$
D_{R}(l)=\frac{O_{R}}{\left(p_{\nu}+p_{\bar{e}}\right)^{2}-M_{W}^{2}}-\frac{\mathbf{U}_{11}\left(-\frac{1}{2} \tan \theta_{W} \mathbf{N}_{11}^{*}-\frac{1}{2} \mathbf{N}_{12}^{*}\right)}{\left(p_{\tilde{\chi}_{1}^{0}}+p_{\bar{e}}\right)^{2}-m_{\tilde{e}_{L}}^{2}} .
$$

As above, the isospin $+\frac{1}{2}\left(-\frac{1}{2}\right)$ left-handed slepton contributes to $D_{L}\left(D_{R}\right)$.

In the approximation that the momentum dependences of the $W$ and scalar propagators are ignored, the partial widths of the chargino can be written down in closed form. In this limit, the width, in terms of $r=m_{\tilde{\chi}_{1}^{0}} / m_{\tilde{\chi}_{1}^{ \pm}}$, is

$$
\Gamma\left(\tilde{\chi}^{+} \rightarrow \tilde{\chi}^{0} f \bar{f}\right) \approx N_{f} N_{c} \frac{g^{4} m_{\tilde{\chi}_{1}^{ \pm}}^{5}}{3072 \pi^{3}} G(r)\left[D_{L}(f)^{2}+D_{R}(f)^{2}-g(r) D_{L}(f) D_{R}(f)\right],
$$

where

$$
\begin{aligned}
G(r) & =1-8 r^{2}-24 r^{4} \log r+8 r^{6}-r^{8} \\
g(r) & =4 r\left(1+9 r^{2}+12 r^{2} \log r-9 r^{4}+12 r^{4} \log (r)-r^{6}\right) / G(r),
\end{aligned}
$$

and where for hadrons (leptons), $f=q(f=l)$, the number of flavors $N_{f}$ is $2(3)$, and the number of colors $N_{c}$ is $3(1)$. The function $g(r)$ is well approximated by $1-(1-r)^{4}$, while for $r \rightarrow 1, G(r) \sim(1-r)^{5}$. The ratio of leptonic to hadronic branching fractions is given by

$$
\frac{B_{l}}{1-B_{l}}=\frac{1}{2} \frac{D_{L}^{2}(l)+D_{R}^{2}(l)-g(r) D_{L}(l) D_{R}(l)}{D_{L}^{2}(q)+D_{R}^{2}(q)-g(r) D_{L}(q) D_{R}(q)} .
$$

The dependence of $B_{l}$ on the parameters of supersymmetry is quite complicated, and there are few regions of parameter space in which a useful perturbative expansion may be performed. However, it is possible to make some broad statements about its behavior. In Fig. 13 we present $B_{l}$ for three different values of the parameters. Note that $B_{l}$ is unlike the other three variables we have looked at, in that it can have strong $\tan \beta$ dependence in the gaugino region. In the following we will discuss some of the most notable features in the figures.

In the Higgsino region of Figs. $13 \mathrm{a}$-c , the branching fraction is approximately $\frac{1}{3}$. This is a general phenomenon. As the Higgsino region is approached from the gaugino region, the couplings of the chargino to squarks and sleptons decrease, while the couplings to the $W$ boson increase. In the far Higgsino region, the sfermion couplings to the chargino are completely negligible, so chargino decay is dominated by virtual $W$ bosons for which the branching ratio $B_{l}$ is $\frac{1}{3}$. (Recall that we include in $B_{l}$ all chargino decays to $\tau$ leptons, even if the $\tau$ itself decays hadronically.) Even outside the Higgsino region, effects of heavy squarks and sleptons are suppressed relative to the intermediate $W$ boson; the large sfermion mass limit again leads to $B_{l} \approx \frac{1}{3}$. Thus, we cannot draw any conclusions if $B_{l} \approx \frac{1}{3}$, but if $B_{l}$ is not equal to $\frac{1}{3}$, it immediately rules out both the Higgsino region and ultraheavy sfermions. 
In the gaugino region, where $|\mu| \gg M_{2}$, the matrix elements $\mathbf{U}_{11}, \mathbf{V}_{11}$ and $\mathbf{N}_{11}$ are all very close to unity, while the couplings $O_{L}$ and $O_{R}$ of the chargino to the $W$ are of order the mixing angle $\mathbf{N}_{12}$, as are the isospin-dependent terms in $D_{L, R}(l, q)$. All other relevant elements of the $\mathbf{U}, \mathbf{V}$ and $\mathbf{N}$ matrices are small. A perturbative diagonalization of the matrix in Eq. (6) shows that in the range of parameters we consider,

$$
\mathbf{N}_{12} \sim\left(\frac{M_{Z}}{\mu}\right)^{n},
$$

where $n=1$ for $M_{Z} \tan \beta \ll|\mu|$, and $n=2$ for $M_{Z} \tan \beta \gg|\mu|$. The latter reflects the $\mu \rightarrow-\mu$ symmetry for large $\tan \beta$.

It follows that for very large $|\mu|$ and sufficiently small slepton and squark masses, the $W$ diagram and the isospin terms in the squark and the slepton diagrams are negligible, as can be seen from Eqs. (42) and (43). Keeping only the hypercharge terms in $D_{L, R}(q, l)$ and noting $\left(Y_{l} / Y_{q}\right)^{2}=9$, one finds

$$
B_{l} \approx \frac{m_{\tilde{q}}^{4}}{m_{\tilde{q}}^{4}+\frac{2}{9} m_{\tilde{l}}^{4}}
$$

in the far gaugino region. This is larger than $\frac{1}{3}$ for each of the cases plotted in Fig. 13, in which the growth of $B_{l}$ at large $|\mu|$ is evident. Notice, however, that the growth is much faster in the large $\tan \beta$ case (Fig. [13k) than in the small $\tan \beta$ case (Figs. [13a,b). Note also that the figures at $\tan \beta=2$ with two different squark masses (Figs. 13a, b) have $B_{l}$ contours that are shifted by a constant factor. We explain these features below.

It is possible to estimate roughly where the crossover occurs from isospin dominated chargino decays, which are close to $B_{l}=\frac{1}{3}$ for $m_{\tilde{q}} \sim m_{\tilde{l}}$, to hypercharge dominated decays, which have a $B_{l}$ given by Eq. (53). We first consider small $\tan \beta$. Take $|\mu| \gg M_{Z} \tan \beta$, but in the near gaugino region, where $B_{l} \sim \frac{1}{3}$. Now raise $|\mu|$ until $\mathbf{N}_{12}$ is so small that the hypercharge terms in Eqs. (42) and (43) dominate the decay amplitudes. Since, for small $\tan \beta, \mathbf{N}_{12} \sim M_{Z} / \mu$, we may estimate that this occurs for

$$
|\mu| \sim \frac{1}{\tan \theta_{W}\left|Y_{f}\right|} \frac{m_{\tilde{f}}^{2}}{M_{W}^{2}} M_{Z} .
$$

This rough estimate is accurate only within a factor of four or so. Since $\tan \theta_{W}\left|Y_{l}\right|$ is approximately $1 / 4$ for leptons and $1 / 12$ for quarks, the required $|\mu|$ is generally larger than a $\mathrm{TeV}$ except for the lightest sfermions, and is smaller for sleptons than for squarks of the same mass. We therefore expect that for small $\tan \beta, B_{l}$ will not stray too far from $\frac{1}{3}$. In the limit $\tan \beta=1$ we may quantify this; for large squark and slepton masses, the leading hypercharge terms in the $D_{L, R}(q, l)$ cancel, with the isospin terms giving

$$
B_{l} \approx \frac{1}{3}\left[1+\frac{2}{3}\left(\frac{M_{W}^{2}}{m_{\tilde{l}}^{2}}-\frac{M_{W}^{2}}{m_{\tilde{q}}^{2}}\right)\right],
$$

to first order in $M_{W}^{2} / m_{\tilde{l}}^{2}$ and $M_{W}^{2} / m_{\tilde{q}}^{2}$. This shows that even within the isospin terms there is sensitivity, independent of $\sin \theta_{W}$ and $\tan \beta$, to the difference between $m_{\tilde{q}}$ and $m_{\tilde{l}}$. This 
is a general feature of small $\tan \beta$, as is reflected in Figs. 13a and $\mathrm{b}$, where the $B_{l}$ contours differ by approximately $5 \%$ and are consistent with Eq. (55).

Suppose instead that $\tan \beta$ is large and take $|\mu| \ll M_{Z} \tan \beta$, again in the near gaugino region with $B_{l} \sim \frac{1}{3}$. Let us again consider increasing $|\mu|$. Now $\mathbf{N}_{12} \sim\left(M_{Z} / \mu\right)^{2}$ vanishes much more quickly as $|\mu|$ grows; the crossover to Eq. (53) occurs near

$$
|\mu| \sim \frac{1}{\sqrt{\tan \theta_{W}\left|Y_{f}\right|}} \frac{m_{\tilde{f}}}{M_{W}} M_{Z}
$$

The dependence on the sfermion mass is now linear, and the coefficient has become smaller as well. For large $\tan \beta$, the leptonic branching fraction will therefore deviate strongly from

$\frac{1}{3}$ at much smaller values of $|\mu|$ than is the case for small $\tan \beta$. This is clearly seen in the difference between Figs. 13a and c. We note that in the near gaugino region, $B_{l}$ is also shifted by the correction term in Eq. (55) even for large $\tan \beta$.

Another feature worth noting is a "pocket" in which $B_{l}$ drops below $\frac{1}{3}$, which occurs in the near gaugino region for negative $\mu$, where simple perturbation theory is not applicable. In this region, the slepton diagrams interfere destructively with the $W$ diagram in the decay amplitude. The "pocket" can be deep for intermediate values of $\tan \beta$, where $B_{l}$ can take values as low as $10 \%$. This feature has important implications for the case studies of Sec. D.

As noted above, $B_{l}$ can be determined by measuring the partial cross sections. The leptonic branching fraction is given by

$$
B_{l}=\frac{2 \sigma_{\text {leptonic }}}{2 \sigma_{\text {leptonic }}+\sigma_{\text {mixed }}}=\frac{\sigma_{\text {mixed }}}{\sigma_{\text {mixed }}+2 \sigma_{\text {hadronic }}} .
$$

Again, all three partial cross sections should be employed, though we will only use the two that are likely to have the smallest errors for our measurement of $B_{l}$. Strictly speaking, the partial cross sections must be adjusted to account for the fact that $\tau$ leptons can decay hadronically. As the corrections depend solely on the well-measured $\tau$ branching fractions, the required adjustments are very reliable.

\section{G. Other Observables}

There are many other quantities that could be extracted from the data which depend on the details of the chargino decay vertices. For $2 j+l$ events, distributions of $\theta_{l}$, the angle between the lepton and the beam axis, $E_{l}$, the energy of the lepton, and corresponding dijet variables $\theta_{j j}$ and $E_{j j}$, are observables that are likely to yield useful information. Correlations between $l$ and $\bar{l}$ angles in $2 l$ events and between lepton and dijet angles in $2 j+l$ may also provide information [41]. However, to obtain analytic expressions for these variables one would have to convolve the angular velocity and spin distribution of the production process with the three-body spin-dependent phase space of the decay. The complexity of dealing with these observables is regrettable, since the angular distributions of the chargino decay products are sensitive to $D_{L}(q) / D_{R}(q)$ and $D_{L}(l) / D_{R}(l)$ (see Sec. IIIF), which cannot be probed using the quantities $m_{\tilde{\chi}_{1}^{ \pm}}, m_{\tilde{\chi}_{1}^{0}}, \sigma_{\text {total }}$ and $B_{l}$. Furthermore, the angular distributions might help determine the angle and spin dependence of the differential cross section, and might be noticeably affected by the propagators of very light sfermions. Restrictions on 
SUSY parameter space using these observables will probably require detailed Monte Carlo simulations, which we will not attempt to carry out. It seems plausible to us that a global maximum likelihood fit to the data, on the basis of a sufficiently thorough Monte Carlo search, should be able to pick out information that we have not been able to extract in our analysis; we will bolster this claim in Sec. $\nabla$. It remains to be seen whether the additional knowledge will lead to significantly improved constraints on the underlying parameters.

There are other variables worthy of study which allow the assumptions of our analysis to be tested. If the LSP is a sneutrino instead of a neutralino, chargino decays are qualitatively different, since the two-body decay $\tilde{\chi}_{1}^{ \pm} \rightarrow l \tilde{\nu}$ becomes available. If there is substantial mixing between any of the right- and left-handed sfermions, the chargino decay amplitude will no longer have the form of Eq. (44); the resulting angular distribution of the observed fermions will be identifiably different. Mixing of the third generation of squarks into the other generations could cause many $b$ quarks to appear in the dijets; under our assumptions, very few are expected. A significant breakdown of the universal slepton mass assumption would affect lepton universality in the decays; similarly, a violation of our universal squark mass assumption for the first two generations might be detected by studying the abundance of charm quarks in the dijets. Lastly, a significant contribution by intermediate charged Higgs bosons to the chargino decay amplitude would both affect the angular distributions of the observed fermions and lead to extra heavy fermions among the decay products.

\section{H. Summary of Observables}

We have now concluded the discussion of the four primary observables that will be used in the case studies below. These observables, with their dependences on the underlying SUSY parameters, are the chargino mass $m_{\tilde{\chi}_{1}^{ \pm}}\left(\mu, M_{2}, \tan \beta\right)$, the LSP mass $m_{\tilde{\chi}_{1}^{0}}\left(\mu, M_{2}, \tan \beta, M_{1}\right)$, the total cross section $\sigma_{\text {total }}\left(\mu, M_{2}, \tan \beta, m_{\tilde{l}}=m_{\tilde{\nu}}\right)$, and the leptonic branching fraction $B_{l}\left(\mu, M_{2}, \tan \beta, M_{1}, m_{\tilde{l}}, m_{\tilde{q}}\right)$. We have also studied the forward-backward asymmetry $A_{F B}^{\tilde{\chi}^{ \pm}}\left(\mu, M_{2}, \tan \beta, m_{\tilde{l}}=m_{\tilde{\nu}}\right)$ and have explained why it is difficult to use.

To close this section, we review the sensitivity of these parameters in the different regions. In the gaugino region, where $|\mu| \gg M_{2},\left|M_{1}\right|$, we are sensitive to all six parameters: $m_{\tilde{\chi}_{1}^{ \pm}} \sim$ $M_{2} ; m_{\tilde{\chi}_{1}^{0}} \sim\left|M_{1}\right| ; \sigma_{\text {total }} \approx f(v) \times\left[3.2-2.8\left(s / m_{\tilde{\nu}}^{2}\right)\right]$ R to leading order in $s / m_{\tilde{\nu}}^{2}$, where $f(v)$ is a definite function of the chargino velocity; and $B_{l}$ is a non-trivial function which is sensitive to several combinations of SUSY parameters. When $m_{\tilde{l}}\left(=m_{\tilde{\nu}}\right)$ and $m_{\tilde{q}}$ are both very large, then $\sigma_{\text {total }}=f(v) \times 3.2 \mathrm{R}$ and $B_{l}=\frac{1}{3}$. In the Higgsino region, where $|\mu| \ll M_{2}$, the following relationships hold: $m_{\tilde{\chi}_{1}^{ \pm}} \sim|\mu| ; m_{\tilde{\chi}_{1}^{0}} \sim \min \left\{|\mu|,\left|M_{1}\right|\right\} ; \sigma_{\text {total }}=f(v) \times 1.3 \mathrm{R}$; and $B_{l}=\frac{1}{3}$. There is no sensitivity in this region to $m_{\tilde{l}}$ or $m_{\tilde{q}}$, and very little to $M_{1} / M_{2}$, unless $\left|M_{1}\right|$ is less than or of order $|\mu|$. In the mixed region, the observables are all complicated functions of the SUSY parameters, and there are few general statements to be made.

From this discussion it can be guessed that we can put the fewest constraints on parameter space if the physics lies in the Higgsino region, whereas the gaugino region is more promising for our analysis. Intermediate results are found in the mixed region. We will see this explicitly in the case studies in Sec. V. 


\section{EVENT SIMULATION AND BACKGROUNDS}

For this study, chargino events are generated by a simple parton level Monte Carlo event generator. For a given set of parameters, we first calculate the chargino decay width and branching ratios. The SAGE subroutines [42] are used to generate three-body final state momenta and phase space weightings, and the matrix elements for the decay are calculated with the explicit helicity spinor method, using subroutines that are patterned after the HELAS package [43. We find that typically the chargino width $\Gamma_{\tilde{\chi}_{1}^{ \pm}}$is roughly $1-100 \mathrm{keV}$. Using the same subroutines, we therefore generate the six-body events $e^{+} e^{-} \rightarrow \tilde{\chi}_{1}^{+} \tilde{\chi}_{1}^{-} \rightarrow$ $\left(\tilde{\chi}_{1}^{0} q^{\prime} q, \tilde{\chi}_{1}^{0} l \nu\right)\left(\tilde{\chi}_{1}^{0} q^{\prime} q, \tilde{\chi}_{1}^{0} l \nu\right)$ in the zero width approximation for the intermediate charginos. In this approximation the total amplitude factorizes into production and decay amplitudes and is given by

$$
\mathcal{M}^{\text {tot }}=\sum_{h^{+}, h^{-}=-1}^{1} \mathcal{M}_{h^{+} h^{-}}^{\text {prod }} \mathcal{M}_{h^{+}}^{\text {decay }} \mathcal{M}_{h^{-}}^{\text {decay }} \frac{\pi}{m_{\tilde{\chi}_{1}^{ \pm}} \Gamma_{\tilde{\chi}_{1}^{ \pm}}}
$$

where

$$
\begin{aligned}
\mathcal{M}_{h^{+} h^{-}}^{\text {prod }} & =\mathcal{M}^{\text {prod }}\left(e^{+} e^{-} \rightarrow \tilde{\chi}_{h^{+}}^{+} \tilde{\chi}_{h^{-}}^{-}\right) \\
\mathcal{M}_{h^{ \pm}}^{\text {decay }} & =\mathcal{M}^{\text {decay }}\left(\tilde{\chi}_{h^{ \pm}}^{ \pm} \rightarrow \tilde{\chi}_{1}^{0} q^{\prime} q, \tilde{\chi}_{1}^{0} l \nu\right),
\end{aligned}
$$

and $h^{ \pm}$is the helicity of $\tilde{\chi}_{1}^{ \pm}$. Without factorization the amplitude consists of up to 108 diagrams, since 3 diagrams contribute to the production process and 6 (5) diagrams contribute to each hadronic (leptonic) decay. (Of course, with the assumptions of Sec. II, only 3 (3) diagrams contribute substantially to the hadronic (leptonic) decay.) Factorization allows us to calculate $\mathcal{M}_{h^{+} h^{-}}^{\text {prod }}$ and $\mathcal{M}_{h^{ \pm}}^{\text {decay }}$ separately, which is a great simplification and considerably improves the efficiency of the event generator. After calculating these amplitudes, we sum over internal chargino helicities to get $\mathcal{M}^{\text {tot }}$, which is then squared and summed over external helicities to obtain the total differential cross section. By summing over internal helicities before squaring, we retain the important spin correlations between production and decay.

In this study, we ignore effects due to the Majorana nature of the $\tilde{\chi}_{1}^{0}$. Because the $\tilde{\chi}_{1}^{0}$ is a Majorana fermion, one should in principle include for every Feynman diagram a diagram with the $\tilde{\chi}_{1}^{0}$ momenta interchanged. In our Monte Carlo simulation, the momenta are preferentially picked such that the charginos are very nearly on-shell. The chargino rest frames are boosted with respect to each other, and most events are produced with the two LSPs having different momenta. Thus, in almost all cases, the diagram with interchanged LSP momenta has chargino momenta that are far out of their narrow Breit-Wigner peaks and can be ignored.

The event generator was checked in a number of ways. In the explicit spinor method, Lorentz invariance is not manifest. We have checked that the total amplitude squared is invariant under arbitrary Lorentz boosts, and this provides a powerful check. In addition, the amplitudes must transform into their complex conjugates, up to a sign, when all helicities are reversed, and this was verified as well. The production cross section was found to agree with the analytic results presented in Sec. [II] and with those previously published [5] for many sets of parameters. The decay amplitudes were also verified in a number of ways. The 
differential decay width $d \Gamma / d E d \cos \theta$ was found to agree with muon decay in the appropriate limit, and also with the decay of a "muon" with $\mathrm{V}+\mathrm{A}$ coupling. This provides a check of the $W$ diagram and the overall normalization. Finally, the total decay amplitude, summed over all diagrams, was verified to reproduce the chargino branching ratio results given in Ref. [40].

Effects of initial state radiation (ISR) are not included in our simulation. Hadronization and detector effects are crudely simulated by smearing quark parton and lepton energies. The detector resolutions currently available at the ALEPH detector at LEP are [12]

$$
\sigma_{E}^{\mathrm{had}} / E=80 \% / \sqrt{E} \quad \text { and } \quad \sigma_{E}^{\text {e.m. }} / E=19 \% / \sqrt{E},
$$

where $E$ is in $\mathrm{GeV}$. In the Monte Carlo simulation, we smear the lepton parton energies by the leptonic resolution. For the typical energies of our simulation, the resulting leptonic resolutions are numerically a good approximation to those that will be achieved for both muons and electrons by current LEP detectors. We also smear the hadronic parton energies by the hadronic resolution. By doing so, we implicitly assume that the quark jet energy is measured by the hadronic calorimeter only. In fact, however, quark jets are detected by a combination of the tracking chamber, the electromagnetic calorimeter, and the hadronic calorimeter, and, in particular, the addition of tracking chamber measurements can improve the jet energy resolution substantially. This improvement was studied in Ref. 44 for $W$ mass resolution at a $\sqrt{s}=500 \mathrm{GeV} e^{+} e^{-}$collider. For a detector with energy resolutions $\sigma_{E}^{\text {had }} / E=50 \% / \sqrt{E}+2 \%$ and $\sigma_{E}^{\text {e.m. }} / E=10 \% / \sqrt{E}+2 \%$, the resolution of $M_{W}$, as measured by the dijet mass, was found to improve by $33 \%$ from $4.1 \mathrm{GeV}$ to $2.7 \mathrm{GeV}$ when the tracking chamber measurements were included. Similar studies for the L3 detector at LEP have shown that when the momentum measurement from the central tracking detector is included, the resolution for the total energy in hadronic events improves by about $20 \%$ from $9.2 \mathrm{GeV}$ to 7.6 $\mathrm{GeV}$ [45]. To simply account for the improvements from tracking chamber measurements, we will reduce our resolutions for $m_{j j}$ and $E_{j j}$ endpoint determinations by $25 \%$ by hand in the case studies presented below.

To study the observables presented in Sec. III, we must find the $m_{j j}$ and $E_{j j}$ distributions in $2 j+l$ chargino events and determine two of the partial cross sections, including that of the mixed mode. Three of these measurements can be made with $Y$ mode events, the subset of $2 j+l$ events in which the hadrons do not come from a tau lepton, since the $Y$ mode cross section can be converted to a mixed mode cross section (under the assumption that lepton universality holds). It is therefore important to include realistic cuts that isolate the $Y$ mode chargino events. We will now show that $2 j+l$ events may be easily resolved. In Sec. $\mathrm{V}$ we will see that the $Y$ mode events in this sample may be isolated by simply eliminating events with low invariant hadronic mass.

The $2 j+l$ mode is the most promising for chargino discovery and has been carefully studied [6,7],8]. In Ref. [8] cuts have been designed for the parameters $\sqrt{s}=175 \mathrm{GeV}$, $\mu=-100 \mathrm{GeV}, \tan \beta=2, m_{\tilde{\chi}_{1}^{ \pm}}=80 \mathrm{GeV}$, and $m_{\tilde{\chi}_{1}^{0}}=20-60 \mathrm{GeV}$. These cuts include the following requirements:

1) The missing transverse momentum $\not \not_{T}$ is required to be greater than $10 \mathrm{GeV}$.

2) The event must contain an isolated electron or muon with a momentum larger than 5 $\mathrm{GeV}$ and with no hadronic activity within $30^{\circ}$.

3) The squared missing mass $m_{\text {missing }}^{2}$ must be greater than $4000 \mathrm{GeV}^{2}$. 
4) The hadronic system mass $m_{j j}$ must be less than $45 \mathrm{GeV}$.

5) Under the assumption that the missing momentum in the event is due to a "neutrino", the mass of the lepton- "neutrino" system is required to be less than $70 \mathrm{GeV}$. This removes most $W W$ events. Actually, one can do even better than this, since it is possible to allow for two unobserved massless particles, one an undetected ISR photon along the beam axis and the other a missing "neutrino", and still determine all of the momenta in the event. In this case, forcing the lepton- "neutrino" invariant mass to be less than $70 \mathrm{GeV}$ removes many $W W \gamma_{I S R}$ events as well.

As shown in Ref. [8], cuts 1 and 2 reduce the $W W$ background to $2.8 \mathrm{pb}$. Cuts $3-5$ are specifically designed to reduce this background further. After additionally imposing cuts 3 , 4, and 5, the $W W$ background is reduced to $180 \mathrm{fb}, 17 \mathrm{fb}$, and $7 \mathrm{fb}$, respectively. After all cuts, the other standard model backgrounds contribute only $2 \mathrm{fb}$. Applying these same cuts to a chargino sample, we have found that typically the mixed mode is reduced by about 25-75\% after cut 4 , but $40 \%$ of these are eliminated by cut 5 . Although the additional $40 \%$ loss in statistics is not extremely large, typically the signal to background ratio is greater than or of order 50 after cut 4 and the background is already negligible. Thus, of the five cuts listed, we will use cuts 1-4 and ignore cut 5 in our analysis. This leaves the standard model background at approximately $20 \mathrm{fb}$.

As we do not include the effects of hadronization, we will also require that each final state quark parton have energy greater than $5 \mathrm{GeV}$ so that its hadronization products are detected.

In addition to standard model backgrounds, there may be supersymmetric backgrounds that will need to be distinguished from chargino pairs. In particular, as noted in Sec. II, if charginos are produced, neutralino production is almost certainly allowed. It is first worth noting that the neutralino background is highly suppressed in a significant portion of parameter space [20]. Neutralinos are produced through a $Z$ annihilation diagram and $t$-channel selectron exchange. However, as the $Z$ couples only to the Higgsino components of the neutralinos, neutralino production is suppressed in the gaugino region unless the selectron mass is low. It may happen, then, that neutralino production, though kinematically allowed, is a negligible background to chargino production. For other regions of parameter space, it should still be possible to separate the neutralino and chargino events. Production of $\tilde{\chi}_{1}^{0} \tilde{\chi}_{2}^{0}$ is not a significant background to $Y$ mode chargino events, because these neutralino events produce exactly one isolated charged lepton only when $\tilde{\chi}_{2}^{0} \rightarrow \tilde{\chi}_{1}^{0} \tau \bar{\tau}$, and one $\tau$ decays leptonically and the other hadronically. These should be easily distinguished from $Y$ mode chargino events, based on the small invariant mass of the hadrons. Production of $\tilde{\chi}_{2}^{0} \tilde{\chi}_{2}^{0}$ may be problematic if one neutralino decays to $\tilde{\chi}_{1}^{0} \tau \bar{\tau}$ while the other decays to $\tilde{\chi}_{1}^{0} q \bar{q}$, which can lead to an isolated lepton and an assortment of hadrons. However, even if the hadrons cannot be resolved into three isolated jets, kinematics often distinguish these events from chargino events, since the total hadronic energy or invariant mass may exceed what is allowed in $\tilde{\chi}_{1}^{ \pm} \rightarrow \tilde{\chi}_{1}^{0} q \bar{q}$. In any case, the number of these events is usually very small. Both types of neutralino events may be backgrounds for the purely leptonic chargino events, and $\tilde{\chi}_{2}^{0} \tilde{\chi}_{2}^{0}$ may also be a background for the four-jet events. However, neutralino events do not produce significant numbers of $e \mu$ events, nor do they produce $\bar{l} l$ events with $m_{\bar{l} l}>m_{\tilde{\chi}_{2}^{0}}-m_{\tilde{\chi}_{1}^{0}}$, and these may therefore be identified as chargino events. Assuming lepton universality, one can then determine both the total leptonic and total hadronic cross section from chargino pairs. 
If cascade decays occur with a substantial branching fraction, they may also make it difficult to isolate the chargino signal. Such decays are prominent, for example, in certain regions with $M_{2} \sim|\mu| \sim M_{W}$, where the mass separation between the $\tilde{\chi}_{1}^{ \pm}$and the $\tilde{\chi}_{2}^{0}$ is large, and where $\tilde{\chi}_{1}^{0} \tilde{\chi}_{3}^{0}$ production may be possible. We assumed in Sec. II that cascade decays of the chargino itself have a small branching fraction; for points where this is not true our analysis must be modified. For $m_{\tilde{\chi}_{2}^{0}}>m_{\tilde{\chi}_{1}^{ \pm}}$the decay of the $\tilde{\chi}_{2}^{0}$ through a chargino, which then decays to an LSP, can be prominent, and can be a background to chargino events. The $\tilde{\chi}_{3}^{0}$ may also undergo cascade decays. To distinguish these cases, and to isolate the chargino signal, one should vary the beam energy and make use of the fact that each signal has a unique energy dependence. Our Monte Carlo simulation does not include these supersymmetric backgrounds, and we have not studied the effectiveness of changing the beam energy, but we will assume that an energy scan will allow the separation of the chargino signal from cascade decays. In any event, as the SUSY parameters become roughly known, it is possible that improved cuts could be devised to efficiently separate the chargino and neutralino signals.

Approximate knowledge of the SUSY parameters may also be relevant for the isolation of the chargino signal from standard model backgrounds. The most obvious example is the possibility of a chargino with mass less than $M_{W}$, in which case one could work below the $W W$ threshold, dispense with cuts 3-5, and increase the number of signal events by approximately a factor of 2 . However, for simplicity in this exploratory study, we will use cuts 1-4 and the requirement on quark energies in all regions of parameter space.

\section{CASE STUDIES}

In this section, we present a number of case studies at representative points in SUSY parameter space. In the first subsection, we discuss the general procedure that will be used to find the allowed regions of SUSY parameter space. We also describe the way in which our results will be presented graphically. In the remaining subsections, we consider points in the gaugino, Higgsino, and mixed regions, and determine for each case how well the observables may be measured and what bounds on underlying SUSY parameters may be obtained. Throughout this section, we present results for $\sqrt{s}=190 \mathrm{GeV}$ and an event sample of $1 \mathrm{fb}^{-1}$.

\section{A. Strategy for Finding Allowed Parameter Space}

After the observables $m_{\tilde{\chi}_{1}^{ \pm}}, m_{\tilde{\chi}_{1}^{0}}, \sigma_{\text {total }}$, and $B_{l}$ are measured, one must determine how the SUSY parameter space is restricted. As the parameter space is six-dimensional, it is important to outline a method by which such restrictions are easily applied and understood.

The dependence of $m_{\tilde{\chi}_{1}^{ \pm}}$on only three SUSY parameters allows us a simple starting point. First, consider the three-dimensional space $\left(\mu, M_{2}, \tan \beta\right)$. A point $\mathcal{P}$ in this space survives the chargino mass measurement if it predicts the chargino mass correctly, within experimental uncertainties. The allowed region is then confined to two thin sheets which we will label as $\mathcal{S}$, one with $\mu<0$ and another with $\mu>0$. This is shown schematically in Fig. 14. 
To display our results, it will be necessary to plot contours in the allowed region, and it will therefore be convenient to flatten the two sheets $\mathcal{S}$ into a plane $\mathcal{T}$ with the coordinate transformation

$$
\left(\mu, M_{2}, \tan \beta\right) \in \mathcal{S} \rightarrow\left(\alpha \equiv \arctan \frac{M_{2}}{\mu}, \tan \beta\right) \in \mathcal{T}
$$

as shown in Fig. 14. Since the sheets are not infinitely thin, a short segment of points in $\mathcal{S}$ is projected into every point in $\mathcal{T}$. The far gaugino regions are then transformed to the areas with $\alpha \approx 0^{\circ}, 180^{\circ}$, and the far Higgsino regions now correspond to the region with $\alpha \approx 90^{\circ}$. The symmetry $\mu \leftrightarrow-\mu$ for $\tan \beta \rightarrow \infty$ implies that, at large $\tan \beta$, observables at $\alpha$ are nearly equal to those at $180^{\circ}-\alpha$.

The allowed region is restricted further by the other measurements. The LSP mass $m_{\tilde{\chi}_{1}^{0}}$ is a function of $\mu, M_{2}, \tan \beta$, and $M_{1}$, and so the $m_{\tilde{\chi}_{1}^{0}}$ measurement limits $M_{1}$ to a certain range. To graphically represent this restriction of $M_{1}$, or equivalently, $M_{1} / M_{2}$, we will do the following. For a point $\mathcal{P}=(\alpha, \tan \beta) \in \mathcal{T}$, we find all parameters $\left(\mu, M_{2}=\right.$ $\left.\mu \tan \alpha, \tan \beta, M_{1} / M_{2}\right)$ such that the corresponding values of $m_{\tilde{\chi}_{1}^{ \pm}}$and $m_{\tilde{\chi}_{1}^{0}}$ are within the experimental limits. The allowed values of $M_{1} / M_{2}$ will lie in some range $\left(M_{1} / M_{2}\right)_{\min }<$ $M_{1} / M_{2}<\left(M_{1} / M_{2}\right)_{\max }$. To display this range, we will plot contours in $\mathcal{T}$ of $\left(M_{1} / M_{2}\right)_{\min }$ and $\left(M_{1} / M_{2}\right)_{\max }$. If no value for $M_{1}$ in the range given in Eq. (18) leads to the correct $m_{\tilde{\chi}_{1}^{0}}$, the point $\mathcal{P}$ is excluded.

In a similar manner, the measurement of $\sigma_{\text {total }}\left(\mu, M_{2}, \tan \beta, m_{\tilde{l}}\right)$ will limit the allowed range of $m_{\tilde{l}}$, and this range can be represented in contour plots of $\left(m_{\tilde{l}}\right)_{\min }$ and $\left(m_{\tilde{l}}\right)_{\max }$. If no value of $m_{\tilde{l}}$ gives the correct $\sigma_{\text {total }}, \mathcal{P}$ is not allowed. Finally, given $\mathcal{P}$ and the ranges of $M_{1}$ and $m_{\tilde{l}}$ as determined above, the measured value of $B_{l}$ restricts the range of $m_{\tilde{q}}$ to a certain range, and we will also present contours of $\left(m_{\tilde{q}}\right)_{\min }$ and $\left(m_{\tilde{q}}\right)_{\max }$. If no value of $m_{\tilde{q}}$

yields the appropriate $B_{l}$, then the point $\mathcal{P}$ is excluded. In principle, measurement of $A_{F B}^{j j}$ may also be used to limit the allowed region, but we defer discussion of this observable to the individual case studies below.

The remaining points $\mathcal{P}=(\alpha, \tan \beta) \in \mathcal{T}$, for which there exist parameters $\left(\mu, M_{2}=\right.$ $\left.\mu \tan \alpha, \tan \beta, M_{1}, m_{\tilde{l}}, m_{\tilde{q}}\right)$ that are consistent with all measurements, form the allowed region. We will plot this region in the $\mathcal{T}$ plane. From this plot, the allowed ranges of $\rho_{\tilde{\chi}_{1}^{ \pm}}$ and $\tan \beta$ may be quickly determined. The ranges of and correlations between the other variables may be found from the contour plots of their minimum and maximum allowed values.

\section{B. Gaugino Region}

The first case we consider is a set of SUSY parameters in the gaugino region with gaugino mass unification. We choose

$$
\left(\mu, M_{2}, \tan \beta, M_{1} / M_{2}, m_{\tilde{l}}, m_{\tilde{q}}\right)=(-400,75,4,0.5,200,300) .
$$

With these parameters, $\alpha=169^{\circ}$, and the underlying values and ranges of the most important quantities are 


$$
\begin{aligned}
m_{\tilde{\chi}_{1}^{ \pm}} & =79.6 \mathrm{GeV} \\
m_{\tilde{\chi}_{1}^{0}} & =39.1 \mathrm{GeV} \\
\sigma_{\text {total }} & =1.16 \mathrm{R}=3200 \mathrm{fb} \\
\rho_{\tilde{\chi}_{1}^{ \pm}} & =1.00 \\
\rho_{\tilde{\chi}_{1}^{0}} & =0.99 \\
B_{l} & =0.42 \\
m_{j j} & <40.5 \mathrm{GeV} \\
16.4 \mathrm{GeV} & <E_{j j}<55.8 \mathrm{GeV} .
\end{aligned}
$$

Since $m_{\tilde{\chi}_{1}^{ \pm}} \approx M_{W}$, it is not possible to work below the $W W$ threshold, and the cuts for $2 j+l$ events described in Sec. [V are likely to be nearly ideal. Note that the sneutrino mass has been taken near the low end of the range. This value leads to substantial destructive interference in the production amplitude; higher values for $m_{\tilde{\nu}}$ would give considerably larger cross sections.

With an integrated luminosity of $1 \mathrm{fb}^{-1}$, there are 3203 chargino events, and the Monte Carlo simulation yields 1493 mixed mode events. Some of these events include hadronicallydecaying $\tau$ leptons, but the rest of them are $Y$ mode events, as defined in Sec. II. In the Monte Carlo simulation we are left with $1184 Y$ events, of which 889 (75\%) survive the cuts described in Sec. IV. In addition to these $Y$ mode events, some leptonic mode chargino events with hadronically-decaying $\tau$ leptons will also pass the cuts. However, hadrons resulting from $\tau$ decays are highly collimated with invariant mass less than $m_{\tau}$. The $m_{j j}$ spectrum for the $889 Y$ events is shown in Fig. 15a. Clearly, very few $Y$ mode events have dijet masses consistent with $\tau$ decays, and $Y$ events should be easily separated on this basis. We will therefore assume that we have an event sample of $Y$ events that is virtually free of background and may be used to determine the values of chargino event observables.

We may now determine the masses $m_{\tilde{\chi}_{1}^{ \pm}}$and $m_{\tilde{\chi}_{1}^{0}}$ from the endpoints of dijet mass and energy distributions, as discussed in Sec. III. These distributions are given in Fig. 15. We see that finite detector resolution effects cause the spectra to have tails that exceed the theoretical limits, but despite this, the endpoints are fairly sharp. The $m_{j j}^{\max }$ endpoint almost certainly lies within a $8 \mathrm{GeV}$ range, and we therefore estimate its $1 \sigma$ error to be 2 $\mathrm{GeV}$. Similarly, we estimate that the $1 \sigma$ error for the maximum endpoint of $E_{j j}$ is $3 \mathrm{GeV}$. As noted in Sec. IV, these resolutions are expected to improve with the addition of tracking chamber momentum measurements 44,45], and we therefore take the actual resolutions to be reduced by $25 \%$ to $\Delta m_{j j}^{\max }=1.5 \mathrm{GeV}$ and $\Delta E_{j j}^{\max }=2.3 \mathrm{GeV}$. (In the next subsection we will examine the effect on our results of increasing these uncertainties.) We must now propagate these uncertainties into the determinations of the underlying masses. The relevant formulae for the uncertainty calculations are collected in the appendix. From Eqs. (A1) and (A2) we find

$$
\Delta m_{\tilde{\chi}_{1}^{ \pm}}=2.5 \mathrm{GeV} \text { and } \Delta m_{\tilde{\chi}_{1}^{0}}=2.2 \mathrm{GeV} .
$$

For simplicity, we have assumed that the central values for endpoint measurements are their underlying physical values. We note also that, although $E_{j j}^{\min }$ provides a useful cross check and may also improve the mass determinations, we will not use it here.

Next we must determine $\sigma_{\text {total }}$ and $B_{l}$ and the uncertainties in their measurements. To do this, as discussed in Sec. III, we must measure at least two of the three partial 
cross sections $\sigma_{\text {mixed }}, \sigma_{\text {hadronic }}$, and $\sigma_{\text {leptonic. }}$ The partial cross section $\sigma_{\text {mixed }}$ is always one of the two largest, and we will consider this mode in detail. As noted above, we do not measure $\sigma_{\text {mixed }}$ directly, but rather $\sigma_{Y}$. Under the assumptions of Sec. II], which imply lepton universality, $\sigma_{Y}=\frac{2.0+0.4}{3.0} \sigma_{\text {mixed }}=\frac{4}{5} \sigma_{\text {mixed }}$ and $\frac{\Delta \sigma_{Y}}{\sigma_{Y}}=\frac{\Delta \sigma_{\text {mixed }}}{\sigma_{\text {mixed }}}$. We will then assume that similar errors, in a sense to be defined precisely below, may be obtained for the hadronic mode. With these assumptions, we then find the uncertainties of $\sigma_{\text {total }}$ and $B_{l}$. (The consistency of our assumption of lepton universality may be checked by verifying that, for example, $B\left(\tilde{\chi}_{1}^{+} \tilde{\chi}_{1}^{-} \rightarrow 2 j+e\right)=B\left(\tilde{\chi}_{1}^{+} \tilde{\chi}_{1}^{-} \rightarrow 2 j+\mu\right)$. Furthermore, if additional branching ratios can be measured, one may determine directly whether lepton universality indeed holds.)

We must now determine the uncertainty for measurements of $\sigma_{Y}$. For any mode $i$, the cross section and fractional uncertainty are

$$
\sigma_{i}=N_{i} \eta_{i}^{-1} \mathcal{L}^{-1}
$$

and

$$
\frac{\Delta \sigma_{i}}{\sigma_{i}}=\left[\left(\frac{\Delta N_{i}}{N_{i}}\right)^{2}+\left(\frac{\Delta \eta_{i}}{\eta_{i}}\right)^{2}+\left(\frac{\Delta \mathcal{L}}{\mathcal{L}}\right)^{2}\right]^{\frac{1}{2}}
$$

where $N_{i}$ is the number of $i$ mode events passing the cuts, $\eta_{i}$ is the efficiency of the cuts for $i$ mode events, and $\mathcal{L}$ is the collider luminosity. The number of $Y$ mode events passing the cuts is 889 , so $\Delta N_{Y} / N_{Y}=1 / \sqrt{N_{Y}}=3.4 \%$. The efficiency $\eta_{Y}$ is not known and, in principle, depends on all the SUSY parameters that we are trying to determine. However, by running Monte Carlo simulations for many points in SUSY parameter space with the measured $m_{\tilde{\chi}_{1}^{ \pm}}$and $m_{\tilde{\chi}_{1}^{0}}$, we can determine how much the efficiency varies throughout the allowed parameter space. We have done this for points in the gaugino, Higgsino, and mixed regions, for both positive and negative $\mu$, various $\tan \beta$, and $\left(m_{\tilde{l}}, m_{\tilde{q}}\right)=(100,150),(200$, $300)$, and $(500,700)$, with the sole restriction being that these points give $m_{\tilde{\chi}_{1}^{ \pm}} \approx 80 \mathrm{GeV}$ and $m_{\tilde{\chi}_{1}^{0}} \approx 39 \mathrm{GeV}$. For all of these cases, the efficiency of the cuts varies only between $70 \%$ and $77 \%$. Thus, the cut efficiency is determined primarily by kinematics and varies only slightly for fixed $m_{\tilde{\chi}_{1}^{ \pm}}$and $m_{\tilde{\chi}_{1}^{0}}$. We take the efficiency to be $\eta_{Y}=73.5 \%$ and its variation to be $\Delta \eta_{Y}=3.5 \%$. The uncertainty in the luminosity, which at LEP I is $\Delta \mathcal{L} / \mathcal{L}=0.3 \%$, and which is not expected to increase substantially for LEP II [46], is much smaller than the other errors. Substituting these values into Eq. (66), we find that $\Delta \sigma_{Y} / \sigma_{Y}=5.8 \%$.

Although we do not have specific cuts for the hadronic and leptonic modes, we will assume that cuts with similar $\eta$ and $\Delta \eta$ may be devised for at least one of the other modes. Such an assumption is certainly not to be taken for granted as the other two modes have large backgrounds. In the four jet mode, it may be possible to reduce backgrounds substantially by demanding that no pairing of jets yields two dijet masses consistent with $M_{W}$ or $M_{Z}$. The leptonic mode is plagued by an irreducible background from $W$ pair production [7]. As can be seen from Eq. (66), the best cuts for the purposes of this study are those that balance uniformity (low $\frac{\Delta \eta_{i}}{\eta_{i}}$ ) with background suppression and efficiency (low $\frac{\Delta N_{i}}{N_{i}}$ ). It is clear that detailed studies of cuts for the $4 j$ and $2 l$ events are necessary for future work in this area. For this study, however, we will calculate the fractional uncertainty in $\sigma_{\text {hadronic }}$ assuming that cuts may be devised with values of $\eta$ and $\Delta \eta$ similar to those obtained in 
the mixed mode. It is important to note that significantly worse values of $\eta_{\text {hadronic }}$ need not change our main results dramatically. We will demonstrate this explicitly in the following subsection, where results are presented for lower values of $\eta_{\text {hadronic }}$. We will assume in our case studies that the errors for the leptonic mode are larger than those for the hadronic mode, and so we will use only the mixed and hadronic modes for our determinations of $\sigma_{\text {total }}$ and $B_{l}$. In a complete analysis the leptonic mode should be combined with the others to further constrain the determinations of $\sigma_{\text {total }}$ and $B_{l}$.

The Monte Carlo simulation yields 1095 hadronic mode events. The assumption $\eta_{\text {hadronic }}=\eta_{Y}=73.5 \%$ implies $N_{\text {hadronic }}=804$, and assuming also that $\Delta \eta_{\text {hadronic }}=3.5 \%$, we find that $\Delta \sigma_{\text {hadronic }} / \sigma_{\text {hadronic }}=5.9 \%$. We may now proceed to determine the uncertainties in $\sigma_{\text {total }}$ and $B_{l}$. Using the formulae in the appendix, we find

$$
\Delta \sigma_{\text {total }} / \sigma_{\text {total }}=5.0 \% \text { and } \quad \Delta B_{l} / B_{l}=4.8 \% .
$$

We have now determined the uncertainties with which the four observables may be measured and can apply the strategy outlined in Sec. $\nabla \mathrm{A}$ to determine the allowed region in SUSY parameter space. To recapitulate, the $m_{\tilde{\chi}_{1}^{ \pm}}$measurement restricts the $\left(\mu, M_{2}, \tan \beta\right)$ space to two thin sheets, one with $\mu<0$ and another with $\mu>0$. These sheets are then flattened into the $(\alpha, \tan \beta)$ plane, where $\alpha$ is the angle given by $\tan \alpha=M_{2} / \mu$. The far gaugino regions are at $\alpha \approx 0^{\circ}, 180^{\circ}$, while the far Higgsino region lies near $\alpha \approx 90^{\circ}$.

For any given point $\mathcal{P}$ in the $(\alpha, \tan \beta)$ plane, we determine values of $M_{1} / M_{2}$ that give the measured value of $m_{\tilde{\chi}_{1}^{0}}$ within $1 \sigma$ bounds. In general, there will be an allowed range for $M_{1}>0$ and another for $M_{1}<0$. These are distinct branches, as there are no symmetries connecting positive and negative $M_{1}$. For now, let us investigate the $M_{1}>0$ possibility only.

In Fig. 16 we plot constant contours of the minimum and maximum allowed values of $M_{1} / M_{2}$ in the $(\alpha, \tan \beta)$ plane. The approximate symmetry $\mu \leftrightarrow-\mu$ for large $\tan \beta$ is already in evidence at $\tan \beta=10$, and the contour lines are approximately independent of $\tan \beta$ above this value. If no value of $M_{1} / M_{2}$ gives the correct $m_{\tilde{\chi}_{1}^{0}}$, the point $(\alpha, \tan \beta)$ is excluded, as happens in the small cross-hatched area in the $\alpha>90^{\circ}(\mu<0)$ mixed region with $\tan \beta \approx 1$, where it is not possible to raise $M_{1} / M_{2}$ sufficiently to produce the required $m_{\tilde{\chi}_{1}^{0}}$. For the areas of the plane that are not excluded, the range of allowed $M_{1} / M_{2}$ values is quite restricted. In the far gaugino region, the allowed range of $M_{1} / M_{2}$ is roughly $0.45 \lesssim M_{1} / M_{2} \lesssim 0.55$ and is centered around 0.5 , as expected. In the Higgsino region, for decreasing $\rho_{\tilde{\chi}_{1}^{ \pm}}, M_{1} / M_{2}$ drops to zero. This is easily understood, since, as we approach the far Higgsino region, for which $M_{2}$ is large, $M_{1}$ must remain roughly constant at $M_{1} \sim 40 \mathrm{GeV}$ to accommodate the neutralino mass $m_{\tilde{\chi}_{1}^{0}} \approx 40 \mathrm{GeV}$. We also see that, for $\tan \beta \gtrsim 2.5$, only values of $M_{1} / M_{2}$ less than 0.6 are allowed.

We now determine the values of $m_{\tilde{l}}$ that give the observed $\sigma_{\text {total }}$ within $1 \sigma$ bounds. In Fig. 17 we plot the minimum and maximum allowed values of $m_{\tilde{l}}$ for points in the $(\alpha, \tan \beta)$ plane. In the far gaugino region, the slepton mass range is $180 \mathrm{GeV} \lesssim m_{\tilde{l}} \lesssim 220 \mathrm{GeV}$. The bounds are quite stringent because the cross section is very sensitive to $m_{\tilde{\nu}}$ in the gaugino region, as was shown in Fig. 10. As one moves from the gaugino region to the Higgsino region, the cross section for a fixed $m_{\tilde{l}}$ decreases, and therefore $m_{\tilde{l}}$ increases to compensate. In addition, the cross sections become less sensitive to $m_{\tilde{l}}$, and the uncertainty for the $m_{\tilde{l}}$ determination grows. Finally, at a certain point, $m_{\tilde{l}}$ cannot be large enough to prevent the 
cross section from dipping below the measured value, and thus the far Higgsino region is excluded. The $\sigma_{\text {total }}$ measurement alone is therefore enough to exclude the cross-hatched region of the $(\alpha, \tan \beta)$ plane in Fig. 17.

For the remaining allowed regions of the $(\alpha, \tan \beta)$ plane, we use the determined ranges of $M_{1}$ and $m_{\tilde{l}}$ to find the values of $m_{\tilde{q}}$ that give the correct $B_{l}$ within $1 \sigma$. Contours of constant $\left(m_{\tilde{q}}\right)_{\min }$ and $\left(m_{\tilde{q}}\right)_{\max }$ are plotted in Fig. 18, where the $\alpha>90^{\circ}(\mu<0)$ gaugino region has been enlarged. (Similar features are seen in the $\alpha<90^{\circ}(\mu>0)$ gaugino region.) As one approaches the far gaugino region, the leptonic branching fraction grows for fixed $m_{\tilde{q}}$, and the maximum allowed squark mass $\left(m_{\tilde{q}}\right)_{\max }$ decreases. At some point, no squark mass greater than $150 \mathrm{GeV}$ is allowed, and the hatched region is therefore excluded. Since $B_{l}$ grows more quickly for large $\tan \beta$, as predicted by Eqs. (54) and (56), the excluded region is larger for high $\tan \beta$. Conversely, if one moves from the far gaugino region to the gaugino region, $B_{l}$ drops, and to stay within the $1 \sigma$ bounds on $B_{l},\left(m_{\tilde{q}}\right)_{\min }$ grows. At a certain point, no $m_{\tilde{q}}$ is large enough to accommodate the measured $B_{l}$, and so the cross-hatched region is also excluded. The resulting allowed regions are shown in Fig. 19, where the cross-hatched regions are excluded. We see immediately that the allowed regions lie completely in the gaugino region. (A subtle point should be mentioned here. The $B_{l}$ measurement not only constrains the allowed region in the $(\alpha, \tan \beta)$ plane, but also, for a fixed point in the allowed region, further limits the acceptable values of $M_{1}$ and $m_{\tilde{l}}$. Therefore the allowed ranges may be somewhat reduced from those in Figs. 16 and 17. The effect is typically small, however, and so we do not present updated figures for $M_{1}$ and $m_{\tilde{l}}$ with the $B_{l}$ constraint imposed. Nonetheless, the full $B_{l}$ constraint is included in the results presented below.)

It is evident from the figures that the four measurements constrain the parameter space significantly, and the allowed ranges of the SUSY parameters are highly correlated. It is also useful to determine the global bounds that may be placed on the various quantities of interest, independent of their correlations. To determine the allowed ranges of these quantities, we pick points randomly in the allowed volume, and verify graphically that enough points have been picked to adequately sample the region. We find the following global bounds:

$$
\begin{aligned}
& 0.97<\rho_{\tilde{\chi}_{1}^{ \pm}}<1.00 \\
& 0.97<\rho_{\tilde{\chi}_{1}^{0}}<1.00 \\
& 180 \mathrm{GeV}<m_{\tilde{l}}<225 \mathrm{GeV} \\
& 0.43<\frac{M_{1}}{M_{2}}<0.58 \\
& -1 \mathrm{TeV}<\mu<-290 \mathrm{GeV} \quad \text { or } \quad 300 \mathrm{GeV}<\mu<1 \mathrm{TeV} \\
& 63 \mathrm{GeV}<M_{2}<93 \mathrm{GeV} \\
& 1<\tan \beta<50 \\
& 150 \mathrm{GeV}<m_{\tilde{q}}<1 \mathrm{TeV} \text {. }
\end{aligned}
$$

To understand the confidence level of these bounds, recall that the uncertainties in observables we have used are one standard deviation. The allowed region consists of all points in parameter space for which the central values of all observables are within $1 \sigma$ of the underlying physical values. Typically, the extremes of the quantities given in Eq. (68) are reached in corners of the allowed region for which more than one of the observables deviates by $1 \sigma$.

These bounds follow from the assumption that $M_{1}>0$. For the case of $M_{1}<0$, the resulting bounds are only very slightly weaker. For general $M_{1}$, we find that for the gaugino 
region point we have chosen, the global bounds are

$$
\begin{aligned}
& 0.97<\rho_{\tilde{\chi}_{1}^{ \pm}}<1.00 \\
& 0.97<\rho_{\tilde{\chi}_{1}^{0}}<1.00 \\
& 179 \mathrm{GeV}<m_{\tilde{l}}<227 \mathrm{GeV} \\
& -0.61<\frac{M_{1}}{M_{2}}<-0.45 \quad \text { or } \quad 0.43<\frac{M_{1}}{M_{2}}<.58 \\
& -1 \mathrm{TeV}<\mu<-290 \mathrm{GeV} \quad \text { or } \quad 300 \mathrm{GeV}<\mu<1 \mathrm{TeV} \\
& 63 \mathrm{GeV}<M_{2}<93 \mathrm{GeV} \\
& 1<\tan \beta<50, \\
& 150 \mathrm{GeV}<m_{\tilde{q}}<1 \mathrm{TeV} .
\end{aligned}
$$

The allowed regions in Fig. 19 are virtually unchanged when negative $M_{1}$ is included.

Even though correlations between variables are ignored, the global bounds of Eq. (69) have interesting implications. The gaugino content has been tightly constrained to be greater than 0.9, which supports the LSP as a dark matter candidate. For the case of $M_{1}>0$, the ratio $M_{1} / M_{2}$ has been determined to be compatible with grand unification to within approximately 15\%. There is, however, an allowed range of negative $M_{1}$. (In general, it is very difficult to exclude negative $M_{1}$ with the four observables we have explored.) The bound on $m_{\tilde{l}}$ is strong, as a result of the large destructive effect of the electron sneutrino on the total cross section. In many models the sneutrino is the next lightest observable SUSY particle, and this bound provides an important guide for future sparticle searches. Finally, $\tan \beta$ is unrestricted, and there is no global bound on $m_{\tilde{q}}$ - the squark mass may lie anywhere in the range we have considered. However, as seen in Fig. 18, at a given point in the $(\alpha, \tan \beta)$ plane, the bounds on $m_{\tilde{q}}$ may be quite strong.

We now turn to $A_{F B}^{\tilde{\chi}^{ \pm}}$and $A_{F B}^{j j}$, the forward-backward asymmetries discussed in Sec. III. We remind the reader that the former, while unobservable, depends only on the production amplitude, while the latter is observable but depends on the decay vertices as well. In Fig. 20, we plot $A_{F B}^{j j}$ vs. $A_{F B}^{\tilde{\chi}^{ \pm}}$for a number of points in the allowed region. The value of $A_{F B}^{j j}$ for our case study, measured from the $Y$ mode events that pass the cuts, is given by the solid line. Its $1 \sigma$ deviation, as determined from Eq. (A8), is given by the dashed lines.

Because the previous four observables have already restricted the allowed region to a small volume in the gaugino region with a light sneutrino, $A_{F B}^{\tilde{\chi}^{ \pm}}$is limited to the fairly narrow range $0.12 \lesssim A_{F B}^{\tilde{\chi}^{ \pm}} \lesssim 0.21$. As discussed in Sec. III, $A_{F B}^{\tilde{\chi}^{ \pm}}$and $\sigma_{\text {total }}$ are the only production quantities with much resolving power, so the fact that $A_{F B}^{\tilde{\chi}^{ \pm}}$is limited to a small range is evidence that we have already obtained nearly all of the information contained in the production amplitude.

We also see that the correlation between $A_{F B}^{j j}$ and $A_{F B}^{\tilde{\chi}^{ \pm}}$is weak, and that $A_{F B}^{j j}$ lies in a much broader range, $-0.06 \lesssim A_{F B}^{j j} \lesssim 0.3$. Clearly the decay amplitude plays a crucial role in determining the value of $A_{F B}^{j j}$, and the large variation in $A_{F B}^{j j}$ is an indication that detailed studies of chargino decays may improve the bounds on parameter space and tighten the correlations between variables. By running Monte Carlo simulations for a large number of points that densely populate the allowed region, one could presumably form a detailed picture of the regions that may be excluded on the basis of $A_{F B}^{j j}$. However, as our goal in this study is to study chargino events analytically, we will not discuss this possibility further. Still, even from our sparse sampling of the allowed region it is possible to draw some tentative 
conclusions. For example, in Fig. 20, points with positive $\mu$ have been marked with open circles, and those with negative $\mu$ have been marked with filled circles. We see that all of the points with $\mu>0$ may be excluded based on the $A_{F B}^{j j}$ measurement, which suggests that the $\mu>0$ portion of the allowed region may be eliminated by the $A_{F B}^{j j}$ measurement.

\section{Variations in $\frac{M_{1}}{M_{2}}$ and Experimental Assumptions}

We will now briefly explore two simple variations on the previous case study. In that example, we assumed gaugino mass unification and saw that it could be verified to $15 \%$ (assuming $M_{1}>0$ ). In our first variation below, we take $M_{1} / M_{2}=0.7$ and find how strongly the gaugino mass unification condition may be disfavored. In the second variation, we determine the impact of more pessimistic assumptions about detector resolutions and backgrounds. As these are only slight variations on the previous example, few new features appear in the analysis, and we will only present a few intermediate results and the final conclusions.

We begin by taking the parameters

$$
\left(\mu, M_{2}, \tan \beta, M_{1} / M_{2}, m_{\tilde{l}}, m_{\tilde{q}}\right)=(-400,75,4,0.7,200,300),
$$

where the only change from the previous example is that we choose $M_{1} / M_{2}=0.7$. Many of the basic quantities remain the same, but we list them all for convenience:

$$
\begin{aligned}
m_{\tilde{\chi}_{1}^{ \pm}} & =79.6 \mathrm{GeV} \\
m_{\tilde{\chi}_{1}^{0}} & =53.8 \mathrm{GeV} \\
\sigma_{\text {total }} & =1.16 \mathrm{R}=3202 \mathrm{fb} \\
\rho_{\tilde{\chi}_{1}^{ \pm}} & =1.00 \\
\rho_{\tilde{\chi}_{1}^{0}} & =0.99 \\
B_{l} & =0.40 \\
m_{j j} & <25.7 \mathrm{GeV} \\
11.7 \mathrm{GeV} & <E_{j j}<39.9 \mathrm{GeV} .
\end{aligned}
$$

Of the 3203 chargino events, the Monte Carlo simulation yields 1528 mixed mode events. 1210 of these are $Y$ mode events, and 715 (59\%) of these survive the cuts. Because the LSP is heavier, the ranges of the dijet mass and energy are smaller than in the original example. From plots of the dijet mass and energy distributions similar to those previously presented, we estimate that both $m_{j j}^{\max }$ and $E_{j j}^{\max }$ may be determined to $2 \mathrm{GeV}$. Including the $25 \%$ reduction in these uncertainties from the tracking chamber measurements, we find that the chargino and LSP masses are determined to

$$
\Delta m_{\tilde{\chi}_{1}^{ \pm}}=2.9 \mathrm{GeV} \quad \text { and } \quad \Delta m_{\tilde{\chi}_{1}^{0}}=2.0 \mathrm{GeV} \text {. }
$$

The efficiency $\eta_{Y}$ is found, as in the previous example, by running Monte Carlo simulations for a large number of points in SUSY parameter space with the correct $m_{\tilde{\chi}_{1}^{ \pm}}$and $m_{\tilde{\chi}_{1}^{0}}$. We find once again that the efficiency is principally determined by kinematics and lies in the range $54-62 \%$. We take $\eta_{Y}=58 \%$ and $\Delta \eta_{Y}=4 \%$, and assume similar values for the hadronic mode. Applying Eqs. (66) and (A4), we find the uncertainties 


$$
\Delta \sigma_{\text {total }} / \sigma_{\text {total }}=6.6 \% \text { and } \Delta B_{l} / B_{l}=6.4 \% .
$$

We now apply these measurements to the SUSY parameter space. By randomly sampling SUSY parameter space with both signs of $M_{1}$, we have found the following bounds in the allowed region:

$$
\begin{aligned}
& 0.78<\rho_{\tilde{\chi}_{1}^{ \pm}}<1.00 \\
& 0.84<\rho_{\tilde{\chi}_{1}^{0}}<1.00 \\
& 175 \mathrm{GeV}<m_{\tilde{l}}<233 \mathrm{GeV} \\
& -1.00<\frac{M_{1}}{M_{2}}<-0.38 \quad \text { or } \quad 0.60<\frac{M_{1}}{M_{2}}<1.00 \\
& -1 \mathrm{TeV}<\mu<-188 \mathrm{GeV} \quad \text { or } \quad 230 \mathrm{GeV}<\mu<1 \mathrm{TeV} \\
& 54 \mathrm{GeV}<M_{2}<119 \mathrm{GeV} \\
& 1<\tan \beta<50 \text {, } \\
& 150 \mathrm{GeV}<m_{\tilde{q}}<1 \mathrm{TeV} .
\end{aligned}
$$

The bounds on $M_{1} / M_{2}$ disfavor the gaugino mass unification hypothesis. The other conclusions and bounds are slightly weakened relative to the original gaugino case study, but we still obtain strong bounds on $m_{\tilde{l}}$ and find that the allowed region lies primarily in the gaugino region.

We now study the effects of varying our experimental assumptions. In the analysis above we have attempted to estimate the effects of finite detector resolutions and of backgrounds in the hadronic mode. Detailed studies and simulations are needed to significantly improve the accuracy of these estimates. We will show here, however, that most of the global bounds presented in the previous section are robust and are not altered greatly by assuming poorer experimental conditions. First, we modify the case study of Sec. VB by assuming that the backgrounds to the hadronic mode are very large, and that the optimal cuts have an efficiency $\eta_{\text {hadronic }}=18 \%$, which is $1 / 4$ of the value we took previously. We retain the estimate $\Delta \eta=3.5 \%$. The uncertainties in $m_{\tilde{\chi}_{1}^{ \pm}}$and $m_{\tilde{\chi}_{1}^{0}}$ remain the same, but we now find

$$
\begin{aligned}
\Delta \sigma_{\text {hadronic }} / \sigma_{\text {hadronic }} & =20.3 \% \\
\Delta \sigma_{\text {total }} / \sigma_{\text {total }} & =5.9 \% \\
\Delta B_{l} / B_{l} & =12.2 \% .
\end{aligned}
$$

Because we have assumed a low $\eta_{\text {hadronic }}$, the uncertainties in $\sigma_{\text {hadronic }}$ and $B_{l}$ are large. However, the uncertainty in $\sigma_{\text {total }}$ is not strongly affected because $1-2 B_{l}$ is small for this case study (see Eq. (A4)).

The analysis is identical to the previous example, so we skip the intermediate steps and present the end result. In the allowed region with both positive and negative $M_{1}$ values, the bounds on selected quantities are:

$$
\begin{aligned}
0.88 & <\rho_{\tilde{\chi}_{1}^{ \pm}}<1.00 \\
0.94 & <\rho_{\tilde{\chi}_{1}^{0}}<1.00 \\
176 \mathrm{GeV} & <m_{\tilde{I}}<231 \mathrm{GeV} \\
-1.00<\frac{M_{1}}{M_{2}}<-0.30 & \text { or } 0.42<\frac{M_{1}}{M_{2}}<0.80 .
\end{aligned}
$$

We see that the bounds are for the most part only slightly weakened, with the exception that the upper bound on positive $M_{1} / M_{2}$ is now 0.8 , and the lower bound on negative $M_{1} / M_{2}$ is -1.0 . 
We have also investigated the implications of doubling the estimated uncertainties on the dijet mass and energy endpoint determinations. In this variation, we take $\Delta m_{j j}^{\max }=4.0 \mathrm{GeV}$ and $\Delta E_{j j}^{\max }=6.0 \mathrm{GeV}$. In addition, we assume no improvement from tracking chamber measurements, and retain the "poor" hadronic mode efficiency of $\eta_{\text {hadronic }}=18 \%$. The uncertainties in $\sigma_{\text {total }}$ and $B_{l}$ are as in Eq. (75), and the new uncertainties in $m_{\tilde{\chi}_{1}^{ \pm}}$and $m_{\tilde{\chi}_{1}^{0}}$ are

$$
\Delta m_{\tilde{\chi}_{1}^{ \pm}}=6.7 \mathrm{GeV} \text { and } \quad \Delta m_{\tilde{\chi}_{1}^{0}}=5.8 \mathrm{GeV} .
$$

The resulting bounds are

$$
\begin{aligned}
0.83 & <\rho_{\tilde{\chi}_{1}^{ \pm}}<1.00 \\
0.91 & <\rho_{\tilde{\chi}_{1}^{0}}<1.00 \\
162 \mathrm{GeV} & <m_{\tilde{l}}<274 \mathrm{GeV} \\
-1.00<\frac{M_{1}}{M_{2}}<-0.26 & \text { or } 0.37<\frac{M_{1}}{M_{2}}<1.00 .
\end{aligned}
$$

In this case, we find that the bounds $M_{1} / M_{2}$ are weak. However, we find that we are still able to constrain the parameter space to the gaugino region and can place an upper bound on the sneutrino mass of $274 \mathrm{GeV}$. Thus, at least in the gaugino region, where our global bounds are expected to be the strongest, the limits on $\rho$ and $m_{\tilde{l}}$ are robust under variations in efficiency for the hadronic mode and in detector resolution.

\section{Higgsino Region}

For the Higgsino region case study, we choose the parameters

$$
\left(\mu, M_{2}, \tan \beta, M_{1} / M_{2}, m_{\tilde{l}}, m_{\tilde{q}}\right)=(-75,250,4,0.5,200,300) .
$$

For this point, the angle $\alpha=107^{\circ}$, and

$$
\begin{aligned}
m_{\tilde{\chi}_{1}^{ \pm}} & =79.7 \mathrm{GeV} \\
m_{\tilde{\chi}_{1}^{0}} & =62.3 \mathrm{GeV} \\
\sigma_{\text {total }} & =0.89 \mathrm{R}=2450 \mathrm{fb} \\
\rho_{\tilde{\chi}_{1}^{ \pm}} & =0.17 \\
\rho_{\tilde{\chi}_{1}^{0}} & =0.087 \\
B_{l} & =0.34 \\
m_{j j} & <17.4 \mathrm{GeV} \\
8.4 \mathrm{GeV} & <E_{j j}<28.6 \mathrm{GeV} .
\end{aligned}
$$

This point in parameter space is again fairly central in the accessible band, with $m_{\tilde{\chi}_{1}^{ \pm}} \approx$ $M_{W}$. It is possible to study points in parameter space that are closer to the pure Higgsino limit $\rho_{\tilde{\chi}_{1}^{ \pm}}=0$. However, as discussed in Sec. III, as one increases $\rho_{\tilde{\chi}_{1}^{ \pm}}$and $M_{2}, \tilde{\chi}_{1}^{ \pm}$and $\tilde{\chi}_{1}^{0}$ become more nearly degenerate, and the number of events with soft jets increases. These events are eliminated by our requirement that jet energies be greater than $5 \mathrm{GeV}$, and the resulting event sample is small. The point in parameter space given in Eq. (79) has been chosen to have properties characteristic of the Higgsino region, without being so far in the Higgsino region that low statistics become the primary concern. 
Given a sample of $1 \mathrm{fb}^{-1}$, there are 2454 events of which 1119 are mixed mode events. Considering only $Y$ mode events, that is, excluding the mixed events in which a $\tau$ decays hadronically, we are left with 893 events, of which 287 (32\%) survive the cuts described in Sec. IV. The efficiency of the cuts is lower than in the gaugino example because the smaller chargino-LSP mass splitting leads to more events with soft jets. In addition, since the LSPs are produced with lower velocities in the chargino rest frames and are more back-to-back in the lab frame, more events are eliminated by the $\not p_{T}$ cut.

We must now determine the masses from the endpoints of the $m_{j j}$ and $E_{j j}$ spectra, which are shown in Fig. 21. As in the gaugino case, finite detector resolution effects smear the endpoints, but we estimate that the maximum $m_{j j}$ endpoint almost certainly lies within a 4 $\mathrm{GeV}$ range, and therefore we takes its $1 \sigma$ error to be $1 \mathrm{GeV}$. Similarly, we estimate that the $1 \sigma$ error for the maximum endpoint of $E_{j j}$ is $2 \mathrm{GeV}$. As noted in Sec. IV, these resolutions are expected to improve with the addition of tracking chamber momentum measurements, and we therefore take the actual resolutions to be reduced by $25 \%$ to $\Delta m_{j j}^{\max }=0.75 \mathrm{GeV}$ and $\Delta E_{j j}^{\max }=1.5 \mathrm{GeV}$. (Again, we will not consider the lower $E_{j j}$ endpoint in the analysis here, although we expect it to be useful at least as a cross check.) The resulting uncertainties from Eqs. (A1) and (A2) for $m_{\tilde{\chi}_{1}^{ \pm}}$and $m_{\tilde{\chi}_{1}^{0}}$ are

$$
\Delta m_{\tilde{\chi}_{1}^{ \pm}}=3.0 \mathrm{GeV} \text { and } \Delta m_{\tilde{\chi}_{1}^{0}}=2.6 \mathrm{GeV} .
$$

We now turn to the determination of $\sigma_{\text {total }}$ and $B_{l}$. As in the gaugino region, the accuracy of these determinations depends on the statistical uncertainties and the variation of the cut efficiencies in the subvolume of SUSY parameter space with the given $m_{\tilde{\chi}_{1}^{ \pm}}$and $m_{\tilde{\chi}_{1}^{0}}$. With 287 events, the statistical uncertainty is $\Delta N_{Y} / N_{Y}=5.9 \%$. To determine the efficiency of the cuts, we have run Monte Carlo simulations for a wide range of representative points with $m_{\tilde{\chi}_{1}^{ \pm}} \approx 80 \mathrm{GeV}$ and $m_{\tilde{\chi}_{1}^{0}} \approx 62 \mathrm{GeV}$, and find that the cut efficiencies are fairly uniform and in the range of $26-34 \%$. We take the efficiency to be $\eta_{Y}=30 \%$ and its variation to be $\Delta \eta_{Y}=4 \%$. Combining these uncertainties as in the gaugino case, we find that $\Delta \sigma_{Y} / \sigma_{Y}=15 \%$. Finally, we will assume that the formulae in Eq. (A4) using the hadronic cross section are the ones with the smallest uncertainties. There are 1057 hadronic mode events in the Monte Carlo simulation. Again taking the assumption that $\eta_{\text {hadronic }} \approx \eta_{Y}$ and $\Delta \eta_{\text {hadronic }} \approx \Delta \eta_{Y}$, we find $\Delta \sigma_{\text {hadronic }} / \sigma_{\text {hadronic }}=14 \%$, and, from Eq. (A4),

$$
\Delta \sigma_{\text {total }} / \sigma_{\text {total }}=11 \% \text { and } \Delta B_{l} / B_{l}=14 \% \text {. }
$$

We may now use these four measurements to find the allowed parameter space. As in the gaugino example, $m_{\tilde{\chi}_{1}^{ \pm}}$limits us to two thin sheets in $\left(\mu, M_{2}, \tan \beta\right)$ space, and these are flattened into the plane $(\alpha, \tan \beta)$. We then determine the allowed region as in the gaugino case by applying the bounds on $m_{\tilde{\chi}_{1}^{0}}, \sigma_{\text {total }}$, and $B_{l}$ to determine ranges of $M_{1}, m_{\tilde{l}}$ and $m_{\tilde{q}}$ for every point in the plane. We will proceed as in the gaugino region case, first considering only $M_{1}>0$, and then including the possibility $M_{1}<0$ in the final determination of the allowed region.

The allowed range of $M_{1} / M_{2}$ from the $m_{\tilde{\chi}_{1}^{0}}$ measurement is shown in Fig. 22. As in Fig. 16 of the gaugino example, a portion of the low $\tan \beta, \mu<0$, mixed region is excluded, and the allowed ratio drops to zero in the far Higgsino region. Relative to the gaugino example, however, the central value of the allowed range of $M_{1} / M_{2}$ is increased in the 
gaugino region because now $m_{\tilde{\chi}_{1}^{1}} / m_{\tilde{\chi}_{1}^{ \pm}}=0.78$. The gaugino regions, in which $\rho_{\tilde{\chi}_{1}^{ \pm}}, \rho_{\tilde{\chi}_{1}^{0}}>0.9$, can be determined to lie within the regions $\alpha>135^{\circ}$ and $\alpha<30^{\circ}$. From Fig. 22, we see that $M_{1} / M_{2} \gtrsim 0.55$ in the gaugino region, and therefore, even including experimental uncertainties in the mass determinations, it is possible from measurements of $m_{\tilde{\chi}_{1}^{ \pm}}$and $m_{\tilde{\chi}_{1}^{0}}$ to determine that either the LSP is not a good dark matter candidate or the grand unification prediction of $M_{1} / M_{2}=0.5$ is not satisfied.

The $m_{\tilde{l}}$ bounds from $\sigma_{\text {total }}$ are shown in Fig. 23. The bounds in the gaugino region are again strong, as $\sigma_{\text {total }}$ is sensitive to $m_{\tilde{l}}$ in that region. We see that if the underlying parameters lie in the gaugino region, the bound $m_{\tilde{l}} \lesssim 250 \mathrm{GeV}$ applies, a promising result for scalar particle searches. The limits near $\alpha=90^{\circ}$ are not as strong, which is hardly surprising, since in the Higgsino region $\sigma_{\text {total }}$ is highly insensitive to $m_{\tilde{l}}$.

The bounds on $m_{\tilde{q}}$ from $B_{l}$ are presented in Fig. 24, where we have magnified two regions of parameter space that may be excluded based on the $B_{l}$ measurement. As we saw in Sec. [IIF, there is generically a pocket of small $B_{l}$ in the mixed $\mu<0$ region for moderate $\tan \beta$. In the cross-hatched, crescent-shaped excluded region in Fig. 24a, $B_{l}$ would be much smaller than the observed measurement of 0.34 even for the largest values of $m_{\tilde{q}}$. In Fig. 24b, we see that the hatched far gaugino region is excluded because $B_{l}$ would be too high, even for the lowest allowed value of $m_{\tilde{q}}$. For $\tan \beta=1$, this excluded region is for $\alpha \gtrsim 177^{\circ}$ $(\mu \lesssim-1.5 \mathrm{TeV})$, a region that is already disfavored by fine-tuning considerations. However, for larger $\tan \beta$, as discussed in Sec. ПIIF, $B_{l}$ grows more quickly as one approaches the far gaugino limit. The excluded region is therefore larger for higher $\tan \beta$, and, for $\tan \beta=10$, points with $\alpha \gtrsim 170^{\circ}(\mu \lesssim-450 \mathrm{GeV})$ are excluded.

Compiling these results, along with those for $M_{1}<0$, we find that the allowed regions are as given in Fig. 25. Although we have seen that a number of interesting correlations hold, it is clear that the global bounds will not be as impressive as in the gaugino case. Nevertheless, we present them here for completeness:

$$
\begin{aligned}
0.00 & <\rho_{\tilde{\chi}_{1}^{ \pm}}<1.00 \\
0.01 & <\rho_{\tilde{\chi}_{1}^{0}}<1.00 \\
100 \mathrm{GeV} & <m_{\tilde{l}}<1 \mathrm{TeV} \\
-1.00<\frac{M_{1}}{M_{2}}<-0.03 & \text { or } 0.10<\frac{M_{1}}{M_{2}}<0.99 \\
-870 \mathrm{GeV}<\mu<-65 \mathrm{GeV} & \text { or } \quad 79 \mathrm{GeV}<\mu<1 \mathrm{TeV} \\
62 \mathrm{GeV} & <M_{2}<1 \mathrm{TeV} \\
1 & <\tan \beta<50 \\
150 \mathrm{GeV} & <m_{\tilde{q}}<1 \mathrm{TeV} .
\end{aligned}
$$

In Fig. 26 we plot $A_{F B}^{j j}$ vs. $A_{F B}^{\tilde{\chi}^{ \pm}}$for a number of points in the allowed region. The solid line is the measured value of $A_{F B}^{j j}$, and the dashed lines are the $1 \sigma$ bounds. Although the points only sparsely sample the allowed region, it is evident that the relation between the production quantity $A_{F B}^{\tilde{\chi}^{ \pm}}$and the observed $A_{F B}^{j j}$ is heavily dependent on the decay process, and in fact, for the measured $A_{F B}^{j j}$, the full range of $A_{F B}^{\tilde{\chi}^{ \pm}}$values is possible. Without densely sampling the allowed region, it is difficult to reach any clear conclusions about the specific shape of the regions excluded by $A_{F B}^{j j}$, but it is likely that properties of the decay process will be useful in further reducing the allowed parameter space. 


\section{E. Mixed Region}

Finally, we turn to an example in the mixed region with parameters

$$
\left(\mu, M_{2}, \tan \beta, M_{1} / M_{2}, m_{\tilde{l}}, m_{\tilde{q}}\right)=(-90,115,4,0.5,200,300)
$$

for which $\alpha=128^{\circ}$ and

$$
\begin{aligned}
m_{\tilde{\chi}_{1}^{ \pm}} & =80.3 \mathrm{GeV} \\
m_{\tilde{\chi}_{1}^{0}} & =52.8 \mathrm{GeV} \\
\sigma_{\text {total }} & =0.75 \mathrm{R}=2070 \mathrm{fb} \\
\rho_{\tilde{\chi}_{1}^{ \pm}} & =0.64 \\
\rho_{\tilde{\chi}_{1}^{0}} & =0.60 \\
B_{l} & =0.32 \\
m_{j j} & <27.6 \mathrm{GeV} \\
12.6 \mathrm{GeV} & <E_{j j}<41.4 \mathrm{GeV} .
\end{aligned}
$$

This point in parameter space has been chosen to give the same $m_{\tilde{\chi}_{1}^{ \pm}}$as in the previous cases, and a value of $\rho$ that is between those of the earlier examples. For this point, the mass spectrum of the charginos and neutralinos has two features not present in the previous two cases. The second neutralino has mass $m_{\tilde{\chi}_{2}^{0}}=76.6 \mathrm{GeV}$, and is therefore lighter than the lighter chargino. This means that cascade decays of the chargino are kinematically possible. However, as $m_{\tilde{\chi}_{1}^{ \pm}}-m_{\tilde{\chi}_{2}^{0}} \gg m_{\tilde{\chi}_{1}^{ \pm}}-m_{\tilde{\chi}_{1}^{0}}$, and direct decays are not suppressed by any small couplings in the mixed region, cascade decays are highly suppressed relative to direct decays to the LSP, and we do not expect them to alter our analysis. The second new feature is that, since neither $M_{2}$ nor $\mu$ is large, even $\tilde{\chi}_{3}^{0}$ is light with mass $119 \mathrm{GeV}$. Thus, in this case not only are $\tilde{\chi}_{1}^{0} \tilde{\chi}_{2}^{0}$ and $\tilde{\chi}_{2}^{0} \tilde{\chi}_{2}^{0}$ production possible, but even $\tilde{\chi}_{1}^{0} \tilde{\chi}_{3}^{0}$ production is possible. The simultaneous production of all these signals may make chargino production difficult to resolve. However, we may remove part of this background by reducing the beam energy below the $\tilde{\chi}_{1}^{0} \tilde{\chi}_{3}^{0}$ production threshold. In this study we will assume that $\tilde{\chi}_{3}^{0}$ production can be separated from chargino production through this procedure, and we will ignore the effects of $\tilde{\chi}_{3}^{0}$ production as a background to chargino events. Despite possible difficulties from an entanglement of many supersymmetric signals, it should be kept in mind that every signal brings a wealth of new information, and generically the mixed region is the most, not the least, optimistic scenario. Though we will consider only the constraints that may be extracted from the chargino signal, the neutralino signals will lead to additional restrictions that should be imposed on the parameter space, and the full set of constraints from LEP II will most likely be stronger than our results would suggest.

In arriving at bounds for the more interesting quantities, we will skip many details as the method is identical to that employed in the previous cases. Given a sample of $1 \mathrm{fb}^{-1}$, there are 2072 events of which 907 are mixed mode events. Of these, 741 are $Y$ mode events, and $444(60 \%)$ of these survive the cuts of Sec. IV.

From plots of the distributions of $m_{j j}$ and $E_{j j}$, we estimate the endpoint uncertainties to be $3 \mathrm{GeV}$ for $m_{j j}^{\max }$ and $2 \mathrm{GeV}$ for $E_{j j}^{\max }$. Again assuming that tracking chamber measurements reduce these uncertainties by $25 \%$, we find that $\Delta m_{j j}^{\max }=1.5 \mathrm{GeV}$ and $\Delta E_{j j}^{\max }=2.3 \mathrm{GeV}$. The resulting uncertainties for $m_{\tilde{\chi}_{1}^{ \pm}}$and $m_{\tilde{\chi}_{1}^{0}}$ are 


$$
\Delta m_{\tilde{\chi}_{1}^{ \pm}}=3.3 \mathrm{GeV} \quad \text { and } \quad \Delta m_{\tilde{\chi}_{1}^{0}}=2.7 \mathrm{GeV} .
$$

To determine the uncertainties in the determinations of $\sigma_{\text {total }}$ and $B_{l}$, we must first determine $\Delta \sigma_{Y} / \sigma_{Y}$ from Eq. (65). With $444 Y$ mode events, the statistical uncertainty is $\Delta N_{Y} / N_{Y}=4.7 \%$. The efficiencies of the cuts for a wide range of representative points in SUSY parameter space, subject only to the restriction $m_{\tilde{\chi}_{1}^{ \pm}} \approx 80 \mathrm{GeV}$ and $m_{\tilde{\chi}_{1}^{0}} \approx 53 \mathrm{GeV}$, range from $54-62 \%$, and we therefore take the efficiency to be $\eta_{Y}=58 \%$ and its variation to be $\Delta \eta_{Y}=4 \%$. Combining these uncertainties, we find that $\Delta \sigma_{Y} / \sigma_{Y}=8.4 \%$. There are 975 Monte Carlo hadronic mode events before cuts, and again taking $\eta$ and $\Delta \eta$ to be approximately equal for the $Y$ and hadronic modes, we find $\Delta \sigma_{\text {hadronic }} / \sigma_{\text {hadronic }}=8.1 \%$. Using Eq. (A4) we determine that

$$
\Delta \sigma_{\text {total }} / \sigma_{\text {total }}=6.1 \% \text { and } \quad \Delta B_{l} / B_{l}=7.9 \% .
$$

Given these ranges for the four observables, we may now bound the parameter space. The allowed range of $M_{1} / M_{2}$ is shown in Fig. 27. In the gaugino region, the minimum value of $M_{1} / M_{2}$ is roughly 0.5 ; though values of $M_{1} / M_{2}>0.5$ are favored, the prediction of grand unified theories cannot be excluded. As in the previous two examples, a small $\mu<0$, $\tan \beta \approx 1$ region is ruled out.

The $m_{\tilde{l}}$ bounds from $\sigma_{\text {total }}$ are shown in Fig. 28. For this example $\sigma_{\text {total }}$ lies below the value of $\sigma_{\text {total }}$ approached in the pure Higgsino limit, as may be seen from Eq. (36) or Fig. 10. Thus, not only is some of the Higgsino region excluded, but also we obtain, from the $\sigma_{\text {total }}$ measurement alone, an upper bound on $m_{\tilde{l}}$. For $\tan \beta>4$ we see that the low value of $\sigma_{\text {total }}$ gives the bound $m_{\tilde{l}}<250 \mathrm{GeV}$.

Because $B_{l}$ is approximately $\frac{1}{3}$ as it was in the Higgsino example, the bounds on $m_{\tilde{q}}$ are fairly similar to those obtained in Fig. 24, so we will not present the $\left(m_{\tilde{q}}\right)_{\min }$ and $\left(m_{\tilde{q}}\right)_{\max }$ contours for this case study. It is again possible to rule out a crescent-shaped region in which $B_{l}$ is too small, and the far gaugino region in which $B_{l}$ is too large. The allowed region for the mixed region case study, considering both negative and positive $M_{1}$, is given in Fig. 29 . By randomly sampling the allowed region, we find the following global bounds:

$$
\begin{aligned}
& 0.05<\rho_{\tilde{\chi}_{1}^{ \pm}}<1.00 \\
& 0.01<\rho_{\tilde{\chi}_{1}^{0}}<1.00 \\
& 100 \mathrm{GeV}<m_{\tilde{l}}<257 \mathrm{GeV} \\
& -1.00<\frac{M_{1}}{M_{2}}<-0.12 \quad \text { or } \quad 0.16<\frac{M_{1}}{M_{2}}<1.00 \\
& -339 \mathrm{GeV}<\mu<-52 \mathrm{GeV} \quad \text { or } \quad 85 \mathrm{GeV}<\mu<355 \mathrm{GeV} \\
& 51 \mathrm{GeV}<M_{2}<500 \mathrm{GeV} \\
& 1<\tan \beta<50 \\
& 150 \mathrm{GeV}<m_{\tilde{q}}<1 \mathrm{TeV} \text {. }
\end{aligned}
$$

Again, the correlations among the various parameters are not represented in these limits. As already noted from Fig. 28, we see that the sneutrino mass bound is very stringent, with a maximum value of $257 \mathrm{GeV}$, as in the gaugino case. The other global bounds are weak.

Finally, we plot $A_{F B}^{j j}$ vs. $A_{F B}^{\tilde{\chi}^{ \pm}}$for a few points in the allowed region in Fig. 30. As in the previous figures, the solid line is the measured value of $A_{F B}^{j j}$, and the dashed lines are the $1 \sigma$ bounds. Although definite conclusions would require a more thorough sampling of 
the allowed region, Fig. 30 suggests that the point we have picked has an extreme value of $A_{F B}^{j j}$, and could therefore be distinguished from most other points in the allowed region by decay process considerations.

\section{FINAL COMMENTS AND SUMMARY}

We have explored the potential for precise determinations of fundamental SUSY parameters from chargino production at LEP II. We have found that chargino events can be well-described by six underlying SUSY parameters: $\mu, M_{2}, \tan \beta, M_{1}, m_{\tilde{l}}$, and $m_{\tilde{q}}$. A number of observables were investigated, and four - the chargino mass, the LSP mass, the total cross section, and the leptonic branching fraction - were found to be particularly useful in most areas of parameter space. These four observables, with their accompanying uncertainties, were used to restrict the allowed SUSY parameter space for representative points in the gaugino, Higgsino, and mixed regions, and a simple method for representing the results graphically was used.

In the gaugino region, we found stringent global bounds on SUSY parameters. In particular, $\rho_{\tilde{\chi}_{1}^{0}}$ was restricted to ranges in which the LSP is a good dark matter candidate, the gaugino mass unification condition could be verified or disproved at the level of $15 \%$, and an upper limit for the sneutrino mass could be obtained for an underlying value of $m_{\tilde{\nu}}=200 \mathrm{GeV}$. We also found that the results for $\rho_{\tilde{\chi}_{1}^{0}}$ and $m_{\tilde{\nu}}$ were not altered substantially when significantly worse experimental conditions were assumed. In the Higgsino case study, stringent global bounds were not found for any of the combinations of SUSY parameters. However, a number of interesting correlations were found, making it possible, for example, to exclude the grand unification condition $M_{1} / M_{2}=0.5$ in the gaugino region. In the mixed region example, results similar to those for the Higgsino region were achieved, with the exception that it was once again possible to set a stringent global upper bound on $m_{\tilde{\nu}}$.

In this study, we have only crudely simulated chargino events and detector effects. Although we have shown that, at least in some cases, our results are not very sensitive to the exact experimental assumptions, detailed event simulations and detector modeling would sharpen our results. Other work that may improve the results obtained here includes a study of chargino production at threshold [39], where chargino decays are more easily analyzed, and investigations of other SUSY processes, notably neutralino production, which may provide useful constraints in some regions of SUSY parameter space.

In summary, our results imply that if charginos are discovered at LEP II, they will bring not only the first experimental evidence for SUSY, but will also significantly restrict SUSY parameter space and may provide bounds on SUSY parameters of relevance to cosmology, grand unified theories, and future sparticle searches.

\section{ACKNOWLEDGMENTS}

It is a pleasure to thank M. Peskin for valuable comments and suggestions throughout the course of this work. In addition, we are grateful to T. Barklow for many discussions on

experimental issues, and to Y. Kizukuri and N. Oshimo for their generosity in helping us 
correct an error in our calculation of chargino branching fractions. We also thank L. Dixon, G. Farrar, M. Felcini, H. E. Haber, and H. Murayama for helpful conversations.

\section{APPENDIX: UNCERTAINTY ANALYSIS}

In this appendix we collect the various formulae for calculating uncertainties needed in the preceding sections.

The chargino and LSP masses determine the maximum and minimum dijet energies, $E_{j j}^{\max }$ and $E_{j j}^{\min }$, and the maximum dijet mass, $m_{j j}^{\max }$. Thus, if two of these three endpoints are measured, one can determine $m_{\tilde{\chi}_{1}^{ \pm}}$and $m_{\tilde{\chi}_{1}^{0}}$.

In terms of $m_{j j}^{\max }$ and either one of the energy endpoints $E_{j j}^{0} \equiv E_{j j}^{\max }$ or $E_{j j}^{\min }$, the masses $m_{\tilde{\chi}_{1}^{ \pm}}$and $m_{\tilde{\chi}_{1}^{0}}$ are given by

$$
\begin{aligned}
& m_{\tilde{\chi}_{1}^{ \pm}}\left(m_{j j}^{\max }, E_{j j}^{0}\right)=\frac{m_{j j}^{\max }}{2} \frac{2 E_{j j}^{0} E_{b}+\left(m_{j j}^{\max }\right)^{2}+E_{j j}^{0} \sqrt{4 E_{b}^{2}-4 E_{j j}^{0} E_{b}-m^{2}}}{\left(E_{j j}^{0}\right)^{2}+\left(m_{j j}^{\max }\right)^{2}}, \\
& m_{\tilde{\chi}_{1}^{0}}\left(m_{j j}^{\max }, E_{j j}^{0}\right)=m_{\tilde{\chi}_{1}^{ \pm}}\left(m_{j j}^{\max }, E_{j j}^{0}\right)-m_{j j}^{\max }
\end{aligned}
$$

where $E_{b}$ is the beam energy. The uncertainties in the mass determinations are given simply by adding the endpoint uncertainties in quadrature:

$$
\Delta m_{\tilde{\chi}_{1}^{ \pm}}=\left[\left(\frac{\partial m_{\tilde{\chi}_{1}^{ \pm}}}{\partial m_{j j}^{\max }} \Delta m_{j j}^{\max }\right)^{2}+\left(\frac{\partial m_{\tilde{\chi}_{1}^{ \pm}}}{\partial E_{j j}^{0}} \Delta E_{j j}^{0}\right)^{2}\right]^{\frac{1}{2}}
$$

and similarly for $\Delta m_{\tilde{\chi}_{1}^{0}}$.

To calculate $\sigma_{\text {total }}$ and $B_{l}$, one must measure at least two partial cross sections. If one has measured the mixed and hadronic partial cross sections $\sigma_{\text {mixed }}$ and $\sigma_{\text {hadronic }}$, the total cross section and leptonic branching fraction are

$$
\begin{aligned}
\sigma_{\text {total }} & =\frac{\left(\sigma_{\text {mixed }}+2 \sigma_{\text {hadronic }}\right)^{2}}{4 \sigma_{\text {hadronic }}}, \\
B_{l} & =\frac{\sigma_{\text {mixed }}}{\sigma_{\text {mixed }}+2 \sigma_{\text {hadronic }}},
\end{aligned}
$$

and their fractional uncertainties are given by

$$
\begin{gathered}
\left(\frac{\Delta \sigma_{\text {total }}}{\sigma_{\text {total }}}\right)^{2}=4 B_{l}^{2}\left(\frac{\Delta \sigma_{\text {mixed }}}{\sigma_{\text {mixed }}}\right)^{2}+\left(1-2 B_{l}\right)^{2}\left(\frac{\Delta \sigma_{\text {hadronic }}}{\sigma_{\text {hadronic }}}\right)^{2}, \\
\left(\frac{\Delta B_{l}}{B_{l}}\right)^{2}=\left(1-B_{l}\right)^{2}\left[\left(\frac{\Delta \sigma_{\text {mixed }}}{\sigma_{\text {mixed }}}\right)^{2}+\left(\frac{\Delta \sigma_{\text {hadronic }}}{\sigma_{\text {hadronic }}}\right)^{2}\right] .
\end{gathered}
$$

If $\sigma_{\text {mixed }}$ and $\sigma_{\text {leptonic }}$ are measured instead,

$$
\begin{aligned}
\sigma_{\text {total }} & =\frac{\left(\sigma_{\text {mixed }}+2 \sigma_{\text {leptonic }}\right)^{2}}{4 \sigma_{\text {leptonic }}}, \\
B_{l} & =\frac{2 \sigma_{\text {leptonic }}}{2 \sigma_{\text {leptonic }}+\sigma_{\text {mixed }}}
\end{aligned}
$$


and

$$
\begin{aligned}
\left(\frac{\Delta \sigma_{\text {total }}}{\sigma_{\text {total }}}\right)^{2} & =4\left(1-B_{l}\right)^{2}\left(\frac{\Delta \sigma_{\text {mixed }}}{\sigma_{\text {mixed }}}\right)^{2}+\left(1-2 B_{l}\right)^{2}\left(\frac{\Delta \sigma_{\text {leptonic }}}{\sigma_{\text {leptonic }}}\right)^{2}, \\
\left(\frac{\Delta B_{l}}{B_{l}}\right)^{2} & =\left(1-B_{l}\right)^{2}\left[\left(\frac{\Delta \sigma_{\text {mixed }}}{\sigma_{\text {mixed }}}\right)^{2}+\left(\frac{\Delta \sigma_{\text {leptonic }}}{\sigma_{\text {leptonic }}}\right)^{2}\right] .
\end{aligned}
$$

If all three partial cross sections are measured, they may all be used to improve the determinations of $\sigma_{\text {total }}$ and $B_{l}$. In this study, the $Y$ mode partial cross section is measured instead of the mixed mode. After lepton universality is verified, $\sigma_{\text {mixed }}=\frac{5}{4} \sigma_{Y}$, and one can simply replace $\sigma_{\text {mixed }}$ by $\sigma_{Y}$ in the above formulae.

Finally, we must calculate the uncertainty of $A_{F B}^{j j}$. The forward-backward asymmetry is $A_{F B}^{j j}=2 p-1$, where $p$ is the fraction of events in the forward hemisphere, and the standard error of an estimate of a population proportion is

$$
\sigma(p)=\sqrt{\frac{p(1-p)}{N}},
$$

where $N$ is the number of samples. The $1 \sigma$ uncertainty in the measurement of $A_{F B}^{j j}$ is therefore given by

$$
\sigma\left(A_{F B}^{j j}\right)=2 \sigma(p)=\sqrt{\frac{1-\left(A_{F B}^{j j}\right)^{2}}{N}} .
$$




\section{REFERENCES}

[1] For reviews of supersymmetry and the minimal supersymmetric standard model, see H.E. Haber and G.L. Kane, Phys. Rep. 117, 75 (1985); H.P. Nilles, Phys. Rep. 110, 1 (1984); P. Nath, R. Arnowitt, and A.H. Chamseddine, Applied $N=1$ Supergravity, ICTP Series in Theoretical Physics, Vol. 1 (World Scientific, Singapore, 1984); X. Tata, in The Standard Model and Beyond, proceedings of the Ninth Symposium on Theoretical Physics, Mt. Sorak, Korea, 1990, edited by J.E. Kim (World Scientific, River Edge, New Jersey, 1991), p. 304 .

[2] D.A. Dicus, S. Nandi, W. Repko, and X. Tata, Phys. Rev. Lett. 51, 1030 (1983); V. Barger, R. W. Robinett, W. Y. Keung, and R. J. N. Phillips, Phys. Lett. 131B, 372 (1983); J. M. Frère and G. L. Kane, Nucl. Phys. B223, 331 (1983); G. Altarelli, B. Mele, and S. Petrarca, Nucl. Phys. B245, 215 (1984); S. Dawson, E. Eichten, and C. Quigg, Phys. Rev. D 31, 1581 (1985); T. Schimert, C. Burgess, and X. Tata, Phys. Rev. D 32, 707 (1985); P. Chiappetta et al., Nucl. Phys. B262, 495 (1985); H. Baer, K. Hagiwara, and X. Tata, Phys. Rev. D 35, 1598 (1987); X. Tata and D. A. Dicus, Phys. Rev. D 35, 2110 (1987); J. Gunion, H. Haber, R. M. Barnett, M. Drees, D. Karatas, X. Tata, and H. Baer, Int. J. Mod. Phys. A2, 1145 (1987); R. Arnowitt and P. Nath, Phys. Rev. D 35, 1085 (1987); B. F. L. Ward, Phys. Rev. D 35, 2092 (1987); T. Fukai, Y. Kizukuri, N. Oshimo, Y. Otake, and N. Sugiyama, Prog. Theor. Phys. 78, 395 (1987); J. Gunion and H. Haber, Phys. Rev. D 37, 2515 (1988); H. Baer, A. Bartl, D. Karatas, W. Majerotto, and X. Tata, Int. J. Mod. Phys. A4, 4111 (1989); H. Baer, V. Barger, R. J. N. Phillips, and X. Tata, Phys. Lett. 220B, 303 (1989); Y. Kizukuri and N. Oshimo, Phys. Lett. 220B, 293 (1989); R. Barbieri, F. Caravaglios, M. Frigeni, and M. L. Mangano, Nucl. Phys. B367, 28 (1991); M. H. Nous, M. El-Kishen, and T. A. El-Azem, Mod. Phys. Lett. A7, 1535 (1992); H. N. Saif and C. S. Kalman, Z. Phys. C 56, 447 (1992); H. Baer, C. Kao, and X. Tata, Phys. Rev. D 48, 5175 (1993).

[3] P. Chiappetta, F. M. Renard, J. Soffer, P. Sorba, and P. Taxil, Nucl. Phys. B262, 495 (1985); B279, 824(E) (1987).

[4] A. Bartl, H. Fraas, and W. Majerotto, Z. Phys. C 30, 441 (1986).

[5] A. Bartl, H. Fraas, W. Majerotto, and B. Mösslacher, Z. Phys. C 55, 257 (1992).

[6] C. Dionisi et al., in Proceedings of the ECFA Workshop on LEP 200, Aachen, Federal Republic of Germany, 29 September - 1 October 1986, edited by A. Böhm and W. Hoogland, Vol. II, CERN Report No. 87-08, June 1987, p. 380.

[7] M. Chen, C. Dionisi, M. Martinez, and X. Tata, Phys. Rept. 159, 201 (1988).

[8] J.-F. Grivaz, in Proceedings of INFN Eloisatron Project Workshop, Twenty-third: The Decay Properties of SUSY Particles, Erice, Italy, September 28 - October 4, 1992, edited by L. Cifarelli and V. A. Khoze (World Scientific, River Edge, New Jersey, 1993).

[9] C. Ahn et al., SLAC Report No. 329, 1988 (unpublished).

[10] J.-F. Grivaz, in Proceedings of the Workshop on Physics and Experiments with Linear Colliders, Saariselka, Finland, 1991, Vol. 1, edited by R. Orava, P. Eerola, and M. Nordberg (World Scientific, River Edge, New Jersey, 1992), p. 353.

[11] Particle Data Group, L. Montanet et al., Phys. Rev. D 50, 1173 (1994).

[12] ALEPH Collaboration, D. Décamp et al., Phys. Rep. 216, 253 (1992).

[13] D. Treille, CERN Report No. CERN-PPE-54-REV, 1993 (unpublished). 
[14] The SLD Collaboration, K. Abe et al., SLAC Report No. SLAC-PUB-6456, 1994 (unpublished).

[15] G. Altarelli, R. Barbieri, and F. Caravaglios, Phys. Lett. 314B, 357 (1993).

[16] The LEP Collaborations ALEPH, DELPHI, L3, OPAL, and The LEP Electroweak Working Group, CERN Report No. CERN-PPE-93-157, 1993 (unpublished).

[17] The SLD Collaboration, K. Abe et al., SLAC Report No. SLAC-PUB-6569, 1994 (unpublished).

[18] M. Boulware and D. Finnell, Phys. Rev. D 44, 2054 (1991).

[19] J.D. Wells, C. Kolda, and G.L. Kane, Report No. UM-TH-94-23, 1994 (unpublished).

[20] A. Bartl, H. Fraas, and W. Majerotto, Nucl. Phys. B278, 1 (1986); A. Bartl, W. Majerotto, and B. Mösslacher, in Phenomenological Aspects of Supersymmetry, proceedings of a series of seminars held at the Max-Planck-Institut für Physik, Munich, Germany, 1991, edited by W. Hollik, R. Rückl, and J. Wess (Springer-Verlag, Berlin, 1993), p. 119.

[21] JLC Group, JLC-I, Tsukuba, Japan, 1992 (KEK Report 92-16, Tsukuba, 1992).

[22] T. Tsukamoto, K. Fujii, H. Murayama, M. Yamaguchi, and Y. Okada, KEK Report No. 93-146, 1993 (unpublished).

[23] S. Orito, in Proceedings of the Workshop on Physics and Experiments with Linear $e^{+} e^{-}$ Colliders, Waikoloa, Hawaii, 1993, edited by F.A. Harris, S.L. Olsen, S. Pakvasa, and X. Tata (World Scientific, Singapore, 1993), p. 230.

[24] R. Becker and C. Vander Velde, in Proceedings of the Workshop on Physics and Experiments with Linear $e^{+} e^{-}$Colliders [23], p. 808.

[25] T. Tsukamoto, in Proceedings of the Workshop on Physics and Experiments with Linear $e^{+} e^{-}$Colliders [23], p. 814.

[26] T. Kon, in Proceedings of the Workshop on Physics and Experiments with Linear $e^{+} e^{-}$ Colliders [23], p. 822.

[27] J. Feng and D. Finnell, Phys. Rev. D 49, 2369 (1994).

[28] A. Leike, Int. J. Mod. Phys. A4, 845 (1989).

[29] A. Leike, Int. J. Mod. Phys. A3, 2895 (1988).

[30] K. Olive and M. Srednicki, Phys. Lett. 230B, 78 (1989); L. Roszkowski, Phys. Lett. 262B, 59 (1991); K. Griest and D. Seckel, Phys. Rev. D 43, 3191 (1991); S. Mizuta and M. Yamaguchi, Phys. Lett. 298B, 120 (1993); M. Drees and M. Nojiri, Phys. Rev. D 47, 376 (1993).

[31] J.F. Gunion and H.E. Haber, Nucl. Phys. B272, 1 (1986); B402, 567(E) (1993).

[32] L. Girardello and M.T. Grisaru, Nucl. Phys. B194, 65 (1982).

[33] J. Ellis and D.V. Nanopoulos, Phys. Lett. 110B, 44 (1982); T. Inami and C.S. Lim, Nucl. Phys. B207, 533 (1982); M.J. Duncan and J. Trampetic, Phys. Lett. 134B, 439 (1984); J. Hagelin, S. Kelley, and T. Tanaka, Nucl. Phys. B415, 293 (1994).

[34] D. Pierce and A. Papadopoulos, Report Nos. JHU-TIPAC-930030, PURD-TH-93-13, 1993 (unpublished); Report No. JHU-TIPAC-940001, 1994 (unpublished).

[35] A.B.Lahanas, K. Tamvakis, and N. D. Tracas, Phys. Lett. 324B, 387 (1994).

[36] K. Inoue, A. Kakuto, H. Komatsu, and S. Takeshita, Prog. Theor. Phys. 68, 927 (1982); L. Alvarez-Gaume, J. Polchinski, and M. Wise, Nucl. Phys. B221, 495 (1983).

[37] J.F. Gunion and H.E. Haber, Phys. Rev. D 37, 2515 (1988).

[38] CDF Collaboration, F. Abe et al., Fermilab Report No. FERMILAB-PUB-94-116-E, 1994 (unpublished); Fermilab Report No. FERMILAB-PUB-94-097-E, 1994 (unpublished). 
[39] J. Feng and M. Strassler (in preparation).

[40] N. Oshimo and Y. Kizukuri, Phys. Lett. 186B, 217 (1987).

[41] K. Hagiwara and D. Zeppenfeld, Nucl. Phys. B274, 1 (1986).

[42] J. H. Friedman, J. Comput. Phys., 7, 201 (1971).

[43] H. Murayama, I. Watanabe, and K. Hagiwara, KEK Report No. 91-11, 1992 (unpublished).

[44] A. Miyamoto, in Proceedings of the Second Workshop on Japan Linear Collider (JLC), KEK, November 6-8, 1990, edited by S. Kawabata, KEK Proceedings 91-10, November 1991, p. 256.

[45] O. Adriani et al., Nucl. Instrum. Meth. A302, 53 (1991); L3 Collaboration, O. Adriani et al., Phys. Rep. 236, 1 (1993).

[46] M. Felcini, private communication. 


\section{FIGURES}

FIG. 1. The three chargino production diagrams for $e^{+} e^{-}$collisions.

FIG. 2. The three-body chargino decay diagrams. There exist separate scalar partners for each chirality of fermion, and so there are a total of six hadronic and five leptonic decay diagrams.

FIG. 3. Contours of constant $m_{\tilde{\chi}_{1}^{ \pm}}($in $\mathrm{GeV})$ for $\tan \beta=4$. The cross-hatched region is excluded by the experimental bounds $m_{\tilde{\chi}_{1}^{ \pm}}>45 \mathrm{GeV}$ and $m_{\tilde{\chi}_{1}^{0}}>20 \mathrm{GeV}$. In the hatched regions, $m_{\tilde{\chi}_{1}^{ \pm}}>95 \mathrm{GeV}$, so charginos are kinematically inaccessible.

FIG. 4. Contours of constant $m_{\tilde{\chi}_{1}^{0}}$ (in $\mathrm{GeV}$ ) for fixed $\tan \beta=4$ and three values of the gaugino mass ratio $M_{1} / M_{2}$ : (a) 0.5 , (b) 0.75 , and (c) -0.5 . The hatched and cross-hatched regions are as in Fig. 3 .

FIG. 5. Contours of constant $\rho_{\tilde{\chi}_{1}^{ \pm}}$for $\tan \beta=4$, with hatched and cross-hatched regions as in Fig. 3. As defined in the text, $0 \leq \rho_{\tilde{\chi}_{1}^{ \pm}} \leq 0.2$ in the Higgsino region, $0.2<\rho_{\tilde{\chi}_{1}^{ \pm}}<0.9$ in the mixed region, and $0.9 \leq \rho_{\tilde{\chi}_{1}^{ \pm}} \leq 1.0$ in the gaugino region. The gaugino content approaches one asymptotically as $|\mu| \rightarrow \infty$ in the gaugino region and zero asymptotically as $M_{2} \rightarrow \infty$ in the Higgsino region.

FIG. 6. Contours of constant $E^{\max }$ (in $\mathrm{GeV}$ ), the maximum jet or lepton energy for decay products from chargino events. The plot is for $M_{1} / M_{2}=0.5$ and $\tan \beta=4$. The hatched and cross-hatched regions are as in Fig. 3 , and the vertical and horizontal scales are chosen to emphasize the Higgsino region. We see that in the Higgsino region, for points in parameter space with large $M_{2}$ or near threshold, the decay products may be too soft to be useful for precision measurements.

FIG. 7. The total cross section $\sigma_{\text {total }}$ before cuts (solid curve) as a function of $\sqrt{s}$ for parameters $\left(\mu, M_{2}, \tan \beta, m_{\tilde{\nu}}\right)=(-400,75,4,200)$ in the gaugino region. For comparison, a unit of $\mathrm{R}$ is also plotted (dashed curve). We note the sudden rise in cross section, characteristic of fermion production.

FIG. 8. Contours of constant value of the ratio $A_{\text {central }}^{\tilde{\chi}^{ \pm}} / A_{\text {central }}^{(0) \tilde{\chi}^{ \pm}}$, defined in the text. The hatched and cross-hatched regions are as in Fig. 3. The contours are plotted in the $\left(\mu, M_{2}\right)$ plane for fixed $\tan \beta=4$ and (a) $m_{\tilde{\nu}}=200 \mathrm{GeV}$ and (b) $m_{\tilde{\nu}}=100 \mathrm{GeV}$ and are chosen to emphasize the range of the ratio. In the case $m_{\tilde{\nu}}=200 \mathrm{GeV}$, where the perturbative analysis is expected to hold, we see that the ratio is approximately one, as expected. For $m_{\tilde{\nu}}=100 \mathrm{GeV}$, beyond the range in which the perturbative analysis can be expected to be valid, we see that this behavior nevertheless persists, and deviations are small. 
FIG. 9. Contours of constant $\sigma_{\text {total }}$ in picobarns in the $\left(\mu, M_{2}\right)$ plane for fixed $\tan \beta=4$ and (a) $m_{\tilde{\nu}}=1 \mathrm{TeV}$ and (b) $m_{\tilde{\nu}}=150 \mathrm{GeV}$. The hatched and cross-hatched regions are as in Fig. 3 . The cross section rises quickly near threshold. The lower cross sections in (b) are a result of the large destructive interference of the sneutrino diagram.

FIG. 10. Cross sections as functions of $m_{\tilde{\nu}}$ are plotted for $\tan \beta=4$ and four representative $\left(\mu, M_{2}\right)$ points: $(-400,75)$ in the gaugino region (solid), $(-90,115)$ in the mixed region (dashed), $(-75,250)$ in the Higgsino region (dot-dashed), and $(-78,1000)$ in the far Higgsino region (dotted). The $\tilde{\nu}$ diagram gives a large and destructive contribution for the gaugino and mixed points. As the Higgsino component increases, the dependence of the cross section on $m_{\tilde{\nu}}$ decreases.

FIG. 11. Contours of constant $A_{F B}^{\tilde{\chi}^{ \pm}}$in percent for $\tan \beta=4$ and (a) $m_{\tilde{\nu}}=1 \mathrm{TeV}$ and (b) $m_{\tilde{\nu}}=150 \mathrm{GeV}$. The hatched and cross-hatched regions are as in Fig. 3. In the large $m_{\tilde{\nu}}$ limit, $A_{F B}^{\tilde{\chi}^{ \pm}} \rightarrow 0$ in both the gaugino and Higgsino limits, with only the mixed region giving a significant negative $A_{F B}^{\tilde{\chi}^{ \pm}}$. For low $m_{\tilde{\nu}}$, the $t$-channel sneutrino exchange diagram can lead to large and positive $A_{F B}^{\tilde{\chi}^{ \pm}}$.

FIG. 12. Contours of constant $\sigma_{R} / \sigma_{L}$ in percent in the $\left(\mu, M_{2}\right)$ plane for $\tan \beta=4$ and $m_{\tilde{\nu}}=150 \mathrm{GeV}$. The ratio never rises above $15 \%$ in the allowed bands, and is approximately $2 \%$ in much of the gaugino region. The hatched and cross-hatched regions are as in Fig. 3 .

FIG. 13. Contours of constant value of the leptonic branching fraction $B_{l}$ in the $\left(\mu, M_{2}\right)$ plane for $M_{1} / M_{2}=0.5$ and three sets of parameters $\left(\tan \beta, m_{\tilde{l}}, m_{\tilde{q}}\right):$ (a) $(2,200,200)$, (b) $(2,200,800)$, and (c) $(10,200,200)$. The gaugino region has been magnified, and the hatched and cross-hatched regions are as in Fig. 3. For all figures, the value of $B_{l}$ is $\frac{1}{3}$ in the Higgsino region and grows as one approaches the gaugino region. The growth is faster for large $\tan \beta$ (c) than for low $\tan \beta$ (a). In (a) and (b) the $B_{l}$ contours differ by approximately $5 \%$ in the far gaugino region, consistent with Eq. (55). Note also the "pocket" in the $\mu<0$ near gaugino region, where $B_{l}<\frac{1}{3}$.

FIG. 14. The $m_{\tilde{\chi}_{1}^{ \pm}}$measurement restricts the $\left(\mu, M_{2}, \tan \beta\right)$ space to two thin sheets $\mathcal{S}$, which are then flattened into the plane $\mathcal{T}$ with the transformation $\left(\mu, M_{2}, \tan \beta\right) \rightarrow(\alpha, \tan \beta)$, where $\alpha=\arctan \left(M_{2} / \mu\right)$. This transformation is illustrated schematically here. For large $\tan \beta$, observables are symmetric under $\mu \leftrightarrow-\mu$, that is, under $\alpha \leftrightarrow 180^{\circ}-\alpha$.

FIG. 15. The dijet (a) mass spectrum and (b) energy spectrum, after cuts, for the gaugino case $\left(\mu, M_{2}, \tan \beta, M_{1} / M_{2}, m_{\tilde{l}}, m_{\tilde{q}}\right)=(-400,75,4,0.5,200,300)$ with integrated luminosity $1 \mathrm{fb}^{-1}$. In these distributions, hadrons from $\tau$ lepton decays have not been included. We see that finite detector resolution effects cause the spectra to have tails that exceed the theoretical limits, but despite this, the endpoints are fairly sharp. We estimate that the $1 \sigma$ uncertainty of $m_{j j}^{\max }$ is $2 \mathrm{GeV}$, and that for $E_{j j}^{\max }$ is $3 \mathrm{GeV}$. Note that very few events have dijets with low invariant mass, and it is therefore possible to distinguish hadrons that result from $\tau$ decays and those that result from hadronic chargino decays. 
FIG. 16. Gaugino example contours for the (a) minimum and (b) maximum values of $M_{1} / M_{2}$ in the $(\alpha, \tan \beta)$ plane, as defined in Sec. $\mathrm{VA}$. The cross-hatched area in the $\alpha>90^{\circ}$ mixed region with $\tan \beta \approx 1$ is excluded by the $m_{\tilde{\chi}_{1}^{0}}$ measurement. The approximate symmetry $\alpha \leftrightarrow 180^{\circ}-\alpha$ $(\mu \leftrightarrow-\mu)$ for large $\tan \beta$ is already in evidence at $\tan \beta=10$.

FIG. 17. Gaugino example contours for the (a) minimum and (b) maximum values of $m_{\tilde{l}}$ in the $(\alpha, \tan \beta)$ plane, as defined in Sec. $\mathrm{VA}$. The cross-hatched area in the Higgsino region is excluded because the measured $\sigma_{\text {total }}$ is too large to be compatible with any $m_{\tilde{l}}$.

FIG. 18. Gaugino example contours for the (a) minimum and (b) maximum values of $m_{\tilde{q}}$ in the $(\alpha, \tan \beta)$ plane, as defined in Sec. $\mathrm{VA}$. The $\alpha>90^{\circ}(\mu<0)$ gaugino region has been magnified. (Similar features are seen in the $\alpha<90^{\circ}(\mu>0)$ gaugino region.) The hatched and cross-hatched regions are excluded by the $B_{l}$ measurement: in the hatched region $B_{l}$ is too large, and in the cross-hatched region $B_{l}$ is too small.

FIG. 19. The allowed region in the $(\alpha, \tan \beta)$ plane for the gaugino case study with both signs of $M_{1}$ allowed. The hatched regions are excluded by the measurements of $m_{\tilde{\chi}_{1}^{ \pm}}, m_{\tilde{\chi}_{1}^{0}}, \sigma_{\text {total }}$, and $B_{l}$, which confine the allowed region to narrow strips in the gaugino region. The dot indicates the underlying value of $(\alpha, \tan \beta)$ for the gaugino case study.

FIG. 20. Plot of $A_{F B}^{j j}$ vs. $A_{F B}^{\tilde{\chi}^{ \pm}}$for several points in the allowed region of the gaugino case study. The solid line is the measured $A_{F B}^{j j}$ from the Monte Carlo simulation, and the dashed lines give the $1 \sigma$ uncertainties in this measurement. Points with $\mu<0$ are given by filled circles, and all points with $\mu>0$ (open circles) are seen to be excluded by the $A_{F B}^{j j}$ measurement. The case study is indicated by a star.

FIG. 21. The dijet (a) mass spectrum and (b) energy spectrum, after cuts, for the Higgsino case $\left(\mu, M_{2}, \tan \beta, M_{1} / M_{2}, m_{\tilde{l}}, m_{\tilde{q}}\right)=(-75,250,4,0.5,200,300)$ with integrated luminosity $1 \mathrm{fb}^{-1}$. We estimate that the $1 \sigma$ uncertainty of $m_{j j}^{\max }$ is $1 \mathrm{GeV}$, and that for $E_{j j}^{\max }$ is $2 \mathrm{GeV}$.

FIG. 22. Higgsino example contours for the (a) minimum and (b) maximum values of $M_{1} / M_{2}$. In (b), two $M_{1} / M_{2}=0.8$ contours have been removed from the Higgsino region for clarity. The cross-hatched area in the $\alpha>90^{\circ}$ mixed region with $\tan \beta \approx 1$ is excluded by the $m_{\tilde{\chi}_{1}^{0}}$ measurement alone.

FIG. 23. Higgsino example contours for the (a) minimum and (b) maximum values of $m_{\tilde{l}}$. Stringent bounds are found in the gaugino region, but no limits are obtained in the Higgsino region. 
FIG. 24. Higgsino example contours for the (a) minimum and (b) maximum values of $m_{\tilde{q}}$. Two areas in the $\alpha>90^{\circ}(\mu<0)$ gaugino region has been magnified. The cross-hatched region of (a) and the hatched region of (b) have $B_{l}$ values that are too low and too high, respectively, and are excluded.

FIG. 25. The allowed region in the $(\alpha, \tan \beta)$ plane for the Higgsino case study, with both signs of $M_{1}$ allowed. The cross-hatched regions are excluded by the measurements of $m_{\tilde{\chi}_{1}^{ \pm}}, m_{\tilde{\chi}_{1}^{0}}, \sigma_{\text {total }}$, and $B_{l}$. The dot indicates the value of $(\alpha, \tan \beta)$ for the case study.

FIG. 26. Plot of $A_{F B}^{j j}$ vs. $A_{F B}^{\tilde{\chi}^{ \pm}}$for several points in the allowed region of the Higgsino case study. The solid line is the measured $A_{F B}^{j j}$ from the Monte Carlo simulation, and the dashed lines give the $1 \sigma$ uncertainties in this measurement. The case study is indicated by a star.

FIG. 27. Mixed example contours for the (a) minimum and (b) maximum values of $M_{1} / M_{2}$. The cross-hatched area in the mixed region with $\tan \beta \approx 1$ is excluded by the $m_{\tilde{\chi}_{1}^{0}}$ measurement alone.

FIG. 28. Mixed example contours for the (a) minimum and (b) maximum values of $m_{\tilde{l}}$. The cross-hatched area in the Higgsino region has $\sigma_{\text {total }}$ values that are higher than that measured and is excluded. In this case, an upper bound on $m_{\tilde{l}}$ may be obtained from the $\sigma_{\text {total }}$ measurement alone.

FIG. 29. The allowed region in the $(\alpha, \tan \beta)$ plane for the mixed region case study with both signs of $M_{1}$ allowed. The cross-hatched regions are excluded by the measurements of $m_{\tilde{\chi}_{1}^{ \pm}}, m_{\tilde{\chi}_{1}^{0}}$, $\sigma_{\text {total }}$, and $B_{l}$, and the dot indicates the value of $(\alpha, \tan \beta)$ for the case study.

FIG. 30. Plot of $A_{F B}^{j j}$ vs. $A_{F B}^{\tilde{\chi}^{ \pm}}$for several points in the allowed region of the mixed region case study. The solid line is the measured $A_{F B}^{j j}$ from the Monte Carlo simulation, and the dashed lines give the $1 \sigma$ uncertainties in this measurement. The specific parameters chosen for the case study lie at an extreme value of $A_{F B}^{j j}$ and indicate that this measurement may be able to reduce the allowed region substantially. The case study is indicated by a star. 
This figure "fig1-1.png" is available in "png" format from: http://arxiv.org/ps/hep-ph/9408359v1 
This figure "fig1-2.png" is available in "png" format from: http://arxiv.org/ps/hep-ph/9408359v1 
This figure "fig1-3.png" is available in "png" format from: http://arxiv.org/ps/hep-ph/9408359v1 
This figure "fig1-4.png" is available in "png" format from: http://arxiv.org/ps/hep-ph/9408359v1 
This figure "fig1-5.png" is available in "png" format from: http://arxiv.org/ps/hep-ph/9408359v1 
This figure "fig1-6.png" is available in "png" format from: http://arxiv.org/ps/hep-ph/9408359v1 
This figure "fig1-7.png" is available in "png" format from: http://arxiv.org/ps/hep-ph/9408359v1 
This figure "fig1-8.png" is available in "png" format from: http://arxiv.org/ps/hep-ph/9408359v1 
This figure "fig1-9.png" is available in "png" format from: http://arxiv.org/ps/hep-ph/9408359v1 
This figure "fig1-10.png" is available in "png" format from: http://arxiv.org/ps/hep-ph/9408359v1 
This figure "fig1-11.png" is available in "png" format from: http://arxiv.org/ps/hep-ph/9408359v1 
This figure "fig1-12.png" is available in "png" format from: http://arxiv.org/ps/hep-ph/9408359v1 
This figure "fig1-13.png" is available in "png" format from: http://arxiv.org/ps/hep-ph/9408359v1 
This figure "fig1-14.png" is available in "png" format from: http://arxiv.org/ps/hep-ph/9408359v1 
This figure "fig1-15.png" is available in "png" format from: http://arxiv.org/ps/hep-ph/9408359v1 